Supporting Information

for

\title{
Light Directs Monomer Coordination in Catalyst-Free Grignard Photopolymerization
}

Eliot F. Woods, Alexandra J. Berl, Leanna P. Kantt, Christopher T. Eckdahl, Michael R. Wasielewski, Brandon E. Haines* and Julia A. Kalow*

Department of Chemistry, Northwestern University, 2145 Sheridan Rd, Evanston, IL 60208

Department of Chemistry, Westmont College, 955 La Paz Road, Santa Barbara, CA 93108

E-mail: bhaines@westmont.edu, jkalow@northwestern.edu

\section{Table of Contents}

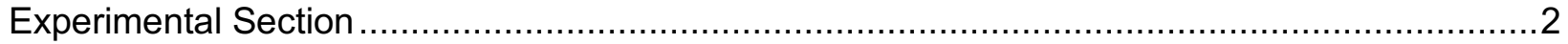

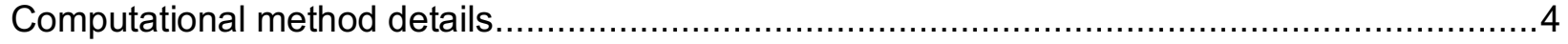

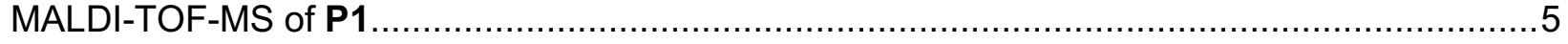

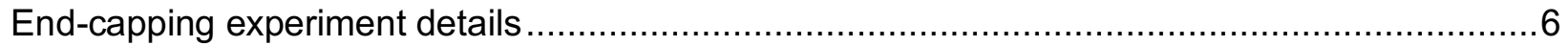

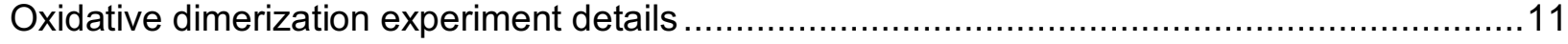

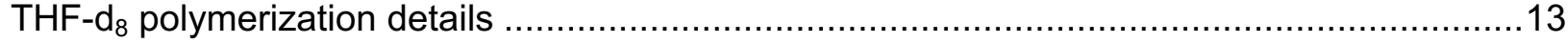

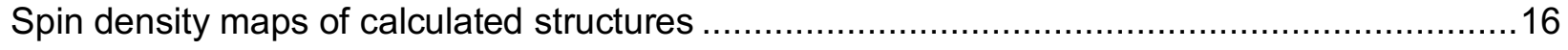

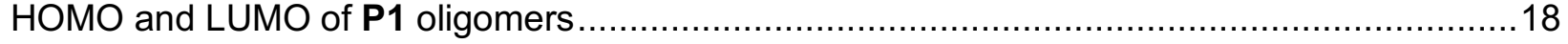

Additional details for monomer-coordinated hexamer structures ......................................20

Excited-state geometry for monomer-coordinated structures ….......................................25

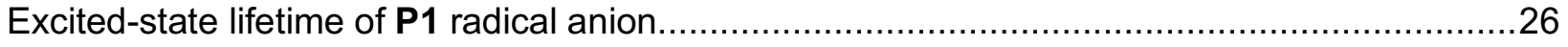

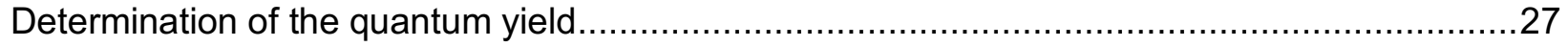

Calculations for aggregation of benzotriazole Grignard monomer .....................................30

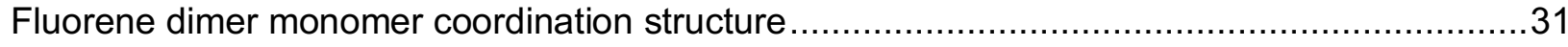

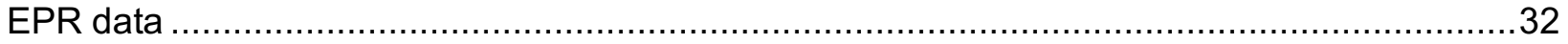

Cartesian coordinates of calculated structures with energies and frequency analysis .............35

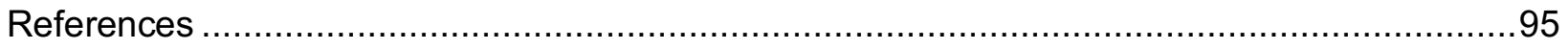




\section{Experimental Section}

General procedures. Unless otherwise noted, reactions were performed under Ar atmosphere in oven-dried $\left(150^{\circ} \mathrm{C}\right)$ glassware. Reaction progress was monitored by thin layer chromatography (EMD $250 \mu \mathrm{m}$ silica gel 60-F254 plates) and visualized with potassium permanganate stain, or ceric ammonium molybdate stain and heat. Automated column chromatography was performed using SiliCycle SiliaFlash F60 (40-63 $\mathrm{m}, 60 \AA)$ in SNAP cartridges on a Biotage Isolera One. Organic solvents were removed in vacuo using a rotary evaporator (Büchi Rotovapor R-100, 20200 torr) and residual solvent was removed under high vacuum (<0.1 torr).

Materials. Commercial reagents were purchased from Sigma-Aldrich, Acros, Alfa Aesar, TCl, or Oakwood and used as received unless otherwise noted. 4,7-dibromo-2-(2-octyldodecyl)-2Hbenzo[d][1,2,3]triazole was synthesized according to literature procedures. ${ }^{1}$ 2,6difluorobenzaldehyde was distilled over calcium hydride and stored under nitrogen before use. All solvents were purified and dried using a solvent-purification system that contained activated alumina. Additionally, THF was degassed before being stored in a nitrogen-filled glove box over activated $3 \AA \AA$ sieves. THF-d8 was distilled over calcium hydride before being degassed and stored in a nitrogen-filled glove box over activated $3 \AA \AA$ sieves.

Instrumentation. Proton nuclear magnetic resonance ( ${ }^{1} \mathrm{H}$ NMR) spectra and carbon nuclear magnetic resonance $\left({ }^{13} \mathrm{C}\right.$ NMR) spectra were recorded on Bruker AVANCE-500 spectrometers at $500 \mathrm{MHz}, 600 \mathrm{MHz}$, and $125 \mathrm{MHz}$, and referenced to the solvent residual peaks. NMR data are represented as follows: chemical shift $(\delta \mathrm{ppm})$, multiplicity $(\mathrm{s}=$ singlet, $\mathrm{d}=$ doublet, $\mathrm{t}=$ triplet, $\mathrm{q}=$ quartet, $\mathrm{m}=$ multiplet), coupling constant in Hertz $(\mathrm{Hz})$, integration. Size exclusion chromatography (SEC) measurements were performed in stabilized, HPLC-grade tetrahydrofuran using an Agilent 1260 Infinity II system with variable-wavelength diode array (254, 400, 480, 530, and $890 \mathrm{~nm}$ ) and refractive index detectors, guard column (Agilent PLgel; $5 \mu \mathrm{m} ; 50 \times 7.5 \mathrm{~mm}$ ), and three analytical columns (Agilent PLgel; $5 \mu \mathrm{m} ; 300 \times 7.5 \mathrm{~mm} ; 105,104$, and $103 \AA$ pore sizes). The instrument was calibrated with narrow dispersity polystyrene standards between $640 \mathrm{~g} / \mathrm{mol}$ and $2300 \mathrm{~kg} / \mathrm{mol}$ (Polymer Standards Service $\mathrm{GmbH}$ ). All runs were performed at $1.0 \mathrm{~mL} / \mathrm{min}$ flow rate and $40^{\circ} \mathrm{C}$. Molecular weight values are calculated based on the refractive index signal. White $(4000 \mathrm{~K})$ LEDs (power $=16.2 \mathrm{~W})$ were purchased from superbrightleds.com (see figure S1 for emission spectra of LEDs). Emission spectra of LEDs was measured with an Ocean Optics Fiber coupled CCD spectrometer model number ADC1000-USB. Matrix-assisted laser desorption/ionization time of flight mass spectrometry (MALDI-TOF-MS) was performed on Bruker Rapiflex MALDI-TOF Tissuetyper in linear positive mode. Samples were prepared by dissolving $0.5 \mu \mathrm{L}$ of a $2 \mathrm{~g} / \mathrm{mL}$ solution of sample in THF with 10-100 $\mu \mathrm{L}$ 1,1,3,3-tetraphenylbutadiene (25 $\mathrm{mg} / \mathrm{mL}$ in THF). The sample:matrix ratios were varied to achieve good signal to noise. The glove box in which specified procedures were carried out was an MBraun Unilab Pro with $\mathrm{N}_{2}$ atmosphere. Continuous wave electron paramagnetic resonance (CW-EPR) experiments were performed at room temperature. The samples were in quartz tubes with $2.4 \mathrm{~mm}$ o.d. and $2 \mathrm{~mm}$ i.d., prepared in a nitrogen filled glovebox and sealed with UV-cure epoxy (IllumaBond 607180RCL16). These experiments were performed at X-band on a Bruker ESP300E spectrometer equipped with a Bruker SQHE resonator at room temperature at a frequency of $9.56 \mathrm{GHz}$. These spectra were measured with $0.1 \mathrm{mT}$ magnetic field modulation and $100 \mathrm{kHz}$ modulation frequency. The microwave power was $4.8 \mathrm{~mW}(16 \mathrm{~dB})$. 


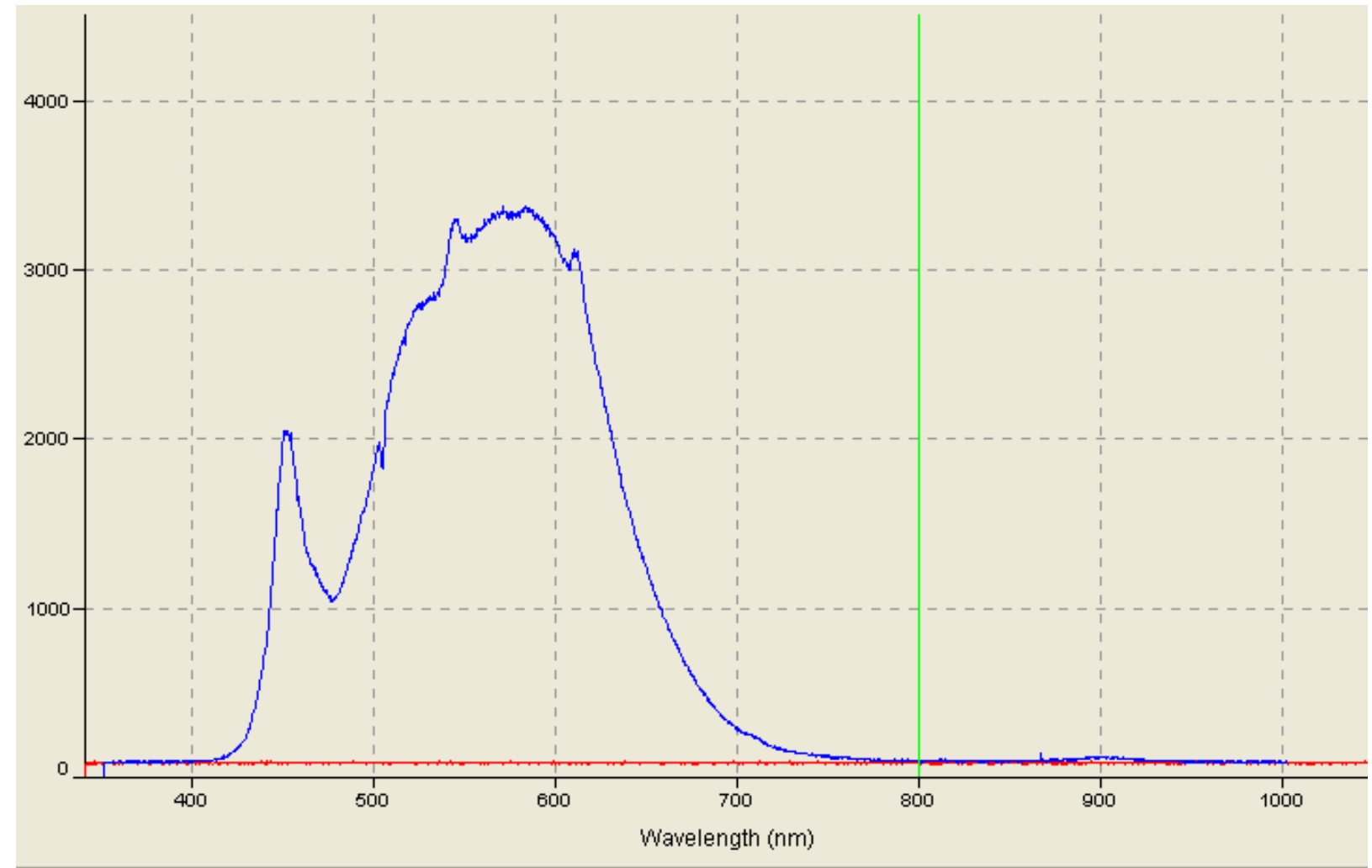

Figure S1. Measured emission spectra of "4000 K" white LEDs used. 


\section{Computational method details}

All calculations were carried out with the ORCA 4.2.1 program. ${ }^{2,3}$ Avogadro was used as a molecular editor and viewer. ${ }^{4}$ Full geometry optimizations of stationary points were calculated at the CAM-B3LYP/6-311G(d)/CPCM(THF) level of theory. ${ }^{5,6}$ Energies reported for stationary points are Gibbs free energies calculated at $298 \mathrm{~K}$ and $1.0 \mathrm{M}$ standard state. Frequency calculations were preformed to characterize the structures either as a minimum (zero negative frequencies) or as a transition state (one negative frequency). All frequency calculations performed applied the quasi-rigid rotor harmonic oscillator approximation ${ }^{7}$ to frequencies below $35 \mathrm{~cm}^{-1}$. Intrinsic reaction coordinate (IRC) calculations were performed for transition states to ensure they connect the correct reactant and product. Excited-state geometry optimizations were performed in the gasphase at the CAM-B3LYP/6-311G(d) level of theory, and their reported Gibbs free energies corrected with electronic energies from single point energy calculations using the CPCM(THF) implicit solvent model.

Note on the choice of the 6-311G(d) basis set. While it is common practice to use diffuse basis sets when modeling anions, we found serious and unresolvable SCF convergence issues when attempting to use common diffuse basis sets such as 6-311+G(d), ma-def2-TZVP, and aug-ccpVTz. Previous studies have shown that anions in polyaromatic hydrocarbons with extended conjugation frequently encounter SCF convergence problems when modeling with diffuse basis sets. ${ }^{8}$ The calculated geometries and energies of anions with extended conjugation are largely insensitive to modeling with non-diffuse basis sets when compared to results from diffuse basis sets.

Using a single brominated unit of BTz as a model system, we were able to converge the SCF of the radical anion using a complete rebuild of the Fock matrix during SCF iterations. We find the difference of the single-point energy between the diffuse $6-311+G(d)$ and non-diffuse $6-311 G(d)$ system to be $0.000254 \%$ (Scheme S1). Based on the decrease in absolute energy differences between basis sets containing diffuse and non-diffuse functions with increasing size of anionic systems, ${ }^{8}$ we expect this energy difference to be the upper bound of error for the systems discussed in this manuscript.

Scheme S1. Benzotriazole model system to evaluate the energy difference between diffuse and non-diffuse functions.

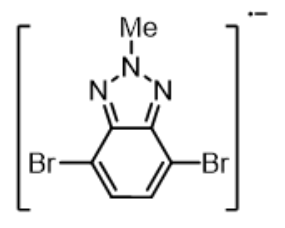

CAM-B3LYP/6-311+G(d)/CPCM(THF): -5582.429756

CAM-B3LYP/6-311G(d)/CPCM(THF): -5582.415587

$\triangle \mathrm{E}: 0.000254 \%$ 


\section{MALDI-TOF-MS of P1}

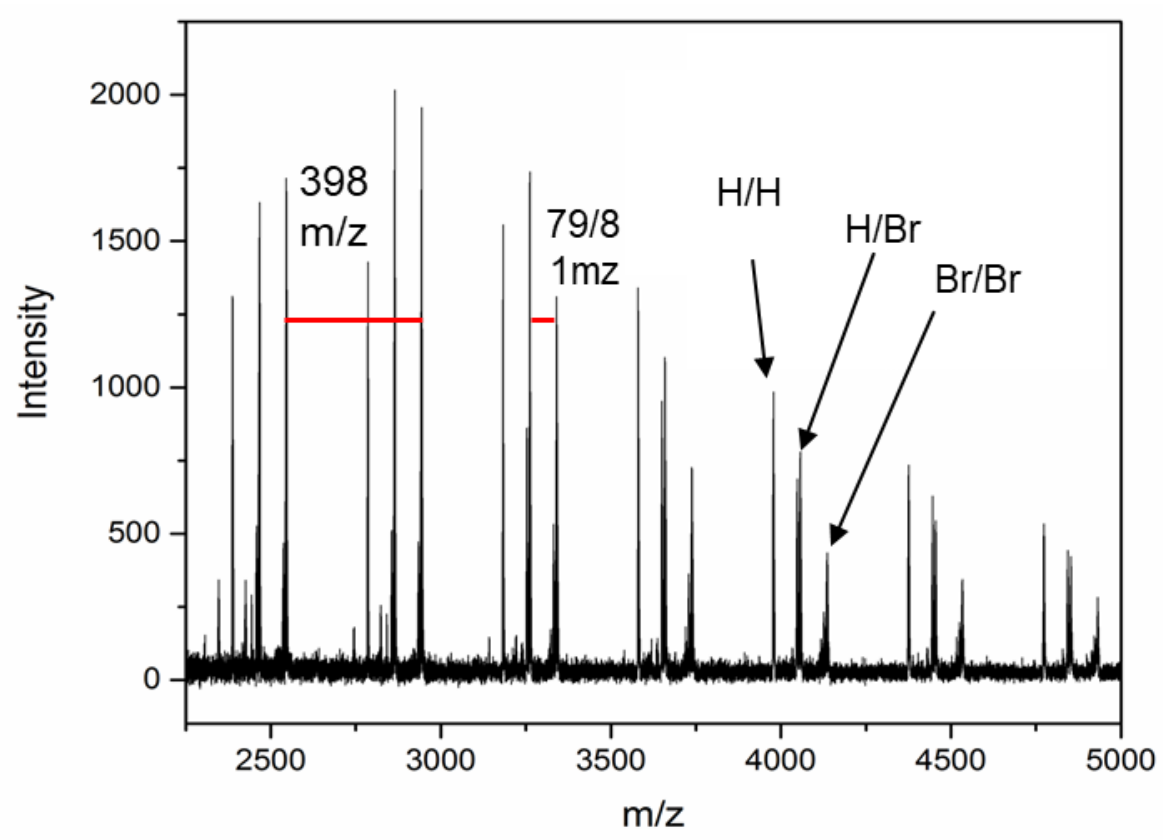

Figure S2: MALDI-TOF of P1 polymerized using conditions prepared from photopolymerization conditions ${ }^{1}$. Spacing between monomer repeat units $(398 \mathrm{~m} / \mathrm{z})$ and mixture of chain-ends are annotated. 


\section{End-capping experiment details}

To determine the chain end of growing polymers in this mechanism, an end-capping experiment was designed with 2,6-difluorobenzaldehyde. The end cap was chosen as an end cap to meet two criteria: First, it should be an electrophile suitable for nucleophilic attack from a Grignard, and second, it should be fluorinated for rapid and unambiguous determination through ${ }^{19} \mathrm{~F}$ NMR.

Sample preparation: In the glovebox, a vial with stir bar was charged with 4,7-dibromo-2-(2octyldodecyl)-2H-benzo[d][1,2,3]triazole (45 mg, $1 \mathrm{Eq}, 81 \mu \mathrm{mol})$, lithium chloride (51 mg, $15 \mathrm{Eq}$, $1.2 \mathrm{mmol})$, and THF $(1.20 \mathrm{~mL})$. A separate vial was charged with $\mathrm{MgBr}_{2} \cdot \mathrm{Et}_{2} \mathrm{O}(21 \mathrm{mg}, 1.00 \mathrm{Eq}$, $81 \mu \mathrm{mol})$ and THF $(400 \mu \mathrm{L})$. The vials were sealed with septum caps and covered with electrical tape. On a Schlenk line, the monomer vial was cooled to $-78{ }^{\circ} \mathrm{C}$ in the dark, then n-butyllithium $(4.7 \mathrm{mg}, 29 \mu \mathrm{L}, 2.5 \mathrm{molar}, 0.90 \mathrm{Eq}, 73 \mu \mathrm{mol})$ was added via air-tight syringe. The lithiate was stirred for $10 \mathrm{~min}$ at $-78{ }^{\circ} \mathrm{C}$, then the etherate solution was injected. The Grignard was stirred in the dark at $-78{ }^{\circ} \mathrm{C}$ for $20 \mathrm{~min}$ and at $23{ }^{\circ} \mathrm{C}$ for $30 \mathrm{~min}$ before irradiation with white LEDs for 4 hours.

While the Grignard solution was irradiated, a flame dried vial with dry THF $(1.20 \mathrm{~mL})$ and freshly distilled 2,6-difluorobenzaldehyde $(11 \mathrm{mg}, 8.7 \mu \mathrm{L}, 1.00 \mathrm{Eq}, 81 \mu \mathrm{mol})$ was degassed through three freeze-pump-thaw cycles.

After 4 hours of polymerization, the aldehyde solution was injected into the sample and the reaction continued to stir under white LEDs at $23^{\circ} \mathrm{C}$ overnight.

The next morning, the polymerization was precipitated into $\mathrm{MeOH}(10 \mathrm{~mL})$ and centrifuged at $5,000 \mathrm{rpm}$ for $10 \mathrm{~min}$. The supernatant was removed to separate the small molecules and concentrated down to a yellow oil. The polymer was taken up in THF and precipitated again into acetone $(10 \mathrm{~mL})$ and centrifuged. The supernatant was again removed and concentrated to isolate the oligomers. The final polymer was an orange solid (5.8 $\mathrm{mg}, 18 \%)$.

Initial NMR screen: First, the isolated small molecules (methanol fraction), oligomers (acetone fraction), and polymer were analyzed with ${ }^{19} \mathrm{~F}$ NMR to determine where the end cap was incorporated. (Figure S5). The methanol fraction contained unreacted end cap, the end capped product, and impurities. No ${ }^{19} \mathrm{~F}$ signal was detected in the oligomers or polymer, suggesting the reactive Grignard in this polymerization is on the monomer.

Purification: The small molecule fraction was purified by preparative thin-layer chromatography (50\% DCM in hex, run twice). The product, (7-bromo-2-(2-octyldodecyl)-2H-benzo[d][1,2,3]triazol4-yl)(2,6-difluorophenyl)methanol, is the seventh band (rf $\sim 0.1$ ), and was scraped off the plate, filtered and rinsed with acetone, then concentrated down to a yellow oil. 
(7-bromo-2-(2-octyldodecyl)-2H-benzo[d][1,2,3]triazol-4-yl)(2,6-difluorophenyl)methanol (3)

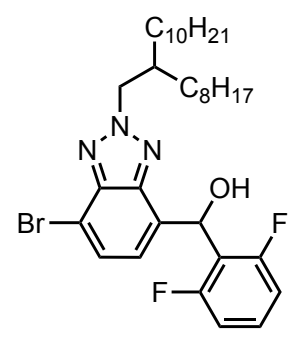

${ }^{1} \mathrm{H}$ NMR $\left(500 \mathrm{MHz}, \mathrm{CDCl}_{3}\right) \delta 7.54(\mathrm{~d}, \mathrm{~J}=7.6 \mathrm{~Hz}, 1 \mathrm{H}), 7.26(\mathrm{~m}, 2 \mathrm{H}), 6.88(\mathrm{t}, \mathrm{J}=8.3 \mathrm{~Hz}, 2 \mathrm{H}), 6.64$ $(\mathrm{d}, \mathrm{J}=7.3 \mathrm{~Hz}, 1 \mathrm{H}), 4.59(\mathrm{~d}, \mathrm{~J}=6.9 \mathrm{~Hz}, 2 \mathrm{H}), 3.53(\mathrm{~d}, \mathrm{~J}=7.4 \mathrm{~Hz}, 1 \mathrm{H}), 2.18(\mathrm{p}, \mathrm{J}=6.2 \mathrm{~Hz}, 1 \mathrm{H})$, 1.25 (dq, J = 17.8, $6.2 \mathrm{~Hz}, 41 \mathrm{H}), 0.86(\mathrm{td}, \mathrm{J}=6.9,2.9 \mathrm{~Hz}, 7 \mathrm{H})$.

${ }^{13} \mathrm{C}$ NMR (126 MHz, $\mathrm{CD}_{2} \mathrm{Cl}_{2}$ ) $\delta$ 161.55, 159.53 (d, J = 7.9 Hz), 143.22, 141.38, 131.02, 129.35 (t, $\mathrm{J}=10.7 \mathrm{~Hz}), 128.00,122.29,111.70-110.66(\mathrm{~m}), 108.74,64.17,60.02,38.46,31.31(\mathrm{~d}, \mathrm{~J}=4.5$ $\mathrm{Hz}), 30.65(\mathrm{~d}, \mathrm{~J}=4.1 \mathrm{~Hz}), 29.70-28.29(\mathrm{~m}), 25.57(\mathrm{~d}, \mathrm{~J}=3.8 \mathrm{~Hz}), 22.09$ (d, J = 3.1 Hz), 13.29 .

${ }^{19} \mathrm{~F} \mathrm{NMR}(470 \mathrm{MHz}, \mathrm{CDCl} 3) \delta-113.51$.

HRMS: $\mathrm{m} / \mathrm{z}$ expected for $\mathrm{C}_{33} \mathrm{H}_{49} \mathrm{BrF}_{2} \mathrm{~N}_{3} \mathrm{O}[\mathrm{M}+\mathrm{H}]^{+}$620.30, measured 620.30 . 


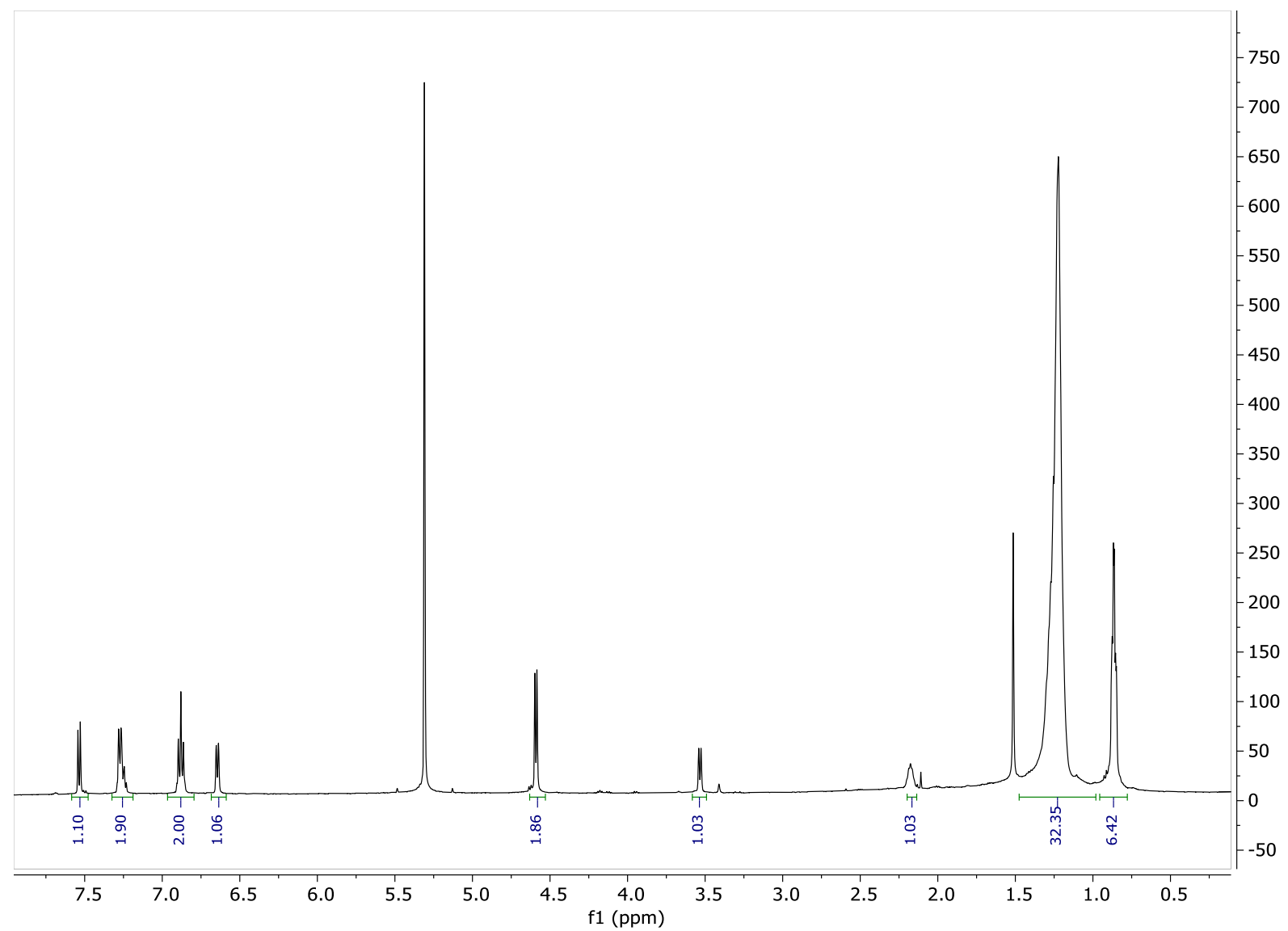

Figure S3: ${ }^{1} \mathrm{H}$ NMR of 3 in $\mathrm{CD}_{2} \mathrm{Cl}_{2}$. 


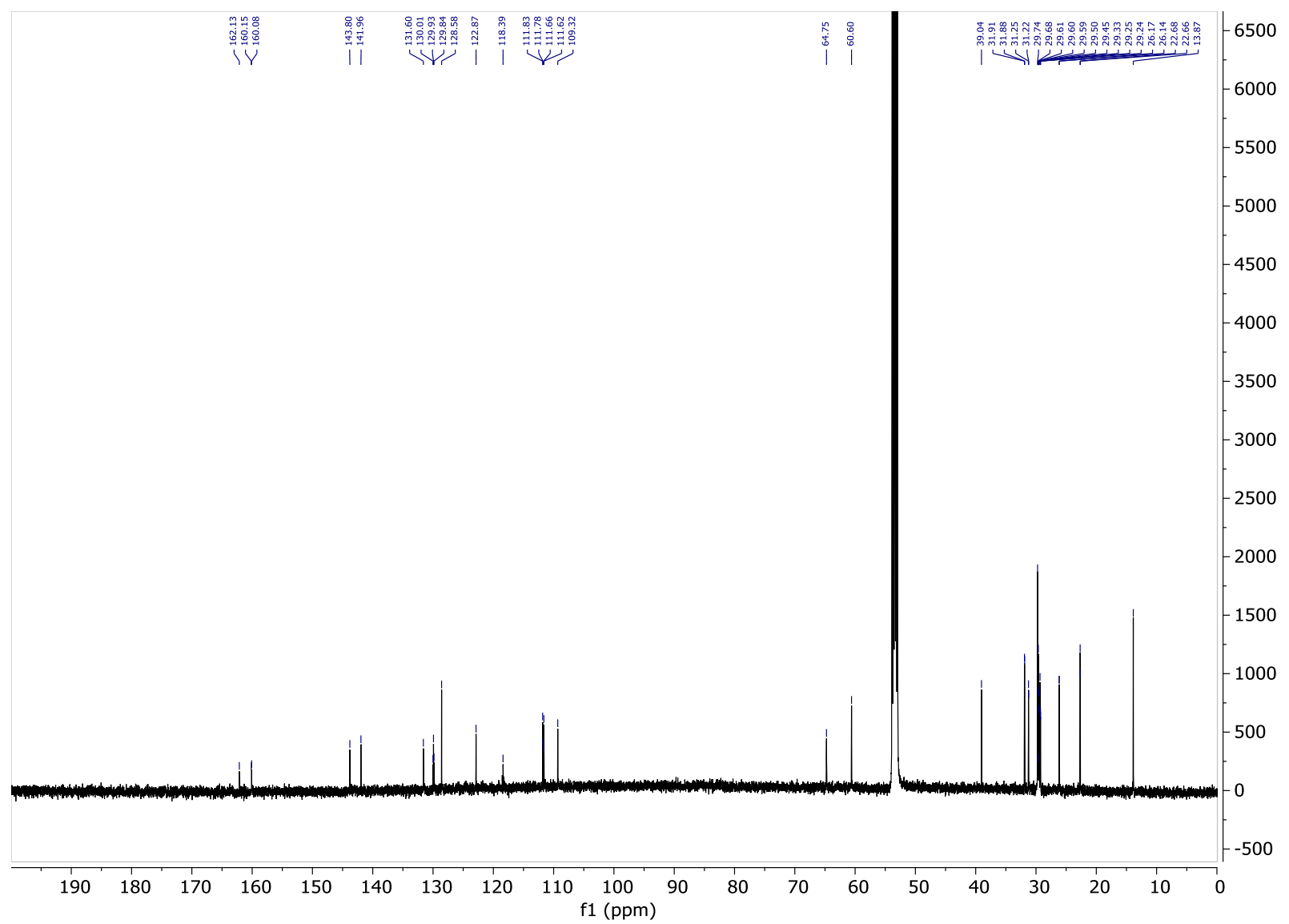

Figure $54:{ }^{13} \mathrm{C}$ NMR of 3 in $\mathrm{CD}_{2} \mathrm{Cl}_{2}$. 


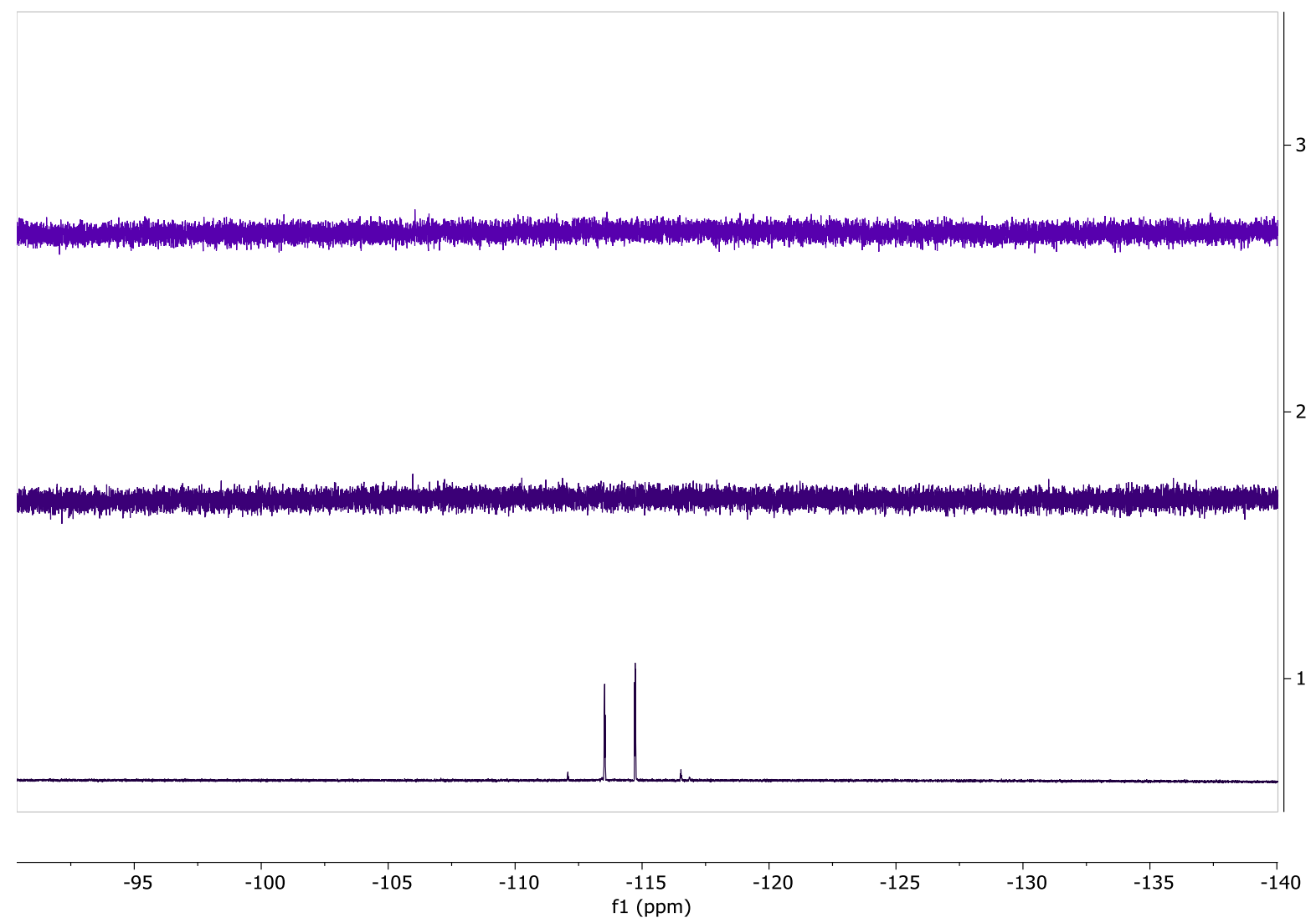

Figure S5: ${ }^{19} \mathrm{~F}$ NMR of the polymer (top), oligomers (middle), and small molecules without further purification (bottom). In the small molecule fraction, the two major peaks are the end-capped product $(-113.51 \mathrm{ppm})$ and unreacted aldehyde $(-114.71 \mathrm{ppm})$. All ${ }^{19} \mathrm{~F}$ spectra were taken with 256 scans. 


\section{Oxidative dimerization experiment details}

To support our hypothesis of monomer oxidative dimerization via trace $\mathrm{O}_{2}$, we subjected a model BTz monomer (SI-1) to the oxidative dimerization conditions developed by Studer and coworkers (Scheme 2). ${ }^{9}$ Consistent with their findings, the aerobic oxidation did not provide sufficient product for isolation and characterization, so TEMPO was used as the oxidant.

Scheme S2. Oxidative dimerization of Grignard reagents.

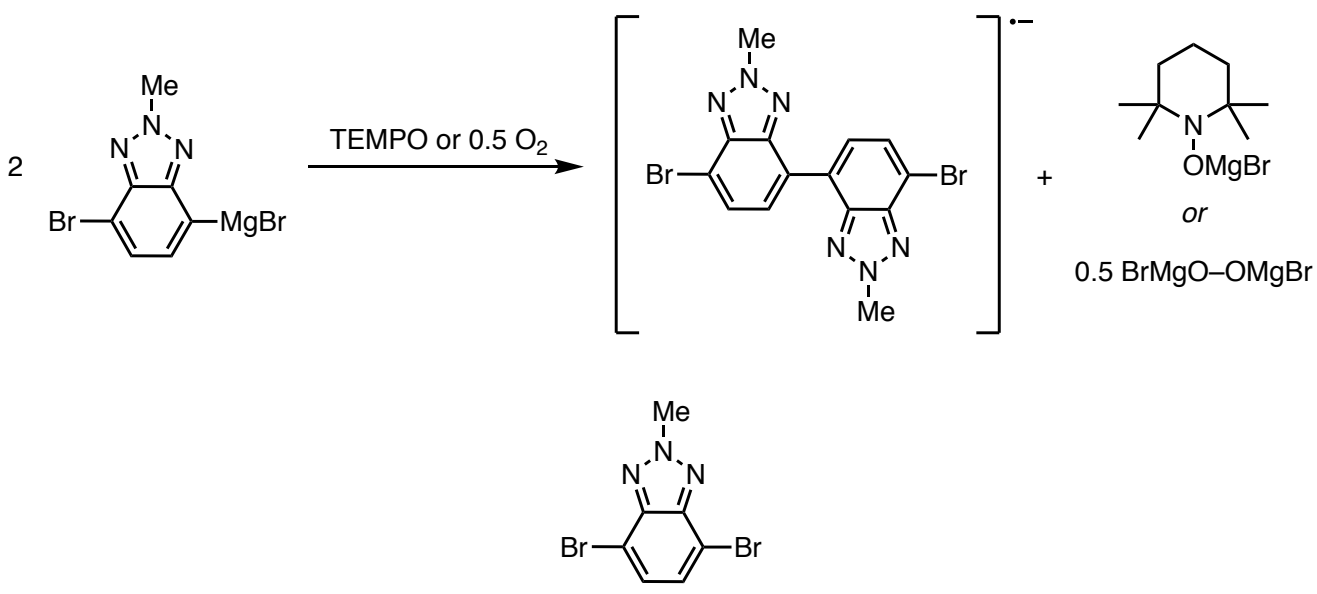

4,7-dibromo-2-methyl-2H-benzo[d][1,2,3]triazole (SI-1): A flame-dried $50 \mathrm{~mL}$ Schlenk flask with stir bar was charged with 4,7-dibromo-2H-benzo[d][1,2,3]triazole (750 mg, 1 eq, 2.71 $\mathrm{mmol})$, cesium carbonate $(1.32 \mathrm{~g}, 1.5 \mathrm{eq}, 4.06 \mathrm{mmol})$, and anhydrous DMF (3.00 $\mathrm{mL})$. The reaction was heated to $40^{\circ} \mathrm{C}$ under $\mathrm{Ar}$, then iodomethane $(500 \mathrm{mg}, 219 \mu \mathrm{L}, 1.3 \mathrm{Eq}, 3.52 \mathrm{mmol})$ was added dropwise. The reaction was stirred at $40^{\circ} \mathrm{C}$ overnight, then the crude material was extracted in EtOAc (3x), washed with water (2x), and brine (1x), and purified by flash column chromatography on silica gel (40\% DCM in hexanes) to yield 4,7-dibromo-2-methyl-2Hbenzo[d][1,2,3]triazole (600 mg, $2.06 \mathrm{mmol}, 76 \%)$ as white needles.

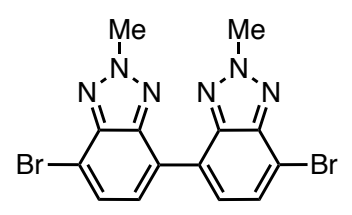

7,7'-dibromo-2,2'-dimethyl-2H,2'H-4,4'-bibenzo[d][1,2,3]triazole (SI-2): A flame-dried 2 dram vial with stir bar was charged with 4,7-dibromo-2-methyl-2H-benzo[d][1,2,3]triazole (50 mg, 1 eq, $0.17 \mathrm{mmol})$ and anhyrous THF $(0.5 \mathrm{~mL})$. The vial was stirred at $23^{\circ} \mathrm{C}$ under Ar before isopropylmagnesium bromide $(25 \mathrm{mg}, 0.31 \mathrm{~mL}, 0.55$ molar, $1.00 \mathrm{eq}, 0.17 \mathrm{mmol}$ ) was added. The Grignard solution was stirred for $10 \mathrm{~min}$ at $23^{\circ} \mathrm{C}$ in the dark, then TEMPO $(29 \mathrm{mg}, 1.08 \mathrm{eq}$, $0.19 \mathrm{mmol}$ ) was added. The reaction was stirred under $\mathrm{Ar}$ for $3 \mathrm{~h}$ at $23^{\circ} \mathrm{C}$, then quenched with saturated $\mathrm{NH} 4 \mathrm{Cl}$. The crude material was extracted in EtOAc (3x), washed with water (2x) and brine $(1 \mathrm{x})$, then dried over magnesium sulfate and concentrated. The product was purified by preparative thin-layer chromatography (40\% DCM in hexanes). 
${ }^{1} \mathrm{H}$ NMR (500 MHz, Acetone) ס 8.62 (d, J = 7.9 Hz, 1H), 7.85 (d, J = 7.8 Hz, 1H), 4.64 (s, 3H). HRMS: $\mathrm{m} / \mathrm{z}$ expected for $[\mathrm{M}+\mathrm{H}]^{+} 420.94$, measured 420.94 .

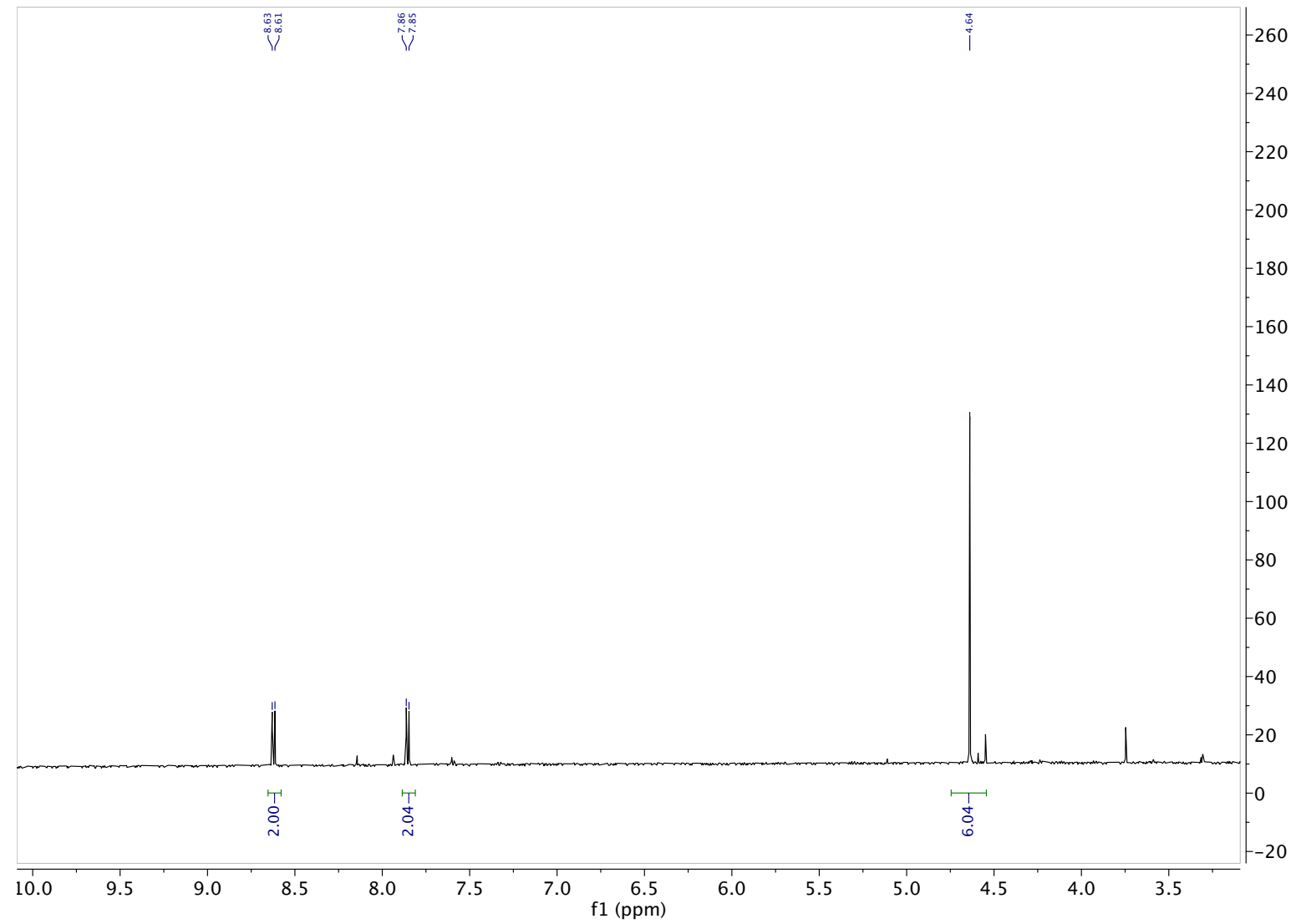

Figure S6: ${ }^{1} \mathrm{H}$ NMR of SI-2 in acetone- $\mathrm{d}_{6}$. 


\section{THF- $d_{8}$ polymerization details}

To verify the competitive $S_{\mathrm{RN}}$ 1-type termination pathway suggested for electron-rich monomers, control polymerizations were conducted in THF-d $\mathrm{d}_{8}$ to search for incorporation of deuterium in the recovered dimers and oligomers.

Thiophene polymerization: In the glovebox, two vials with stir bars were each charged with 2,5dibromo-3-hexylthiophene (1.0 eq, $122 \mu \mathrm{mol}$ ), lithium chloride (15 eq, $1.83 \mathrm{mmol}$ ), and $1.125 \mathrm{~mL}$ of $\mathrm{THF}_{-} \mathrm{d}_{8}$. A separate vial was charged with $\mathrm{MgBr}_{2} \cdot \mathrm{Et}_{2} \mathrm{O}(1.0 \mathrm{eq}, 122 \mu \mathrm{mol})$ and $0.375 \mathrm{~mL}$ of THF$\mathrm{d}_{8}$. Both vials were sealed with septum caps and removed from the glovebox. The thiophene vial was chilled to $-78{ }^{\circ} \mathrm{C}$ in the dark before n-butyllithium (0.9 eq, 2.5 molar, $\left.110 \mu \mathrm{mol}\right)$ was charged. The reaction was stirred at $-78{ }^{\circ} \mathrm{C}$ for $10 \mathrm{~min}$ before the etherate solution was injected into the thiophene vial. The reaction was stirred at $-78{ }^{\circ} \mathrm{C}$ for $20 \mathrm{~min}$, then at $23{ }^{\circ} \mathrm{C}$ for 30 min before irradiation with white LEDs for 24 hours. The reactions were quenched by $\mathrm{MeOH}$. No polymer was obtained by serial precipitation into $\mathrm{MeOH}$, then acetone twice. The $\mathrm{MeOH}$ supernatant was concentrated in vacuo for MALDI-TOF analysis of oligomers and ${ }^{2} \mathrm{H} N M R$ in $\mathrm{MeOH}$.

Benzotriazole polymerization: In the glovebox, two vials with stir bars were each charged with 4,7-dibromo-2-(2-octyldodecyl)-2H-benzo[d][1,2,3]triazole (30 mg, $1 \mathrm{eq}, 54 \mu \mathrm{mol}$ ) and lithium chloride (34 mg, $15 \mathrm{eq}, 0.81 \mathrm{mmol}$ ). Separate vials were charged with $\mathrm{MgBr}_{2} \cdot \mathrm{Et}_{2} \mathrm{O}$ (14 mg, $1.00 \mathrm{eq}, 54 \mu \mathrm{mol})$. One set of vials was charged with THF $(0.75$ for benzotriazole vials and $0.25 \mathrm{~mL}$ for etherate salts), and the other set was charged with THF- $\mathrm{d}_{8}(0.75$ for benzotriazole vials and $0.25 \mathrm{~mL}$ for etherate salts). The vials were capped and sealed, then removed from the glovebox. The benzotriazole vials were stirred at $-78{ }^{\circ} \mathrm{C}$ in the dark before n-butyllithium (3.1 $\mathrm{mg}, 19 \mu \mathrm{L}, 2.5$ molar, $0.90 \mathrm{eq}, 48 \mu \mathrm{mol}$ ) was charged. The reactions were stirred at $-78{ }^{\circ} \mathrm{C}$ for $10 \mathrm{~min}$ before the etherate solutions were injected in their respective reactions. The reactions were stirred in the dark at $-78{ }^{\circ} \mathrm{C}$ for $20 \mathrm{~min}$, then at $23{ }^{\circ} \mathrm{C}$ for 30 min before irradiation with white LEDs for 24 hours. The polymerizations were quenched by $\mathrm{MeOH}$. The polymers were isolated by serial precipitation into $\mathrm{MeOH}$, then acetone twice. The $\mathrm{MeOH}$ supernatant was concentrated in vacuo for MALDI-TOF analysis of oligomers and ${ }^{2} \mathrm{H} N M R$ in $\mathrm{MeOH}$.

THF reaction: Yield 40\%, Mn: 11.6 kg/mol, Đ: 1.3

THF $-\mathrm{d}_{8}$ reaction: Yield: $42 \%, \mathrm{Mn}: 11.0 \mathrm{~kg} / \mathrm{mol}$, Đ: 1.3 

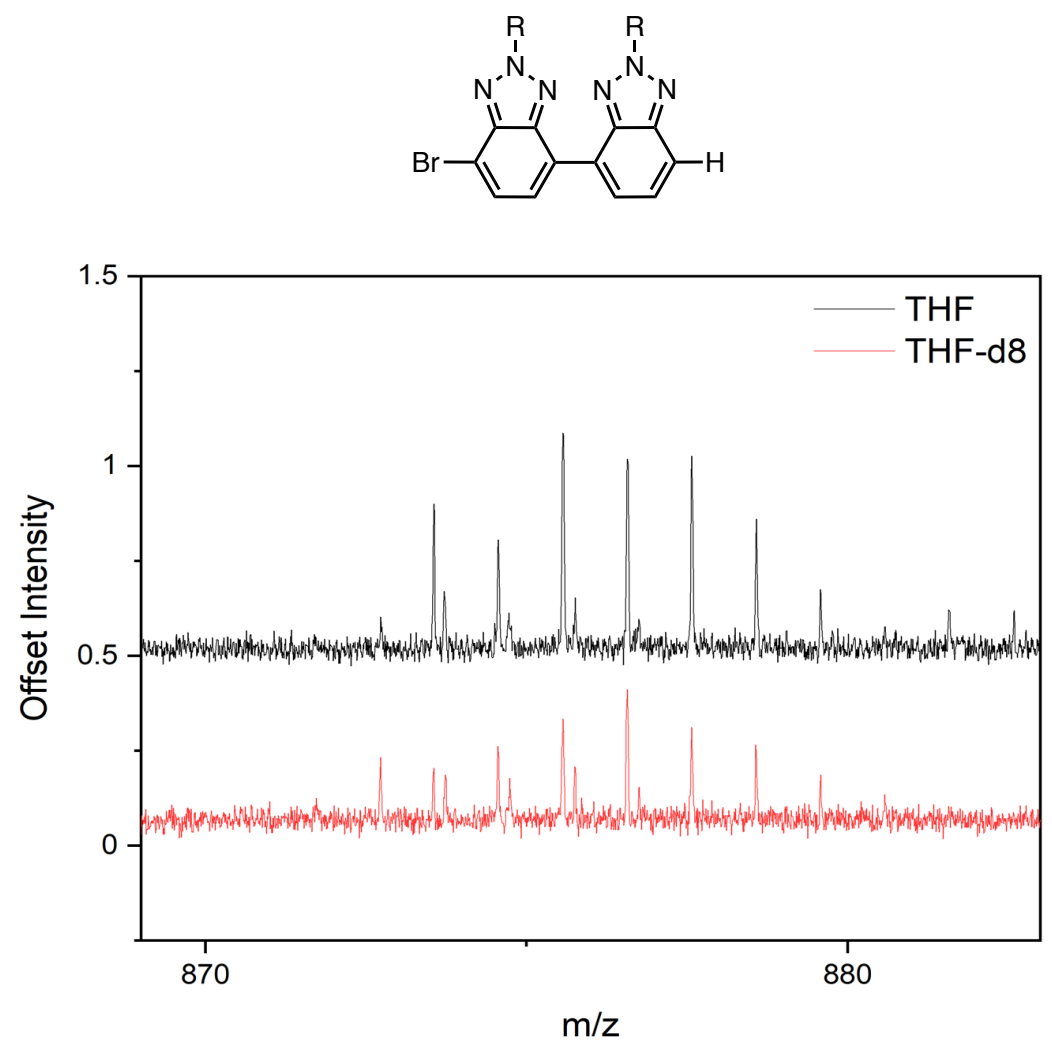

Figure S7: MALDI-TOF of recovered dimers from P1 control polymerizations in THF (black) and

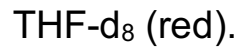




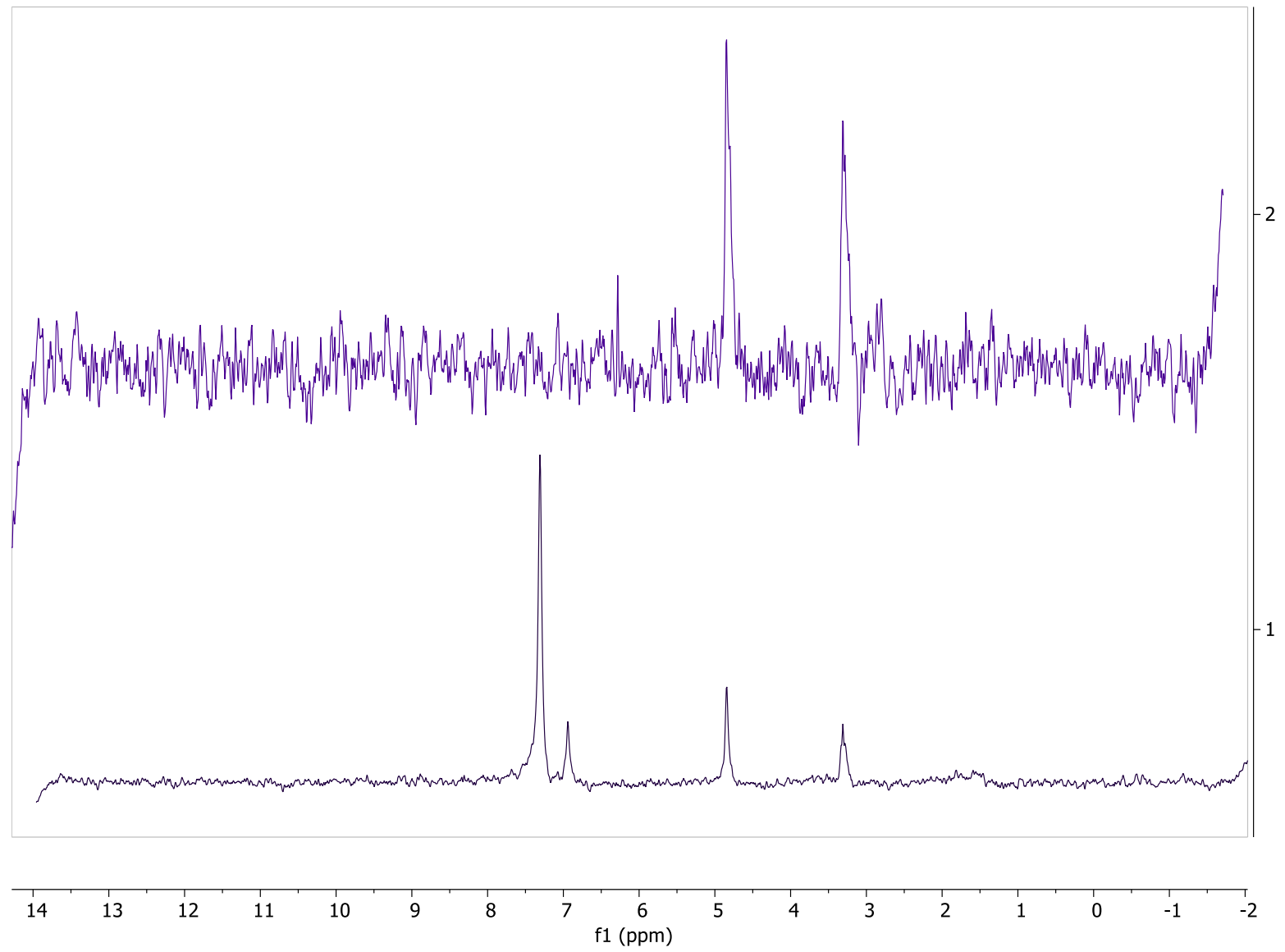

Figure S8: ${ }^{2} \mathrm{H}$ NMR in MeOH of oligomers obtained from photopolymerization of 2-MgBr in THF (top) and THF-d 8 (bottom). The spectra were obtained after allowing the instrument to lock on a control sample in deuterated methanol to improve the signal-to-noise ratio of the ${ }^{2} \mathrm{H}$ signals. 
Spin density maps of calculated structures

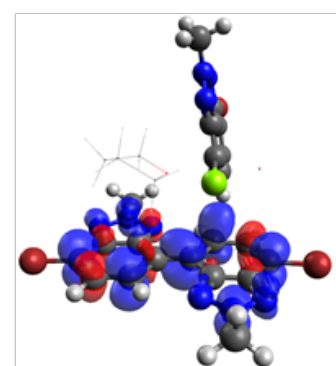

6

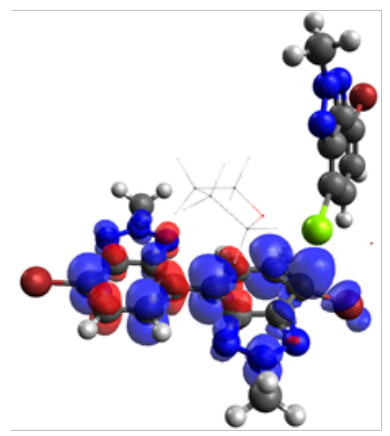

8

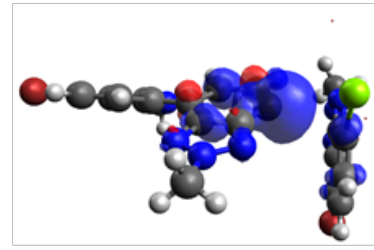

TS-3

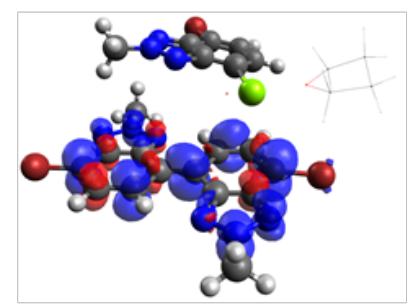

7

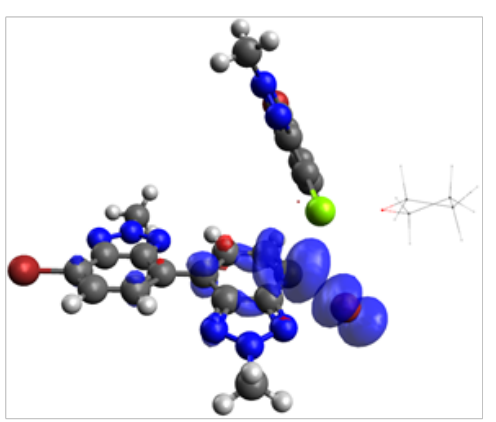

TS-2

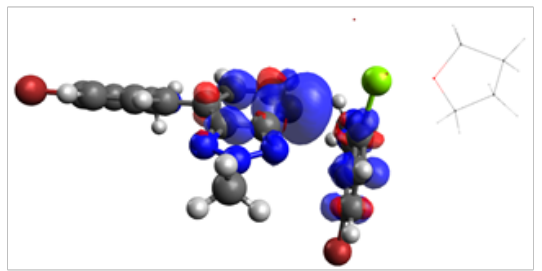

10

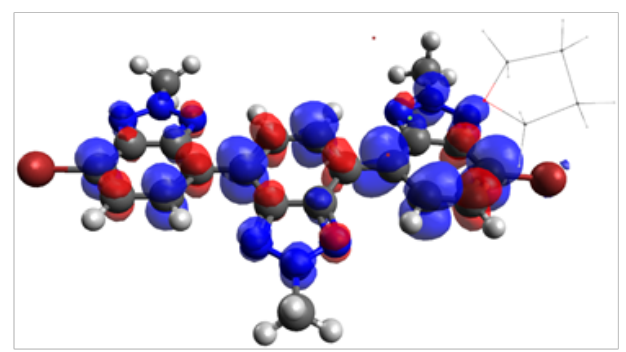

11

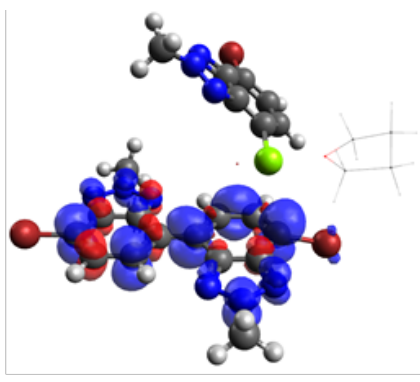

TS-1

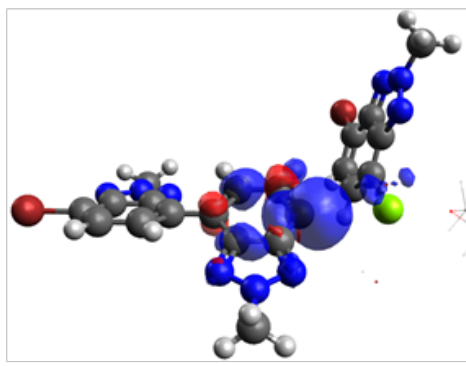

9

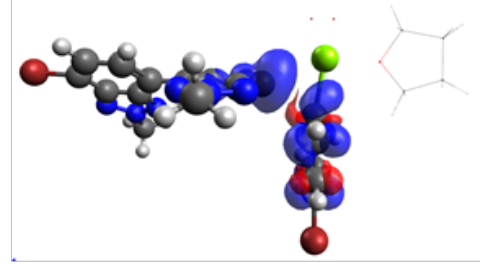

TS-4

Figure S9: Spin density maps of benzotriazole dimer radical anion structures. Isovalue 0.002 used for surface rendering. THF and Mg-coordinated bromines are rendered in skeletal frame for clarity. 
a)

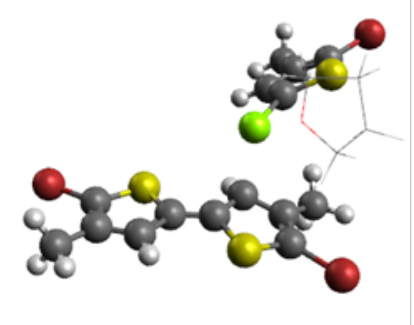

$\mathrm{C}-\mathrm{Br}$

$1.89 \AA$

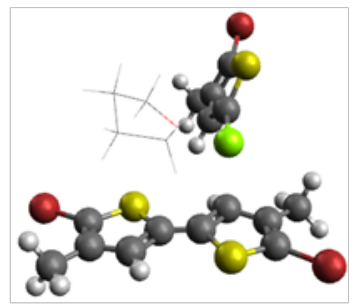

$\mathrm{C}-\mathrm{Br}$

$1.89 \AA$

b)

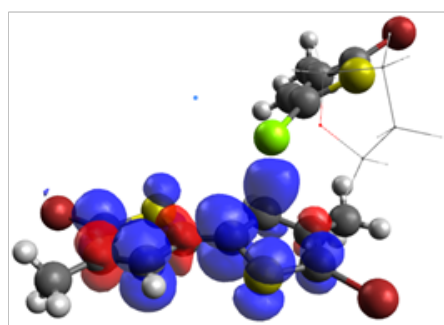

carbon centered coordination conformer 1

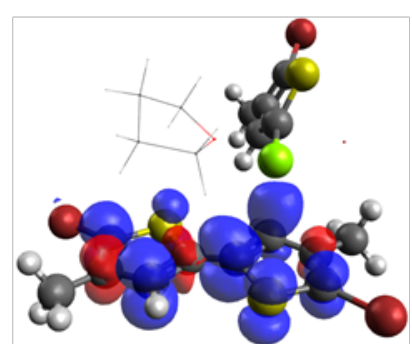

carbon centered coordination conformer 2

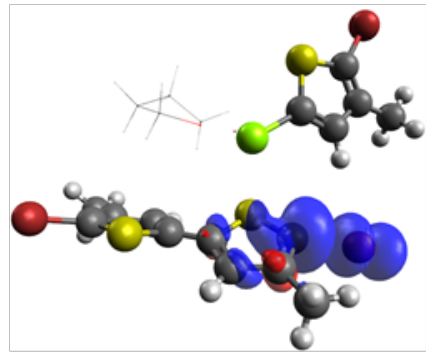

sulfur centered coordination

Figure S10: (a) Structures of thiophene dimer radical anions with carbon-centered Grignard monomer coordination. THF and Mg-coordinated bromine atoms are rendered in wire frame for clarity. (b) Spin density maps of thiophene dimer radical anion structures. Isovalue 0.002 used for surface rendering. THF and Mg-coordinated bromines are rendered in skeletal frame for clarity. 
HOMO and LUMO of P1 oligomers

a)

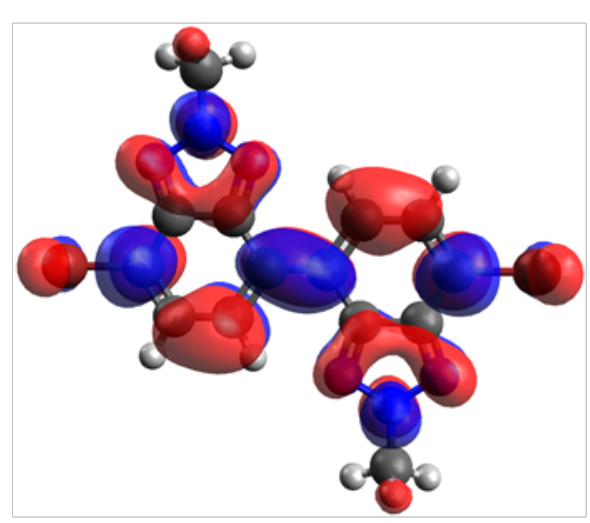

b)

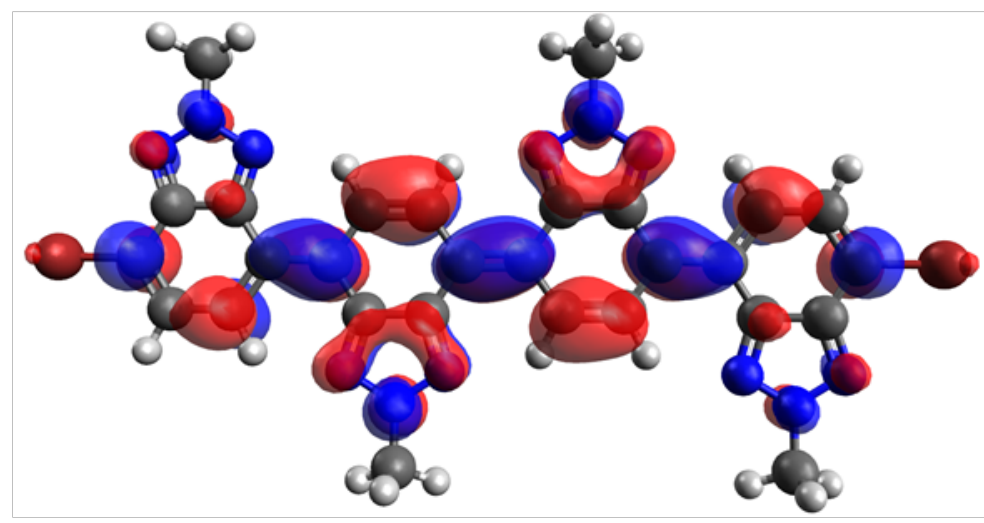

c)

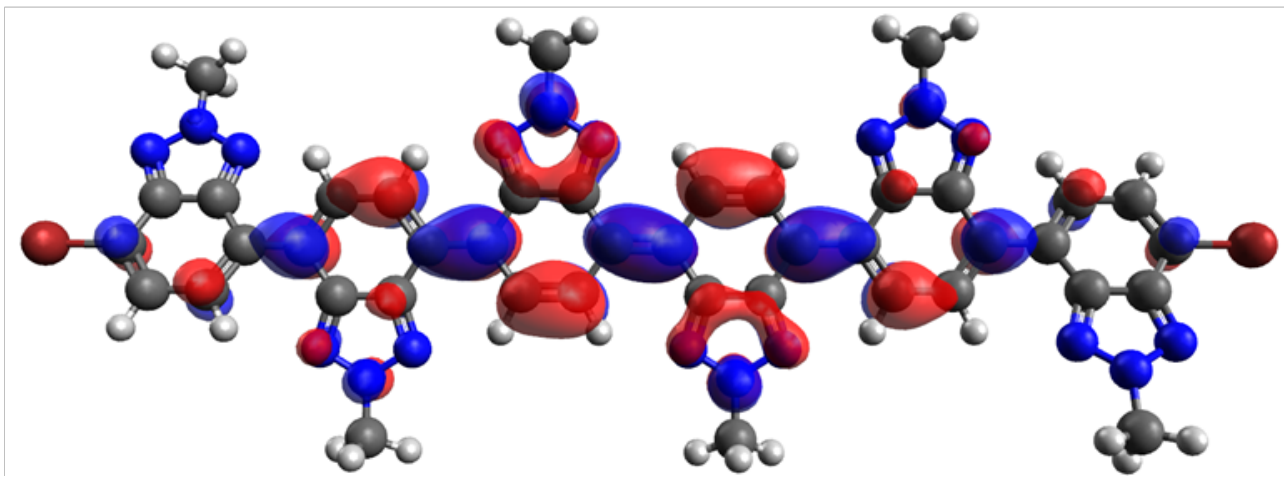


d)

e)

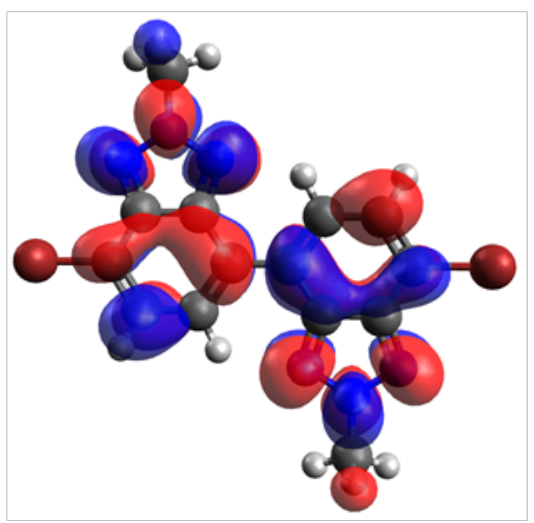

f)
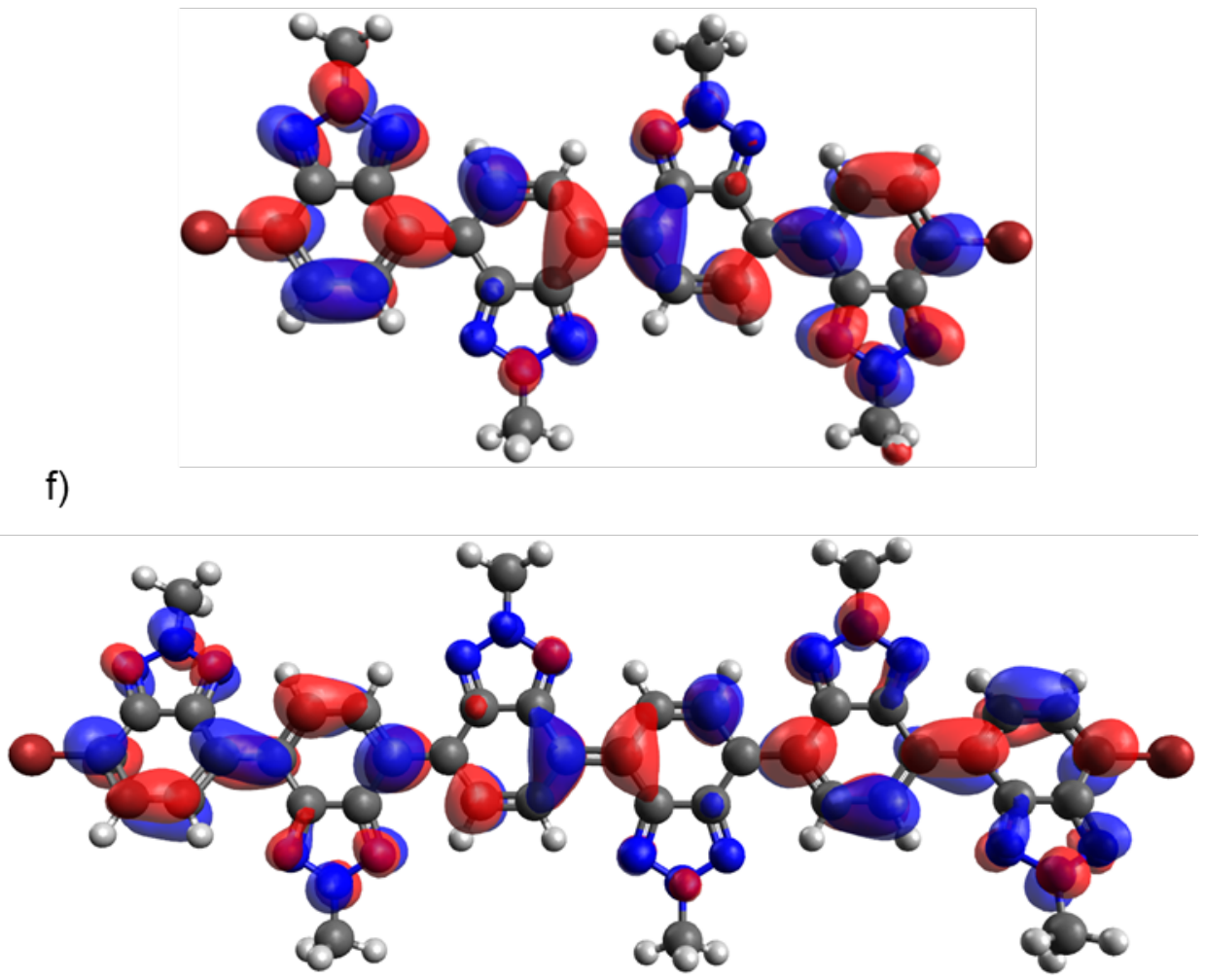

Figure S11: HOMOs of P1 (a) dimer, (b) tetramer, and (c) hexamer radical anions. LUMOs of P1 (d) dimer, (e) tetramer, and (f) hexamer radical anions. 
Additional details for monomer-coordinated hexamer structures

a) Oligomer $\mathrm{D}_{0} \mathrm{HOMO}$

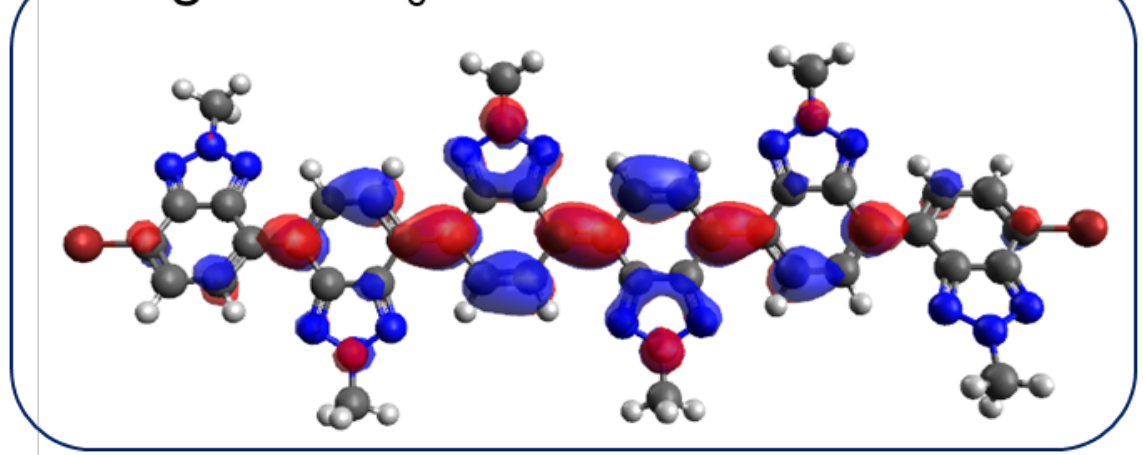

b) Ring 1 coordination

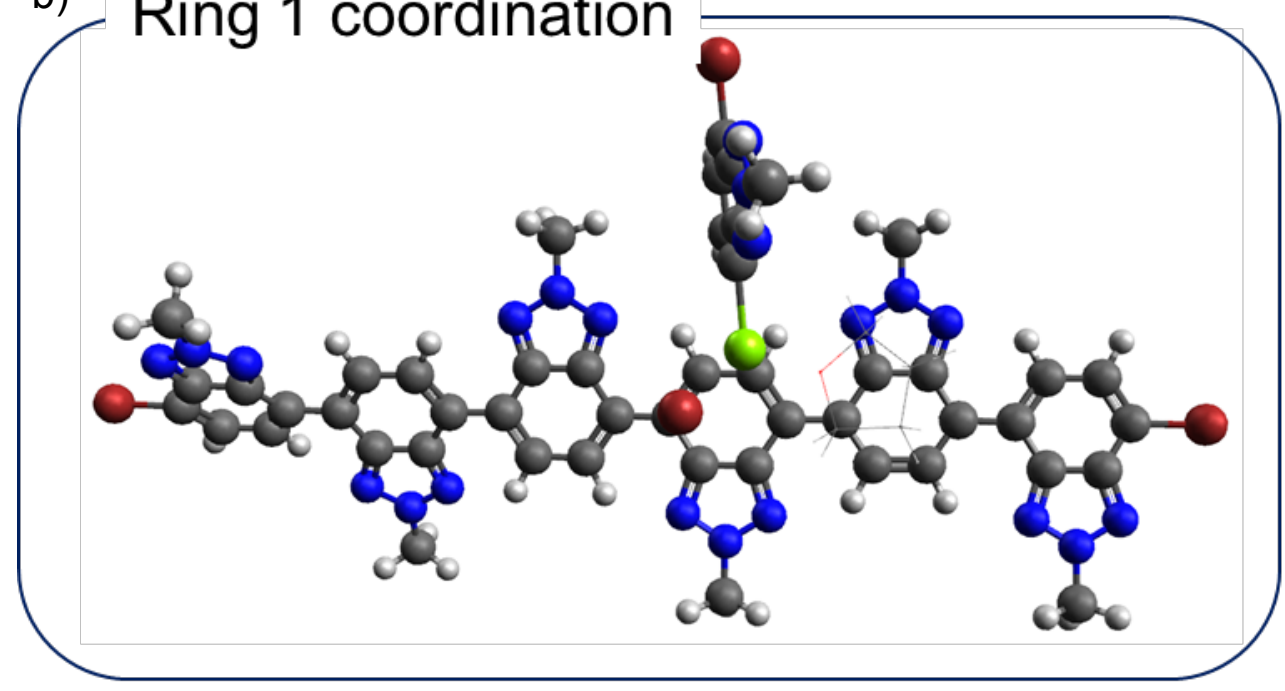

c) Ring 2 coordination

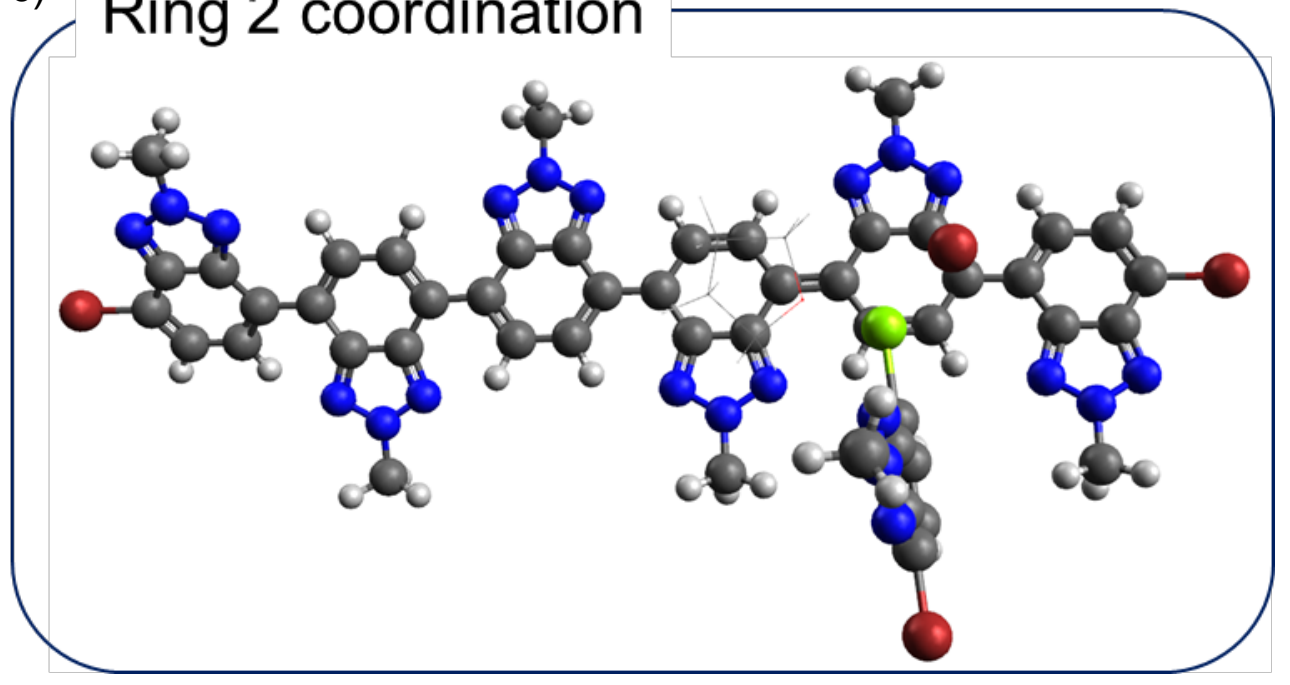




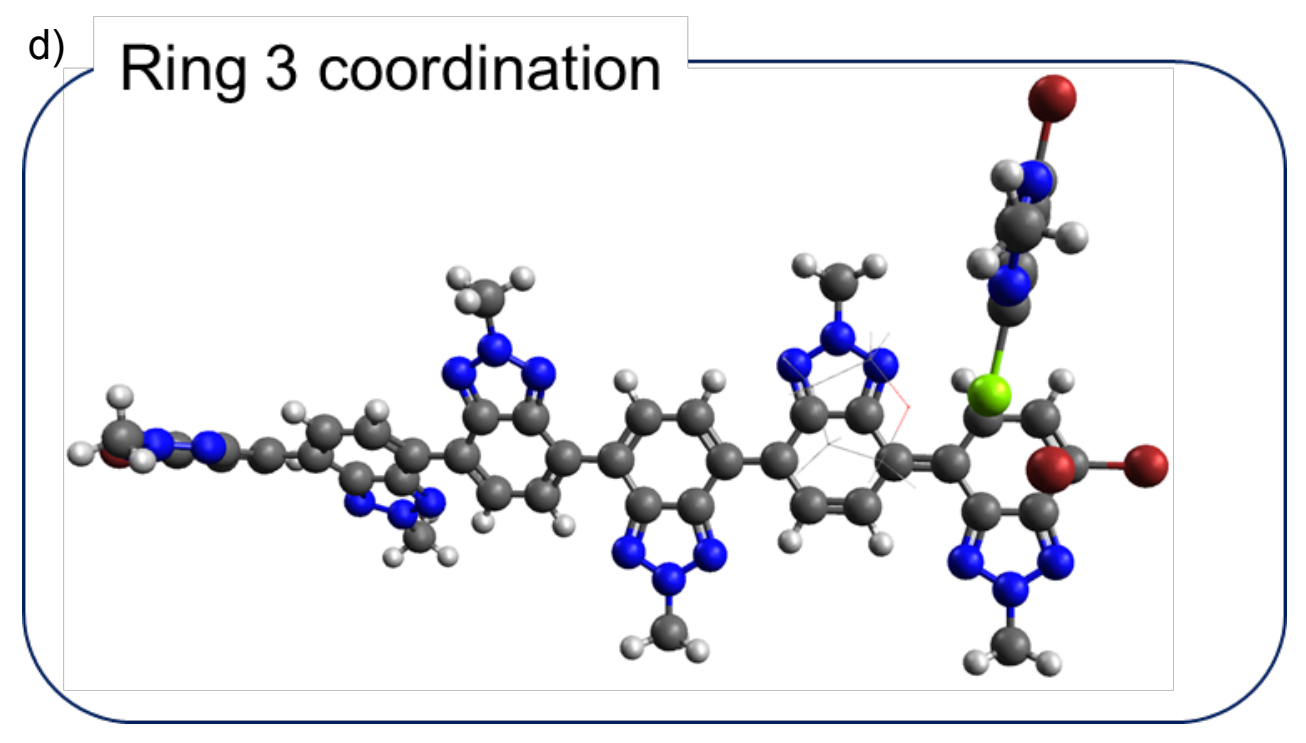

Figure S12: Monomer-coordinated hexamer structures (b-d). Hexamer oligomer $\mathrm{D}_{0} \mathrm{HOMO}$ shown (a) to illustrate the relationship between monomer binding and the HOMO electron density in the ground state. 

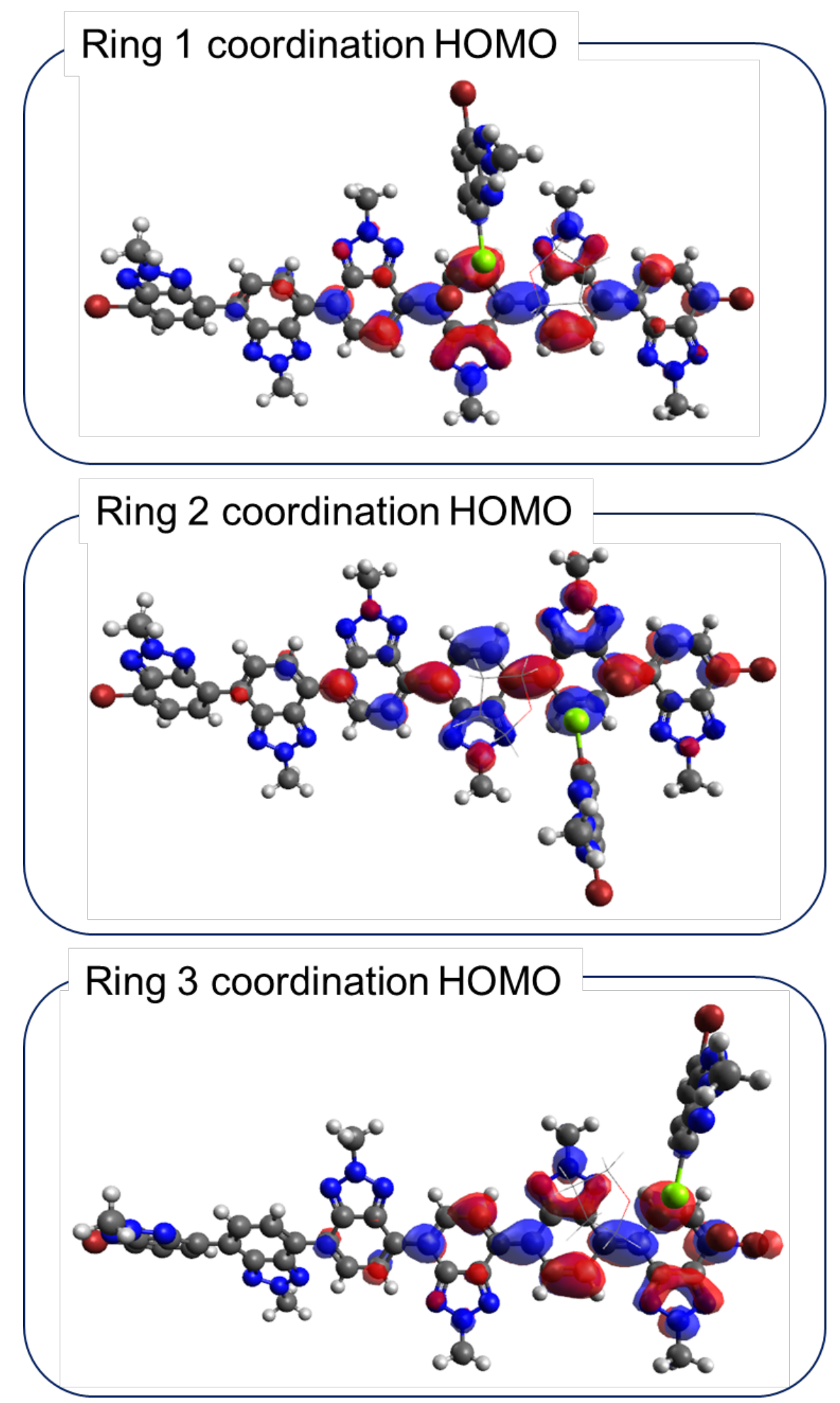

Figure S13: HOMOs of monomer-coordinated hexamer structures. 
Scheme S3: (a) Hexamer coordination naming convention. (b) Carbon bond labeling scheme used for analysis in Table S1.

a)

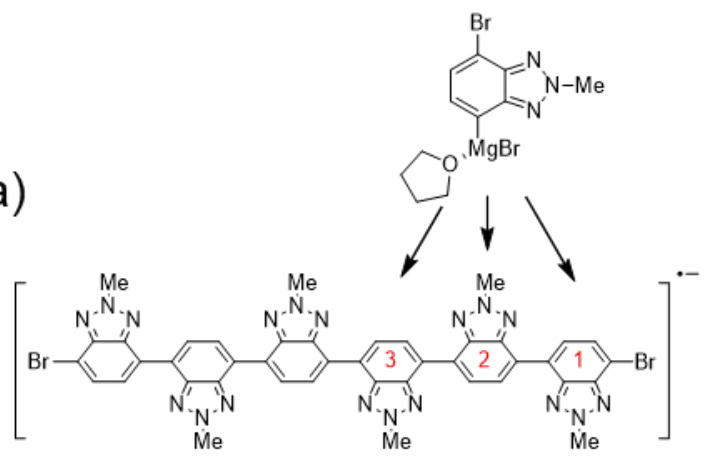

b)

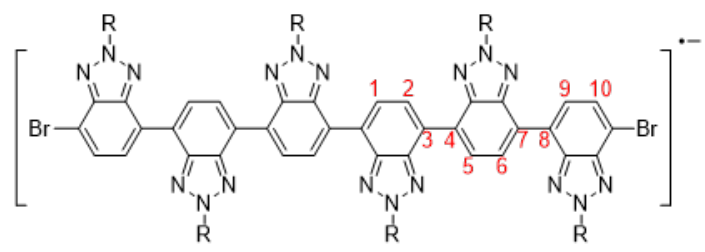

Table S1: Bond lengths in the hexamer chain with monomer coordination at different sites (from computed structures in Scheme S3).

\begin{tabular}{|l|l|l|l|l|}
\cline { 2 - 5 } \multicolumn{1}{c|}{} & Oligomer & $\begin{array}{l}\text { Ring 3 } \\
\text { coordination }\end{array}$ & $\begin{array}{l}\text { Ring 2 } \\
\text { coordination }\end{array}$ & $\begin{array}{l}\text { Ring 1 } \\
\text { coordination }\end{array}$ \\
\hline Bond 1-2 & $1.389 \AA$ & $1.399 \AA$ & $1.390 \AA$ & $1.404 \AA$ \\
\hline Bond 2-3 & $1.402 \AA$ & $1.425 \AA$ & $1.410 \AA$ & $1.397 \AA$ \\
\hline Bond 3-4 & $1.449 \AA$ & $1.428 \AA$ & $1.426 \AA$ & $1.445 \AA$ \\
\hline Bond 4-5 & $1.394 \AA$ & $1.408 \AA$ & $1.425 \AA$ & $1.408 \AA$ \\
\hline Bond 5-6 & $1.405 \AA$ & $1.393 \AA$ & $1.402 \AA$ & $1.384 \AA$ \\
\hline Bond 6-7 & $1.383 \AA$ & $1.397 \AA$ & $1.394 \AA$ & $1.414 \AA$ \\
\hline Bond 7-8 & $1.467 \AA$ & $1.458 \AA$ & $1.455 \AA$ & $1.426 \AA$ \\
\hline Bond 8-9 & $1.381 \AA$ & $1.388 \AA$ & $1.399 \AA$ & $1.426 \AA$ \\
\hline Bond 9-10 & $1.481 \AA$ & $1.415 \AA$ & $1.415 \AA$ & $1.419 \AA$ \\
\hline
\end{tabular}

Table S2: Calculated thermodynamics for monomers bound to different carbon sites on the hexamer radical anion.

\begin{tabular}{|l|c|c|c|c|c|c|}
\cline { 2 - 7 } \multicolumn{1}{c|}{} & $\mathrm{H}(\mathrm{kcal} / \mathrm{mol})$ & $\Delta \mathrm{H}(\mathrm{kcal} / \mathrm{mol})$ & $\mathrm{S}(\mathrm{kcal} / \mathrm{mol})$ & $\begin{array}{c}\Delta \mathrm{S} \\
(\mathrm{kcal} / \mathrm{mol})\end{array}$ & $\mathrm{G}(\mathrm{kcal} / \mathrm{mol})$ & $\begin{array}{c}\Delta \mathrm{G} \\
(\mathrm{kcal} / \mathrm{mol})\end{array}$ \\
\hline $\begin{array}{l}\text { Ring 1 } \\
\text { coordination }\end{array}$ & -8638013.78 & 0.00 & 95.93 & 0.00 & 8638109.71 & 0.00 \\
\hline $\begin{array}{l}\text { Ring 2 } \\
\text { coordination }\end{array}$ & -8638020.96 & -7.19 & 109.10 & 13.16 & $\begin{array}{c}- \\
8638130.06\end{array}$ & -20.35 \\
\hline $\begin{array}{l}\text { Ring 3 } \\
\text { coordination }\end{array}$ & -8638021.21 & -7.43 & 105.86 & 9.92 & 8638127.06 & -17.35 \\
\hline
\end{tabular}




\section{Discussion of energy differences in hexamer structures}

In comparing the HOMOs of the hexamer-coordinated monomer structures (Figure S13), we observed that relative to the uncoordinated hexamer and structures coordinated at rings 2 and 3 , coordination of monomer to ring 1 results in a large polarization of the HOMO to the chain end. Moving the excess negative charge from the area of longest effective conjugation to the chain end is unfavorable and likely the origin of the $\sim 7 \mathrm{kcal} / \mathrm{mol}$ enthalpy penalty for binding monomer at the chain end relative to the inner rings (Table S2).

There is an additional entropic penalty for adopting chain-end monomer coordination, $\sim 9.9$ $\mathrm{kcal} / \mathrm{mol}$ relative coordination at inner rings (Table S2). Inspection of the bond lengths along the polymer backbone in the different monomer-coordinated structures (Table S1) shows that the C$\mathrm{C}$ bond connecting rings 1 and 2 (bond 7-8) undergoes significant contraction when the monomer binds ring 1. At the same time, bonds 6-7 and 8-9 elongate. These changes in bond length suggest that the localization of negative charge to the chain end caused by monomer coordination results in more double bond character about the $7-8 \mathrm{C}-\mathrm{C}$ bond as the chain end adopts a quinoidal structure. This restricted rotation at the chain end does not appear to be entropically compensated by conformational loosening within the inner rings. Thus, coordinating a monomer at ring 1 is accompanied by structural changes that result in an entropic penalty.

It should be noted that when computing larger structures, low-frequency modes can contribute significant noise to the calculation of the system entropy. The quasi-rigid-rotor harmonic approximation developed by Grimme ${ }^{7}$ and used in this work shows excellent mitigation of these errors for systems up to 300-400 atoms with typical error reduced to $\sim 2 \mathrm{kcal} / \mathrm{mol}$. As such, the entropy values calculated for the hexamer structures (123 atoms) are unlikely to be the result of numerical noise. 


\section{Excited-state geometry for monomer-coordinated structures}

a)

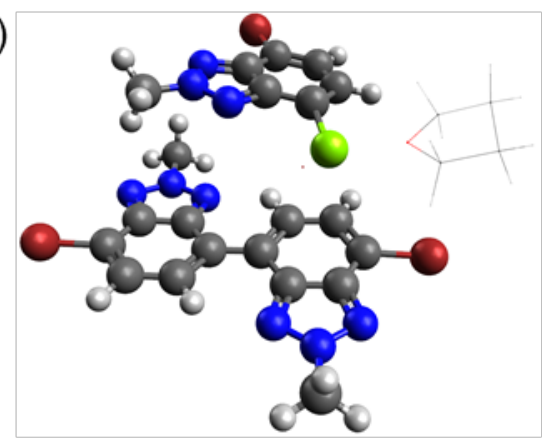

b)

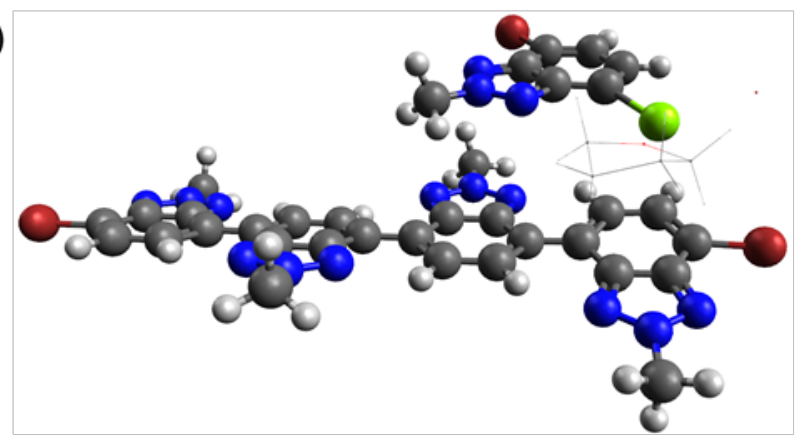

c)

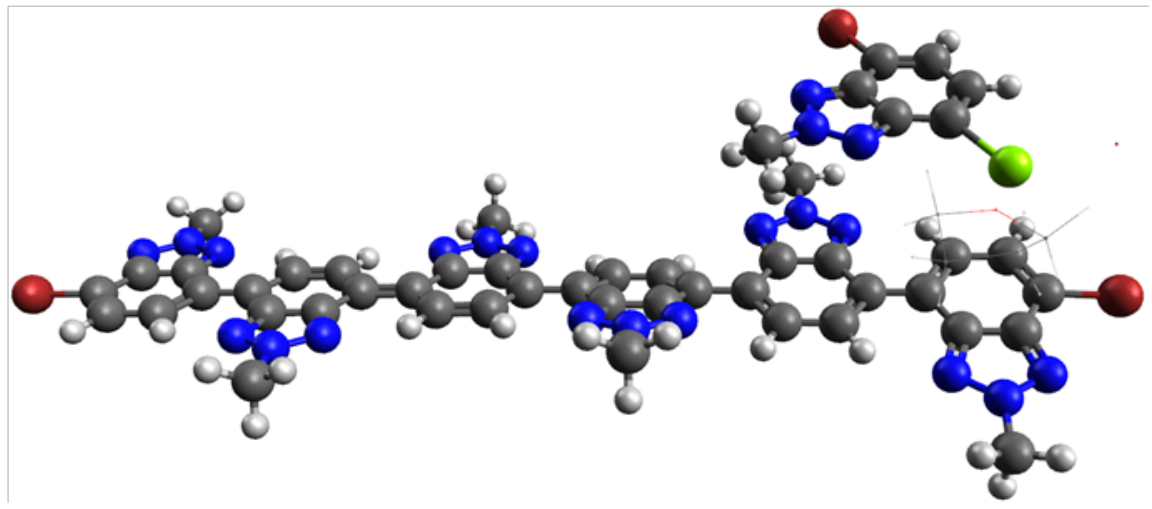

Figure S14: Excited state optimized structures of monomer coordinated to dimer (a) tetramer (b), and hexamer (c) for the P1 system. THF and Mg-coordinated bromines are rendered in skeletal frame for clarity. 


\section{Excited-state lifetime of $\mathbf{P} \mathbf{1}$ radical anion}

\section{Preparation of sodium naphthalenide-doped P1 for transient absorption}

In a nitrogen-filled glovebox, a sample of $3.3 \mathrm{mg}$ of $\mathbf{P 1}\left(\mathrm{M}_{\mathrm{n}}: 10.2 \mathrm{~kg} / \mathrm{mol}, 1.48 \boxminus\right)$ prepared according to literature procedures ${ }^{1}$ was dissolved in $10 \mathrm{~mL}$ of THF. Sodium naphthalenide THF solution $(7.8 \mathrm{mM}, 1 \mathrm{eq})$ was then charged into the $\mathbf{P} 1$ solution and mixed through inversion of the vial. $0.5 \mathrm{~mL}$ of the solution was then transferred into a $1.0 \mathrm{~mm}$ path-length cuvette with septum cap and removed from the glovebox for measurement.

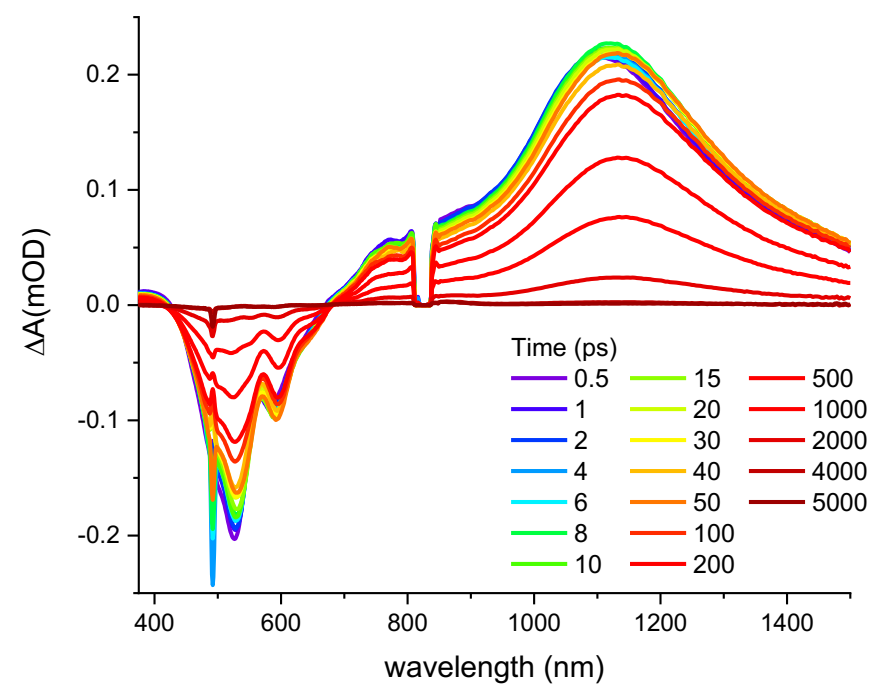

Figure S15: Femtosecond transient absorption (fsTA) data for $\mathbf{P} 1$ radical anion in THF at room temperature, with excitation at $490 \mathrm{~nm}$. The excited state lifetime is $850 \pm 6 \mathrm{ps}$, calculated from wavelength global fitting in Matlab. 


\section{Determination of the quantum yield}

We define quantum yield to be the ratio of the number of monomers consumed to the number of photons absorbed by the reaction solution. Note that the latter quantity includes photons absorbed by radical anion chains, terminated chains, and other nonproductive species. The quantum yield thus includes internal filter effects. This definition of quantum yield has been used in the context of photopolymerizations before. ${ }^{10,11}$

We determined the number of monomers consumed as a function of irradiation time with white LEDs using ${ }^{1} \mathrm{H}$ NMR. These data are shown in Figure S16. In order to extract a rate, we performed a linear fit on the data up to 420 minutes, beyond this point the consumption of monomer no longer followed a linear trend with time.

To determine the number of photons absorbed by the reaction mixture, we measured the emission spectrum and flux of the $4000 \mathrm{~K}$ white LEDs used in the reaction above, and at each time point we measured an absorbance spectrum of the reaction mixture using an Ocean Optics FLAMECHEM UV-Vis. These absorbance spectra are found in Figure S17. We measured the emission spectrum of the LEDs using an Ocean Optics Jaz UV/Vis Spectrophotometer. To convert this unitless emission spectrum into a wavelength-dependent photon flux, we measured the total flux of the white LEDs at the location of the reaction mixture to be $5.3 \pm 0.3 \mathrm{~mW} / \mathrm{cm}^{2}$ using a Thor Labs PM100D Power Meter (Figure S18). We estimated the uncertainty in this flux value by moving the detector up and down in the approximate location of the reaction. This uncertainty is by far the greatest source of uncertainty in our quantum yield determination, so other sources such as NMR or UV-Vis spectroscopy were neglected.

Using the data above, we wrote a MATLAB script to calculate the number of photons absorbed by the reaction solution at each time point and at each measurement wavelength of the LED emission spectrum:

$$
\text { \# photons absorbed }=\left(1-10^{-A}\right)(\Phi \times \text { surface area })
$$

where $A$ is the absorbance of the reaction solution at the relevant wavelength, $\Phi$ is the photon flux in units of photons $/ \mathrm{cm}^{2}$, and "surface area" refers to the surface area of the reaction solution exposed to the LEDs. In the experiment, $3.2 \mathrm{~mL}$ of reaction solution were irradiated in a standard $1 \mathrm{~cm}$ path length cuvette, so the surface area is $3.2 \mathrm{~cm}^{2} \times 4=12.8 \mathrm{~cm}^{2}$. Note that we have assumed that the optical path length in the reaction solution is the same as that in the absorbance measurement, $1 \mathrm{~cm}$. In truth, the average path length would be very difficult to determine. The average path length of a random set of lines through a square of side length $1 \mathrm{~cm}$ is $0.7854 \mathrm{~cm} .{ }^{12}$ However, this value neglects the possible paths that do not lie horizontally, which will increase the length of the average path. Thus, for simplicity we chose to assume the average path length through the cuvette to be $1 \mathrm{~cm}$. Because the absorbance measurement below $560 \mathrm{~nm}$ saturated the detector, we manually removed some false zero values from the raw absorbance data. In these cases, we arbitrarily set the absorbance to 3 . This value corresponds to $99.9 \%$ of photons being absorbed, so this arbitrary value has a negligible affect on the final quantum yield determination. The quantum yield thus calculated starts at $3.9 \%$ at the beginning of the reaction but drops to a plateau of about $1.6 \%$ over the first hour of irradiation (Figure S19). 


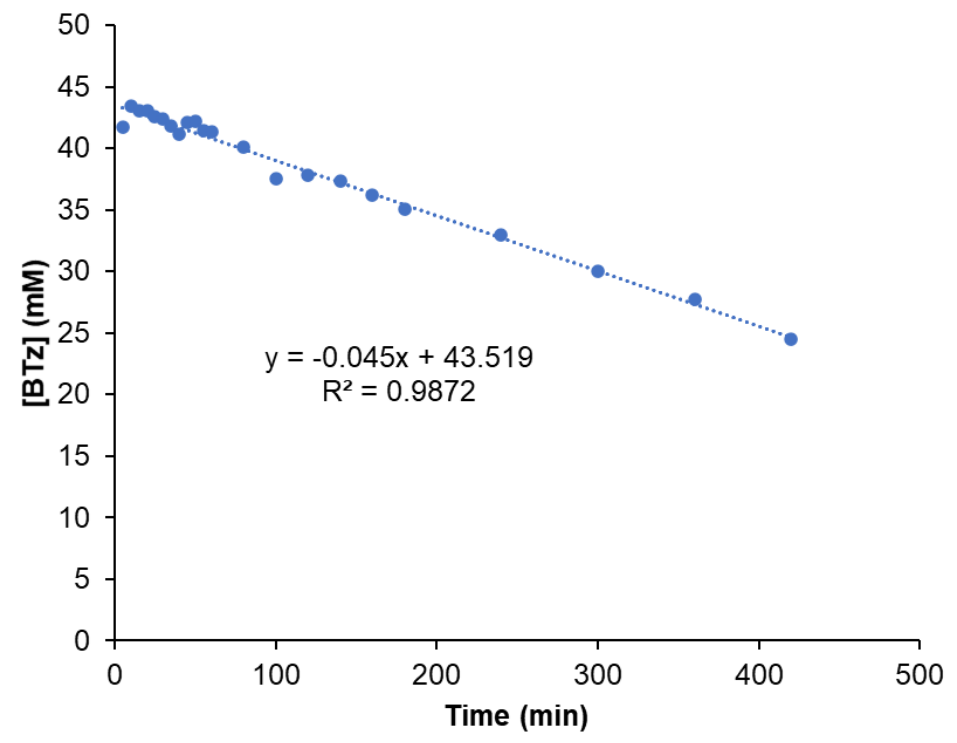

Figure S16. Concentration of 1-MgBr monomer during photopolymerization measured by ${ }^{1} \mathrm{H}$ NMR (relative to trimethoxy benzene internal standard) as a function of irradiation time.

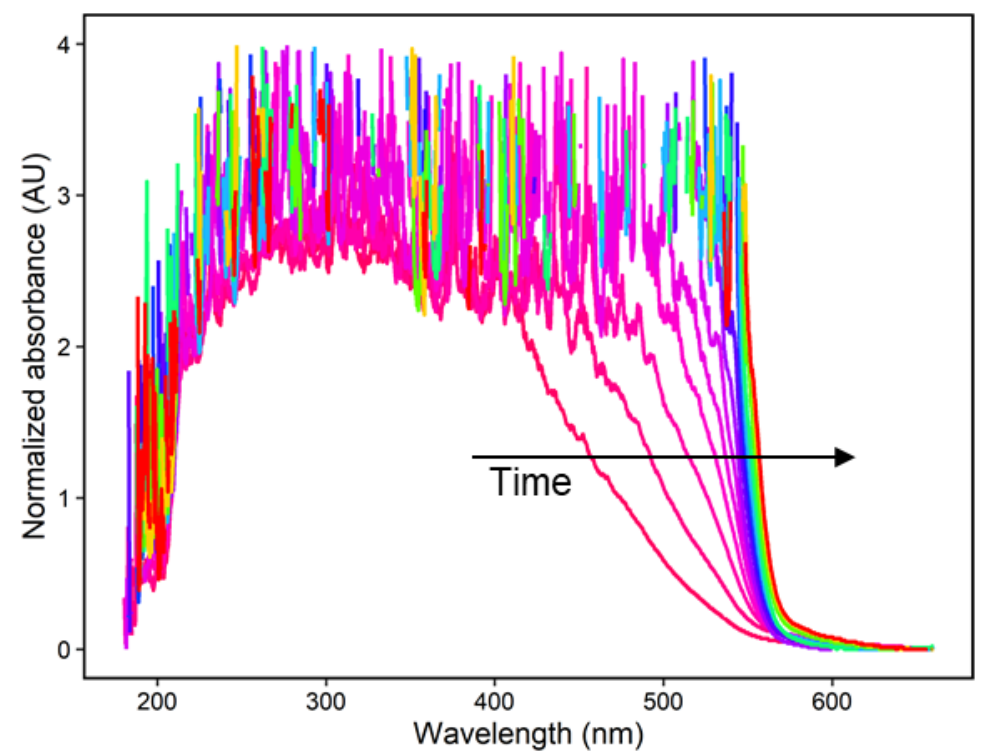

Figure S17. Absorbance spectra of the polymerization of $1-\mathrm{MgBr}$ reaction solution at various irradiation times. 


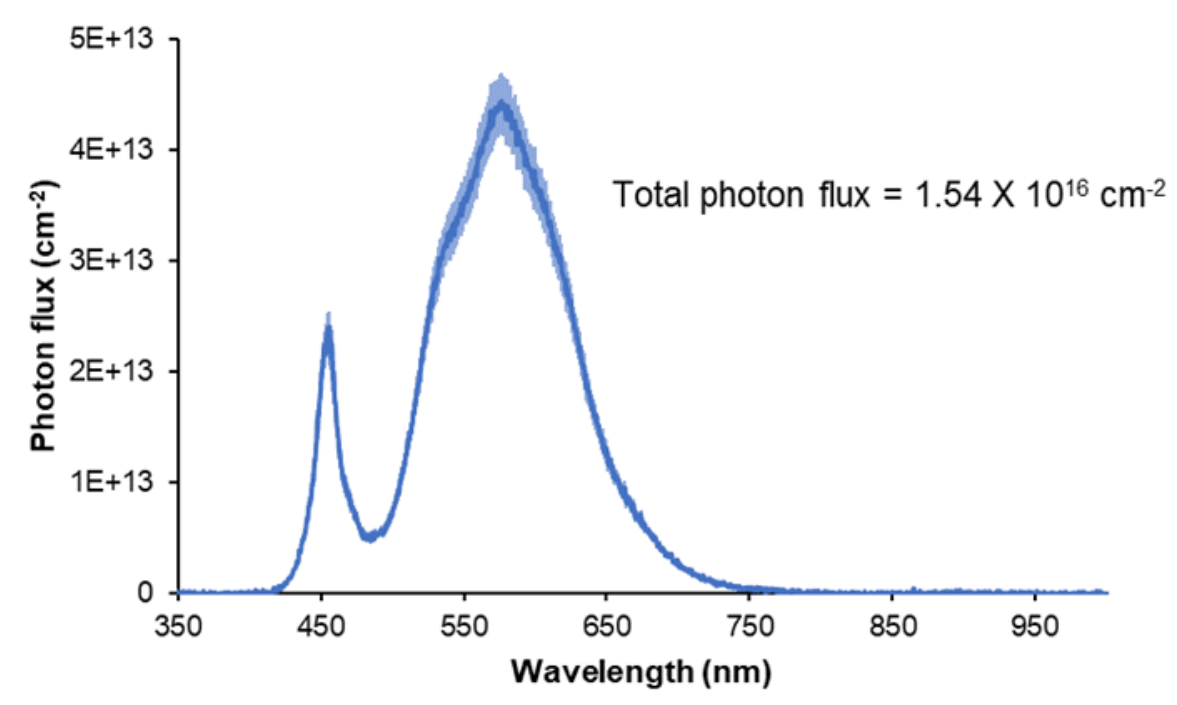

Figure S18. Emission profile of while LEDs converted into photon flux.

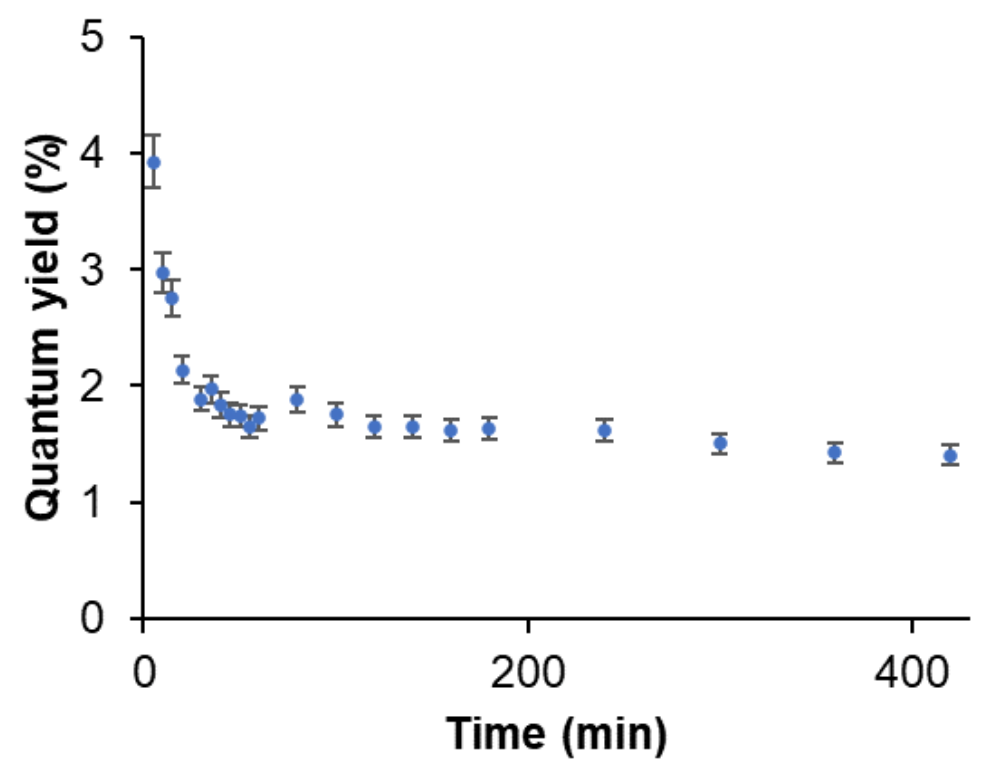

Figure S19. Quantum yield of 1-MgBr polymerization as a function of irradiation time. 


\section{Calculations for aggregation of benzotriazole Grignard monomer}

a)

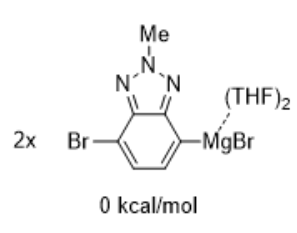

b)

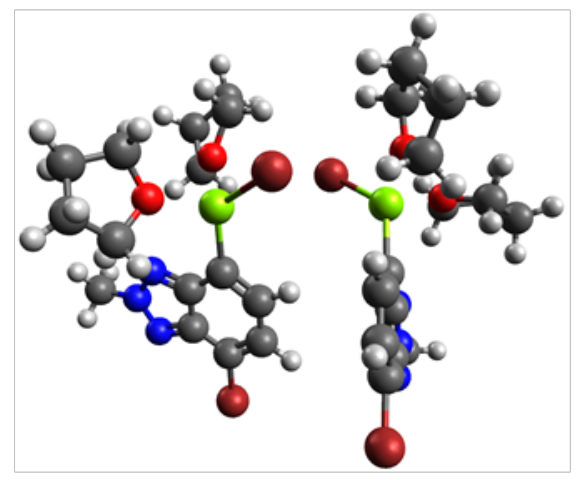

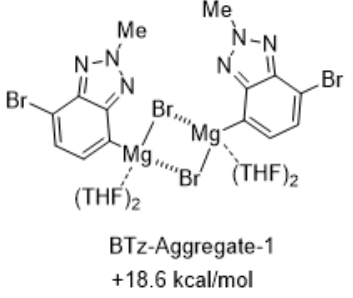

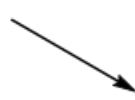

BTz-Aggregate-2

$+18.4 \mathrm{kcal} / \mathrm{mol}$

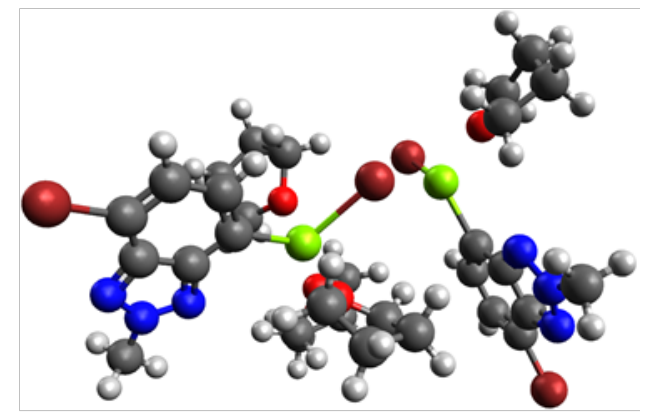

BTz-aggregate-2

Figure S20: (a) Calculated benzotriazole Grignard aggregation structures and their energy relative to Grignard monomer solvated with two molecules of THF. (b) Stick-and-ball visualized benzotriazole aggregates. 


\section{Fluorene dimer monomer coordination structure}

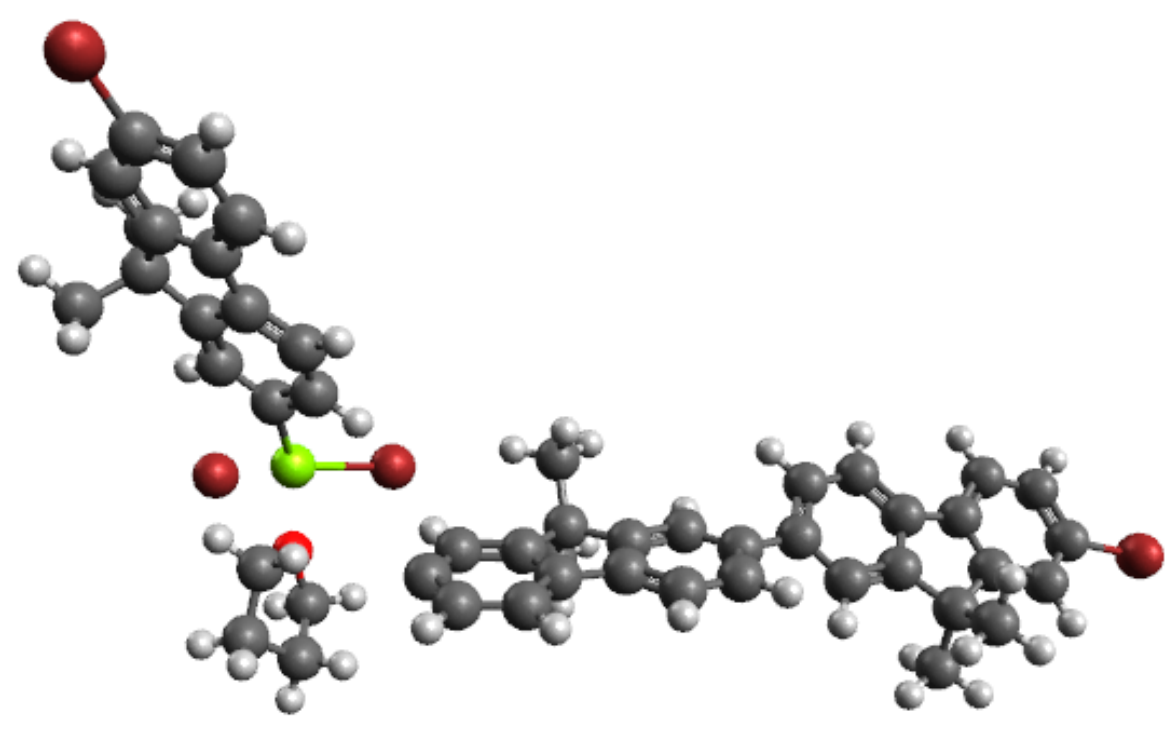

Figure S21: Chain-end bromide abstraction product of the geometry-optimized complex between fluorene monomer and the fluorene dimer radical anion. 


\section{EPR data}

All polymer samples were made the day of measurement to minimize any potential for oxidative doping via oxidation under ambient atmosphere.

Reaction mixture preparation for EPR

In a nitrogen filled glovebox, 4,7-dibromo-2-(2-octyldodecyl)-2H-benzo[d][1,2,3]triazole (0.18 $\mathrm{mmol})$ and lithium chloride $(1.44 \mathrm{mmol}, 8 \mathrm{eq})$ was charged into a vial with stir bar. THF was then charged into the vial the solution set to stir before $\mathrm{iPrMgBr}(256 \mu \mathrm{L}, 0.75 \mathrm{M}, 1 \mathrm{eq})$ was injected. The vial was wrapped in foil and set to stir for 1 hour. The foil was then removed and the vial was placed under irradiation with white LEDs for 24 hours. 200 microliters of the solution were charged into a quartz tube $(2.4 \mathrm{~mm}$ o.d. and $2 \mathrm{~mm}$ i.d.) and the tube sealed with a UV-cure epoxy. The tube was removed from the glovebox for EPR measurement. While a signal was seen by EPR at typical reaction concentrations $(50 \mathrm{mM}),{ }^{1}$ the higher concentration $(500 \mathrm{mM})$ was used to improve the signal-to-noise ratio of the measurement. Lower equivalents of $\mathrm{LiCl}$ were used at these concentrations as saturation at these concentrations required fewer equivalents.

\section{Preparation of sodium naphthalide doped P1 for EPR}

In a nitrogen filled glovebox, a sample of $5.0 \mathrm{mg}$ of $\mathbf{P} 1\left(\mathrm{M}_{\mathrm{n}}: 11.7 \mathrm{~kg} / \mathrm{mol}\right.$, Đ 1.52) prepared according to literature procedures ${ }^{1}$ was dissolved in $200 \mu \mathrm{L}$ of THF and charged into a quartz tube (2.4 mm o.d. and $2 \mathrm{~mm}$ i.d.). Sodium naphthalide THF solution ( $8.9 \mathrm{mM}, 1 \mathrm{eq}$ ) was then charged into the tube. The tube was then sealed with a UV-cure epoxy and the solution was given 1 hour to mix through diffusion. The tube was removed from the glovebox for EPR measurement.

\section{Preparation of 1-MgBr for EPR}

In a nitrogen filled glovebox, 4,7-dibromo-2-(2-octyldodecyl)-2H-benzo[d][1,2,3]triazole (82 $\mu$ mol) and lithium chloride $(6.6 \mathrm{mmol}, 8 \mathrm{eq})$ was charged into a vial with stir bar. THF was then charged into the vial the solution set to stir before $\mathrm{iPrMgBr}(117 \mu \mathrm{L}, 0.75 \mathrm{M}, 1 \mathrm{eq})$ was injected. The vial was wrapped in foil and set to stir for 1 hour. After the hour, 200 microliters of the solution were charged into a quartz tube $(2.4 \mathrm{~mm}$ o.d. and $2 \mathrm{~mm}$ i.d.) and the tube sealed with a UV-cure epoxy. The tube was removed from the glovebox for EPR measurement. Higher concentrations (500 $\mathrm{mM}$ ) were used than typical reaction concentrations $(50 \mathrm{mM}),{ }^{1}$ to improve the signal-to-noise ratio of the measurement. Lower equivalents of $\mathrm{LiCl}$ were used at these concentrations as saturation at these concentrations required fewer equivalents.

\section{Preparation of neutral P1 for EPR}

P1 was prepared according to literature procedures ${ }^{1}$ before being isolated $\left(M_{n} 13.8 \mathrm{~kg} / \mathrm{mol}\right.$, $Đ$ 1.56) and moved into a nitrogen filled glovebox. P1 $(6.0 \mathrm{mg}, 1.7 \mu \mathrm{mol})$ was dissolved into 200 microliters of THF before being charged into a quartz tube $(2.4 \mathrm{~mm}$ o.d. and $2 \mathrm{~mm}$ i.d) and the tube sealed with a UV-cure epoxy. The tube was removed from the glovebox for EPR measurement. This sample concentration was the limit of the polymer sample in THF at room temperature. 


\section{CW-EPR line fitting}

The CW-EPR data were fit using EasySpin ${ }^{13}$, which can show whether the line shape is Gaussian or Lorentzian. Without hyperfine structure, we cannot gain detailed insight into the spin microenvironment. There can be several reasons for line broadening. When the line shape is Gaussian, broadening is typically due to inhomogeneous broadening, which can come from unresolved hyperfine structure or dipolar interactions. When the line shape is Lorentzian, the broadening is typically due to homogeneous broadening, which can be due to spin-spin interactions, motion of the radical, fluctuations in the local field of the unpaired electron, or hindered rotation. ${ }^{14}$ The fit for the doped $\mathbf{P} 1$ signal is Gaussian, and the fit for the reaction mixture signal was Lorentzian (see below). The environment of the radical anion is different in the reaction mixture than when it is independently generated from isolated P1 with sodium naphthalenide, so these differences are not unexpected.

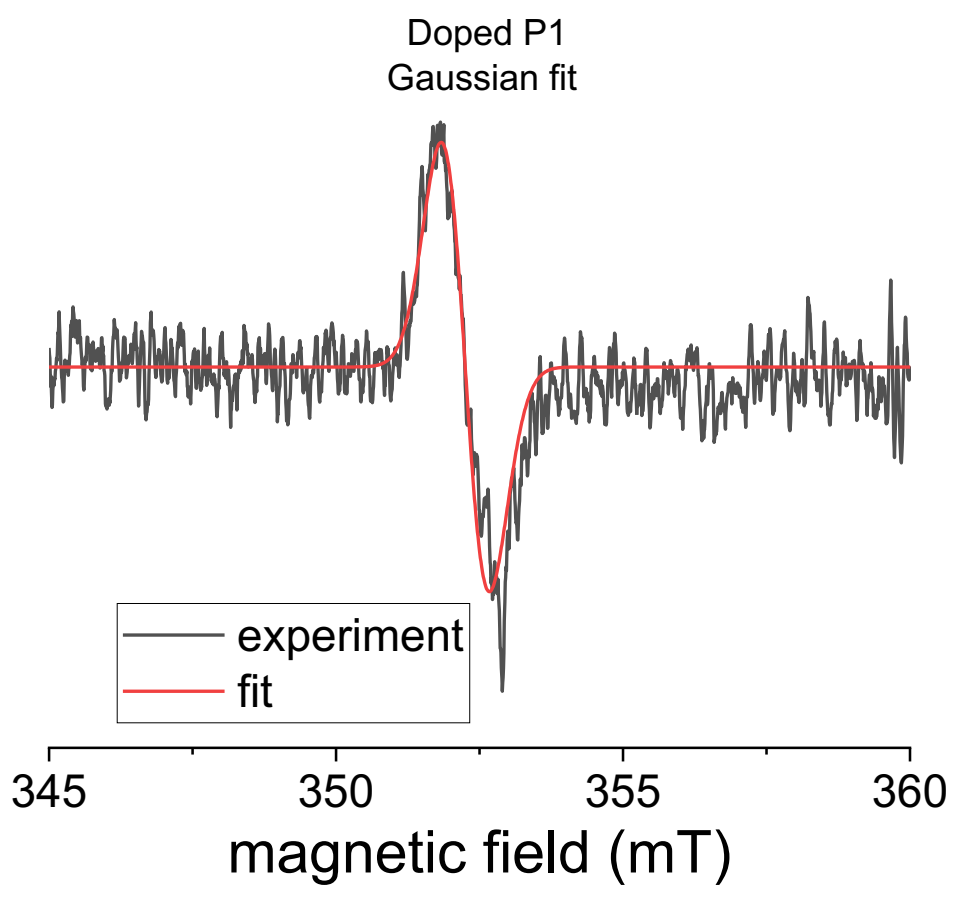

Figure S22. Fitted CW-EPR signal of doped P1. 


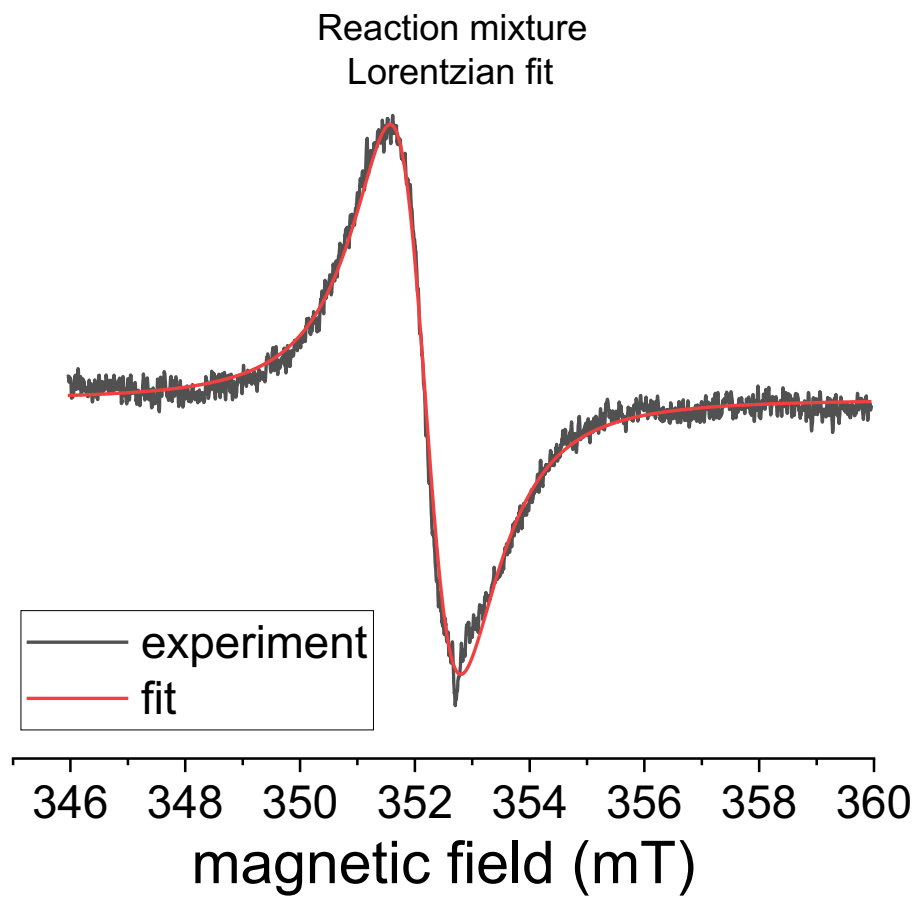

Figure S23. Fitted CW-EPR data of $\mathbf{P 1}$ reaction mixture. 


\section{Cartesian coordinates of calculated structures with energies and frequency analysis}

Energies are reported in hartrees.

Benzotriazole dimer radical anion

Electronic energy calculated $(E)=-6016.308636$

Standard state Gibbs free energy $\left(G^{\circ}\right)=-6016.120812$

Number of imaginary frequencies (Nimag) $=0$

C $-2.45817-1.24658-0.21646$

C $-1.08996-1.04522-0.03535$

C -0.489270 .226360 .14869$

C -1.443391 .313410 .13270$

C $-2.843761 .10019-0.05299$

C $-3.35782-0.19849-0.23130$

Br $-5.22559-0.47331-0.48011$

$\mathrm{N}-3.466752 .28260-0.02770$

$\mathrm{N}-1.268012 .636070 .26505$

$\mathrm{N}-2.480103 .149510 .15193$

C -2.733334 .560180 .34336$

C 0.918360 .408850 .33361

C $1.87274-0.677970 .34393$

C 1.518281 .679170 .52847

C 2.886441 .879870 .71112

C 3.786760 .832320 .71681

C $3.27290-0.465590 .53182$

N $1.69931-1.998430 .18955$

N $3.89773-1.646360 .48682$

N $2.91488-2.509540 .27103$

Br 5.654881 .106470 .96354

C $3.14512-3.936940 .25668$

H - $2.81893-2.26062-0.34896$

H - $0.45694-1.91786-0.03694$

$\mathrm{H}-1.846775 .112210 .04331$

H - 2.959484 .768541 .39060

H -3.57912 4.84715 -0.27648

H 0.884822 .551360 .53761

H 3.246452 .892980 .85242

H $4.13334-4.12233-0.15630$

H $2.38648-4.40263-0.36668$

H 3.08886 -4.34266 1.26841

Benzotriazole internal nitrogen monomer coordination dimer radical anion (5) 
Electronic energy calculated $(E)=-12031.29218294$

Standard state Gibbs free energy $\left(G^{\circ}\right)=-12030.8913$

Number of imaginary frequencies $(\mathrm{Nimag})=0$

C $-3.26082-1.01143-1.17280$

C $-1.91829-0.90965-0.72722$

C $-1.351810 .25553-0.25406$

C $-2.239071 .37527-0.23419$

C $-3.579881 .28667-0.68261$

C -4.09437 0.06686-1.16708

$\mathrm{Br}-5.89347-0.04460-1.75805$

$\mathrm{N}-4.162362 .48433-0.52485$

$\mathrm{N}-2.045602 .637580 .19330$

$\mathrm{N}-3.202763 .22115-0.01140$

C -3.434114.608310.35466

C 0.058600 .343300 .14166

C $0.77646-0.749360 .67225$

C $0.799811 .51985-0.10663$

C 2.164951 .611760 .11092

C 2.873320 .516440 .59363

C $2.19525-0.671730 .87667$

N $0.37989-1.976321 .08580$

N 2.65314 -1.83330 1.34995

N $1.54322-2.588721 .54668$

Br 4.754370 .616270 .87476

C $1.67708-4.016751 .33743$

H -3.61383 - $1.97271-1.52429$

$\mathrm{H}-1.30853-1.80035-0.79447$

H -2.46838 5.079110 .50694

H -4.021824.65089 1.27062

H -3.97359 $5.09630-0.45278$

H $0.285702 .38261-0.50645$

H $2.683942 .53607-0.11131$

H $2.57313-4.348921 .85631$

H $1.76249-4.248140 .27031$

H $0.81647-4.532331 .75869$

C $-4.27551-5.80716-0.61790$

C -3.01047 -5.47600-1.00099

C $-2.22354-4.56373-0.23197$

C -2.63912 -3.94547 0.93089

C $-3.96087-4.322851 .29255$

C $-4.77955-5.221400 .55971$

$\mathrm{N}-5.95877-5.350481 .18583$

$\mathrm{N}-4.68688-3.926632 .36297$

$\mathrm{N}-5.82451-4.564212 .23525$

C -6.91955 -4.36335 3.16705

$\mathrm{Br}-5.34885-7.02052-1.62041$ 
Mg -1.38874 -2.62508 2.07543

C -3.813560 .475883 .81794$

C $-3.63185-0.556862 .72034$

O $-2.21502-0.881522 .74720$

C -1.486760 .078903 .56012$

C -2.471271 .207863 .80707$

H -3.97228 -0.01511 4.78072

H -4.662901 .130663 .62238$

H -3.86090 - 0.152251 .73357

H -4.18898 -1.47944 2.86991

H -0.598690 .378363 .00593$

H - $1.19086-0.422334 .48263$

H -2.43142 1.932242 .99039

H -2.26016 1.73117 4.73946

H - $2.58869-5.90847-1.90125$

H - $1.22859-4.36347-0.62457$

H - $7.50742-3.497652 .86291$

H -6.50402 -4.19989 4.15724

H -7.54011 -5.25436 3.15774

Br -0.69594 -3.60696 4.32255

Benzotriazole meta carbon monomer coordination dimer radical anion (6)

Electronic energy calculated $(E)=-12031.2792$

Standard state Gibbs free energy $\left(\mathrm{G}^{\circ}\right)=-12030.87851$

Number of imaginary frequencies (Nimag) $=0$

C $-2.76482-0.17421-1.26346$

C $-1.52266-0.54088-0.69780$

C $-0.592650 .40937-0.15565$

C $-1.124061 .75256-0.10117$

C $-2.399502 .09306-0.64038$

C $-3.221131 .11675-1.25467$

Br $-4.910631 .59807-1.97316$

N $-2.608233 .39522-0.45986$

$\mathrm{N}-0.596442 .879150 .39356$

$\mathrm{N}-1.503573 .809030 .14614$

C -1.358015 .166050 .62496$

C 0.729650 .061900 .25607

C $1.26167-1.276760 .16526$

C 1.649250 .989640 .79430

C 2.946230 .657371 .20519

C $3.43604-0.625921 .11861$

C $2.57837-1.611240 .58853$

$\mathrm{N} 0.69465-2.41006-0.27395$

N $2.77053-2.921900 .39875$

N $1.61946-3.33259-0.10396$ 
Br 5.19119-1.07959 1.68821

C 1.41559 -4.70071 -0.53549

H -3.36702 -0.95208 - 1.72043

$\mathrm{H}-1.16966-1.54251-0.90273$

H -0.30361 5.428980 .60567

$\mathrm{H}-1.740705 .253051 .64314$

H - $1.918895 .82302-0.03433$

H 1.344512 .018650 .89866

H 3.577091 .443511 .60416

H $1.96549-5.358800 .13191$

H $1.77314-4.82894-1.55766$

H $0.35169-4.91645-0.48659$

C -3.51664 -6.33077 0.33419

C $-3.13347-5.47921-0.65848$

C $-2.85706-4.10403-0.38426$

C - $2.94644-3.513760 .86018$

C $-3.35134-4.437361 .86183$

C -3.63353 -5.80997 1.63745

$\mathrm{N}-3.97441-6.383232 .80139$

$\mathrm{N}-3.53227-4.217443 .18423$

$\mathrm{N}-3.88670-5.386293 .65931$

C $-4.24690-5.564945 .05442$

$\mathrm{Br}-3.88841-8.16897-0.00046$

$\mathrm{Mg}-2.52955-1.455761 .30519$

C $0.11725-0.736794 .67253$

C $-0.73835-0.199523 .53833$

O - $1.07817-1.367622 .73796$

C $-0.30720-2.521453 .17196$

C $0.80194-1.942234 .02938$

H -0.51036 - 1.054865 .50817

H 0.820440 .012045 .03686

H -0.186790 .488342 .89675$

H - 1.668330 .263553 .86056

H $0.03704-3.042382 .28120$

H -0.97201 -3.17458 3.73886

H $1.63831-1.619203 .40571$

H $1.17230-2.665524 .75588$

H -3.03482 -5.84477-1.67433

H $-2.55138-3.51310-1.24466$

H -5.31022 -5.36745 5.18736

H -3.66457 -4.86948 5.65163

H -4.02227 -6.58945 5.33632

$\mathrm{Br}-4.294320 .030062 .27574$

Benzotriazole chain-end nitrogen monomer coordination dimer radical anion (4) 
Electronic energy calculated $(E)=-12031.29378$

Standard state Gibbs free energy $\left(G^{\circ}\right)=-12030.89339$

Number of imaginary frequencies (Nimag) $=0$

C $-2.07302-1.347460 .30722$

C $-0.73386-1.26686-0.03378$

C $0.07526-0.145880 .24925$

C -0.587810 .925670 .92648$

C -1.969200 .826781 .31301$

C - $2.70409-0.302760 .98432$

Br $-4.56349-0.363851 .37982$

$\mathrm{N}-2.314201 .958621 .96630$

$\mathrm{N}-0.135552 .118071 .31442$

$\mathrm{N}-1.146572 .703941 .99495$

C - 1.249494 .147771 .88698

C $1.48244-0.08581-0.13680$

C $2.20329-1.19290-0.70753$

C 2.255241 .058450 .01810

C $3.620481 .15360-0.34325$

C $4.292720 .09805-0.88277$

C $3.57299-1.09869-1.06884$

N $1.81479-2.45027-0.99348$

N $3.97263-2.28026-1.55772$

N 2.89134 -3.02192 -1.47782

Br $6.123640 .20379-1.37183$

C $2.86759-4.38663-1.97296$

$\mathrm{H}-2.63952-2.231270 .04014$

H $-0.29634-2.10837-0.54713$

H -0.260454 .567232 .05538$

$\mathrm{H}-1.926224 .520782 .65078$

H - 1.606474 .434790 .89262

H 1.798031 .938350 .44129

H $4.130302 .09578-0.18176$

H $3.87150-4.79271-1.89398$

H $2.54277-4.39424-3.01298$

H $2.17560-4.96094-1.36387$

C -8.28541 4.28943 1.94807

C -7.140825.00720 2.11979

C -5.93249 4.37210 2.54267

C -5.79207 3.02267 2.80605

C -7.01284 2.31477 2.61927

C -8.23654 2.90564 2.20508

$\mathrm{N}-9.181161 .956252 .13232$

$\mathrm{N}-7.263850 .995902 .78962$

$\mathrm{N}-8.531580 .865362 .48532$

C -9.21597 -0.40925 2.60420

$\mathrm{Br}-9.913715 .102931 .38555$ 
Mg -3.86592 2.26396 3.39911

C - $2.89737-1.055806 .00141$

C - $2.52796-0.113364 .86730$

O -3.765450 .559294 .51796$

C $-4.88789-0.039085 .21554$

C $-4.36241-1.375805 .70314$

H - $2.80788-0.547876 .96424$

H -2.25810 -1.93833 6.01859

H - $2.18038-0.649393 .98248$

$\mathrm{H}-1.797460 .645545 .14411$

H -5.71646 -0.09962 4.51290

H -5.159200 .617336 .04610$

$\mathrm{H}-4.43880-2.126264 .91281$

H $-4.90913-1.735906 .57472$

H - 7.139156 .075511 .93557

H -5.07933 5.03664 2.66098

H $-9.58708-0.534643 .62114$

H $-8.51225-1.201782 .36673$

H - $10.04611-0.416011 .90407$

Br -2.91046 3.81667 5.21004

Benzotriazole constrained geometry optimization of ground state ortho monomer coordination dimer radical anion (7)

Electronic energy calculated $(E)=-12031.27277$

Standard state Gibbs free energy $\left(\mathrm{G}^{\circ}\right)=-12030.87348$

Number of imaginary frequencies (Nimag) $=1$

C $-2.772340 .87426-1.30947$

C $-1.672110 .10876-0.91647$

C $-0.473460 .65046-0.39259$

C $-0.476642 .09310-0.30834$

C $-1.593312 .88252-0.73274$

C $-2.760512 .27435-1.23535$

Br -4.12915 3.32517-2.03534

N - $1.298494 .17191-0.56339$

N 0.464552 .941890 .11014

N -0.06027 4.14864 -0.07431

C 0.605905 .347060 .37731

C $0.62282-0.171270 .03805$

C $0.67388-1.60214-0.14800$

C 1.750150 .342110 .70008

C $2.81654-0.444831 .16806$

C $2.84115-1.806041 .00536$

C $1.75293-2.395080 .33082$

$\mathrm{N}-0.17472-2.43811-0.76994$

N $1.52572-3.673760 .00325$ 
N $0.37881-3.62336-0.64885$

Br 4.27827 -2.87757 1.63838

C $-0.26497-4.82213-1.15320$

H -3.61231 $0.37104-1.77861$

H - $1.75910-0.96177-1.01385$

H 1.677155 .226230 .23455

H 0.396765 .531091 .43334

H $0.244976 .18321-0.21622$

H 1.806131 .404500 .87592

H 3.635780 .049221 .67774

H 0.50644 -5.55746 -1.36278

H $-0.80028-4.56681-2.06362$

H $-0.96357-5.21230-0.41277$

C $-4.75241-4.45137-0.16129$

C $-5.55429-3.48957-0.69896$

C $-5.38317-2.11102-0.36210$

C -4.43433 -1.62547 0.51599

C -3.62896 -2.66447 1.05772

C -3.75109-4.04146 0.74007

$\mathrm{N}-2.81343-4.729261 .40954$

$\mathrm{N}-2.60401-2.558011 .93188$

$\mathrm{N}-2.18378-3.786912 .08726$

C - $1.03198-4.102872 .91431$

$\mathrm{Br}-4.95192-6.29339-0.60322$

$\mathrm{Mg}-3.918430 .419790 .92696$

C -6.96565 3.523370 .78072

C -5.64651 2.98544 1.30236

O -5.548401 .637810 .75543$

C -6.819001 .234020 .17805$

C -7.81966 2.26071 0.67566

H $-6.830193 .97687-0.20319$

$\mathrm{H}-7.388094 .272201 .45061$

H -5.63213 2.90370 2.38909

$\mathrm{H}-4.774333 .543760 .97065$

H -7.02768 0.217850.50582

$\mathrm{H}-6.716011 .25638-0.90866$

H -8.20253 1.973551 .65758

H -8.66407 2.36662-0.00526

H $-6.33284-3.76738-1.40040$

H $-6.06170-1.42758-0.86653$

$\mathrm{H}-0.90795-3.304863 .63953$

$\mathrm{H}-0.14034-4.180102 .29188$

$\mathrm{H}-1.21410-5.049603 .41535$

Br -2.733211 .454602 .83961$

Benzotriazole excited state geometry ortho monomer coordination dimer radical anion 
Electronic energy calculated $(E)=-12031.26477$

Standard state Gibbs free energy $\left(G^{\circ}\right)=-12030.78572$

Number of imaginary frequencies (Nimag) $=0$

C $-2.798250 .83976-1.43344$

C $-1.690620 .08345-0.94903$

C $-0.551540 .63960-0.39458$

C $-0.564802 .07105-0.32277$

C $-1.657642 .84654-0.81906$

C - $2.767202 .22013-1.40042$

Br $-4.150663 .25646-2.19176$

$\mathrm{N}-1.400934 .14713-0.62026$

$\mathrm{N} 0.341662 .929170 .16055$

$\mathrm{N}-0.198514 .12189-0.05273$

C 0.407055 .318810 .46698

C $0.56886-0.199150 .08944$

C $0.61316-1.60632-0.13079$

C 1.627740 .327260 .82581

C $2.67286-0.446591 .33919$

C $2.69894-1.815291 .13388$

C $1.67202-2.411640 .39498$

$\mathrm{N}-0.21361-2.41559-0.81067$

$\mathrm{N} 1.47023-3.692070 .04791$

N $0.35842-3.62568-0.70131$

Br 4.10646 -2.88097 1.84582

C $-0.30895-4.81177-1.16040$

$\mathrm{H}-3.600990 .31864-1.94057$

$\mathrm{H}-1.74737-0.99080-1.04635$

H 1.486315 .184770 .46904

H 0.062585 .511721 .48692

H $0.126996 .15206-0.17405$

H 1.643121 .388511 .02444

H 3.462860 .030251 .90564

H $0.44569-5.57271-1.35072$

H $-0.84279-4.57412-2.07917$

$\mathrm{H}-1.02705-5.18247-0.42052$

C -4.79063 -4.46164-0.08821

C $-5.62114-3.50806-0.60407$

C -5.43305 -2.12467 - 0.31692

C - $4.43379-1.622160 .49472$

C $-3.59658-2.649191 .00421$

C $-3.73273-4.036230 .73565$

$\mathrm{N}-2.74723-4.699771 .35923$

$\mathrm{N}-2.51746-2.507001 .79939$

$\mathrm{N}-2.08085-3.729731 .96596$

C $-0.86353-4.004862 .70202$

$\mathrm{Br}-5.03060-6.31076-0.47981$ 
Mg -3.76297 0.35380 0.96032

C -6.79937 3.53347 0.77427

C -5.460712 .995751 .25312$

O -5.402811 .633510 .75053$

C -6.675561 .238390 .20201$

C -7.662402 .273540 .71523$

$\mathrm{H}-6.697703 .97301-0.22020$

H -7.19529 4.29593 1.44643

H -5.38388 2.94895 2.33870

H -4.599463 .534810 .86566$

H -6.880450 .222100 .53241$

H -6.59944 $1.25639-0.88847$

H -8.013572 .000671 .71371$

H -8.53210 2.37256 0.06410

H -6.43892 -3.80849 - 1.25003

H -6.13804-1.45124 -0.80059

$\mathrm{H}-0.67180-3.156523 .35227$

H - $0.02646-4.132152 .01227$

H - $1.00785-4.914343 .28113$

Br -2.71318 1.53813 2.81658

Benzotriazole TS from ortho to ipso monomer coordination dimer radical anion (TS-1)

Electronic energy calculated $(E)=-12031.26733$

Standard state Gibbs free energy $\left(G^{\circ}\right)=-12030.86354$

Number of imaginary frequencies (Nimag) $=1$

C $-1.220300 .10920-1.63989$

C $0.157670 .17124-1.80907$

C $0.901851 .37939-1.82173$

C $0.091392 .55981-1.65122$

C $-1.318962 .49914-1.44222$

C $-1.995131 .25368-1.37236$

$\mathrm{Br}-3.898201 .20546-1.58400$

$\mathrm{N}-1.792093 .74007-1.34748$

N $0.429353 .85166-1.67110$

$\mathrm{N}-0.712344 .49971-1.50113$

C $-0.755315 .93561-1.36275$

C $2.324551 .42348-1.98637$

C $3.132690 .25416-2.22172$

C $3.072772 .61136-1.91708$

C $4.469892 .66891-2.04907$

C $5.227581 .54472-2.25500$

C $4.547320 .31250-2.34435$

N $2.78133-1.03211-2.38043$

N $5.01006-0.92514-2.56253$

N $3.91625-1.66204-2.57606$ 
Br $7.117811 .62318-2.43325$

C $3.95043-3.09692-2.77793$

$\mathrm{H}-1.71122-0.85254-1.73579$

H $0.67766-0.76156-1.95711$

H $0.015306 .36713-1.99698$

H - $0.580136 .22478-0.32491$

H - $1.736366 .27831-1.67987$

H $2.555543 .54125-1.74479$

H $4.952493 .63707-1.97889$

H $4.94218-3.35925-3.13165$

H $3.20211-3.36355-3.52097$

H $3.73728-3.61126-1.84129$

C $-0.37146-5.045361 .04557$

C $-1.49776-4.622790 .40058$

C - $1.86309-3.239850 .35076$

C - $1.14692-2.219690 .94694$

C - $0.00806-2.716991 .62585$

C $0.41597-4.069421 .68523$

N $1.55237-4.136572 .39888$

N $0.88918-1.995742 .32494$

N $1.75588-2.877732 .74054$

C $2.91419-2.445383 .49384$

Br $0.13081-6.884441 .09215$

$\mathrm{Mg}-1.28210-0.049420 .95089$

C -5.022531 .526262 .51727$

C -3.532451 .671812 .27130$

O -3.105630 .370551 .77891$

C $-4.16717-0.608141 .93881$

C -5.158640 .047692 .88254$

H -5.579091 .744991 .60351$

H -5.370832 .199313 .30159$

$\mathrm{H}-2.972491 .883043 .18368$

H -3.274502 .417461 .52168$

H $-3.72145-1.525412 .31801$

$\mathrm{H}-4.60374-0.793970 .95509$

H $-4.87769-0.130473 .92451$

H $-6.16783-0.337392 .73801$

H $-2.12734-5.35259-0.09519$

H $-2.76798-3.01896-0.21040$

H $2.58161-1.687234 .19884$

H $3.64438-2.016232 .80821$

H $3.32702-3.304704 .01103$

Br 0.016991 .761562 .08576

Benzotriazole ipso monomer coordination dimer radical anion (8)

Electronic energy calculated $(E)=-12031.27903$ 
Standard state Gibbs free energy $\left(G^{\circ}\right)=-12030.87732$ Number of imaginary frequencies (Nimag) $=0$

\begin{tabular}{llll}
$\mathrm{C}-2.60365$ & -0.52134 & -0.98470 \\
$\mathrm{C}-1.25883$ & -0.56196 & -0.69902 \\
$\mathrm{C}-0.54232$ & 0.47867 & -0.05322 \\
$\mathrm{C}-1.35815$ & 1.63010 & 0.25259 \\
$\mathrm{C}-2.75237$ & 1.66898 & -0.02253 \\
$\mathrm{C}-3.47253$ & 0.52917 & -0.54073 \\
$\mathrm{Br}-4.93249$ & 0.98947 & -1.82804 \\
$\mathrm{~N}-3.21790$ & 2.86377 & 0.32952 \\
$\mathrm{~N}-1.02520$ & 2.81833 & 0.77496 \\
$\mathrm{~N}-2.15139$ & 3.50296 & 0.78610 \\
$\mathrm{C}-2.23431$ & 4.83538 & 1.34482 \\
$\mathrm{C}$ & 0.86774 & 0.39198 & 0.23695 \\
$\mathrm{C} 1.69276$ & -0.74148 & -0.10331 \\
$\mathrm{C} 1.56859$ & 1.41200 & 0.88711 \\
$\mathrm{C} 2.94397$ & 1.36136 & 1.19391 \\
$\mathrm{C} 3.71362$ & 0.28042 & 0.86733 \\
$\mathrm{C} 3.07736$ & -0.78977 & 0.20598 \\
$\mathrm{~N} 1.39458$ & -1.89815 & -0.72102 \\
$\mathrm{~N} 3.57460$ & -1.95619 & -0.22451 \\
$\mathrm{~N} 2.53022$ & -2.55510 & -0.75204 \\
$\mathrm{~B} r$ & 5.56488 & 0.19628 & 1.27281 \\
$\mathrm{C} 2.64000$ & -3.84824 & -1.40239 \\
$\mathrm{H}-3.03863$ & -1.35780 & -1.51946 \\
$\mathrm{H}-0.71960$ & -1.45011 & -0.99010 \\
$\mathrm{H}-1.30108$ & 5.35487 & 1.14327 \\
$\mathrm{H}-2.40245$ & 4.78612 & 2.42149 \\
$\mathrm{H}-3.06084$ & 5.35429 & 0.86716 \\
$\mathrm{H} 1.03439$ & 2.30221 & 1.17835 \\
$\mathrm{H} 3.38941$ & 2.20773 & 1.70292 \\
$\mathrm{H} 3.50442$ & -4.35966 & -0.99015 \\
$\mathrm{H} 2.76436$ & -3.71071 & -2.47613 \\
$\mathrm{H} 1.73377$ & -4.41354 & -1.20381 \\
$\mathrm{C}-6.99378$ & -4.86366 & -0.04155 \\
$\mathrm{C}-6.17548$ & -4.24997 & -0.94151 \\
$\mathrm{C}-5.52396$ & -3.01734 & -0.62583 \\
$\mathrm{C}-5.64286$ & -2.34266 & 0.57291 \\
$\mathrm{C}-6.51049$ & -3.01723 & 1.47637 \\
$\mathrm{C}-7.18041$ & -4.23941 & 1.20684 \\
$\mathrm{~N}-7.91393$ & -4.58284 & 2.27595 \\
$\mathrm{~N}-6.87160$ & -2.64819 & 2.72680 \\
$\mathrm{~N}-7.67676$ & -3.60221 & 3.12468 \\
$\mathrm{C}-8.33625$ & -3.55200 & 4.41712 \\
\hline & -6.50772 & -0.43317 \\
\hline & -0.50065 & 1.13581
\end{tabular}


$\begin{array}{lll}\text { C }-7.22811 & 2.81221 & 2.30484 \\ \text { C }-5.92644 & 2.03705 & 2.40043 \\ \text { O }-6.14990 & 0.83734 & 1.61046 \\ \text { C }-7.55990 & 0.68967 & 1.29788 \\ \text { C }-8.26511 & 1.69576 & 2.19008 \\ \text { H }-7.23653 & 3.43455 & 1.40683 \\ \text { H }-7.38215 & 3.45616 & 3.17082 \\ \text { H }-5.70402 & 1.72812 & 3.42267 \\ \text { H }-5.06836 & 2.55479 & 1.97729 \\ \text { H }-7.83700 & -0.34487 & 1.48803 \\ \text { H }-7.69309 & 0.91630 & 0.23842 \\ \text { H }-8.46701 & 1.25991 & 3.17141 \\ \text { H }-9.21050 & 2.02697 & 1.76021 \\ \text { H }-6.01095 & -4.69882 & -1.91455 \\ \text { H }-4.90030 & -2.61035 & -1.41732 \\ \text { H }-9.23386 & -2.93789 & 4.34886 \\ \text { H }-7.64862 & -3.12314 & 5.14059 \\ \text { H }-8.60165 & -4.56606 & 4.70017 \\ \text { Br }-3.23439 & -0.44783 & 3.20613\end{array}$

Benzotriazole dimer TS from ipso monomer coordination to magnesium-ion-radical cage (TS-2)

Electronic energy calculated $(E)=-12031.26993$

Standard state Gibbs free energy $\left(\mathrm{G}^{\circ}\right)=-12030.87073$

Number of imaginary frequencies (Nimag) $=1$

C $0.74748-0.19244-1.46252$

C $2.12609-0.26910-1.22128$

C $2.895290 .78068-0.73121$

C $2.155761 .98484-0.48058$

C $0.754322 .03977-0.67433$

C $-0.015150 .91058-1.08107$

$\mathrm{Br}-1.565651 .54902-2.68153$

N $0.338983 .27682-0.37777$

N $2.553123 .19982-0.06655$

$\mathrm{N} 1.441403 .90536-0.04095$

C 1.436395 .292830 .38355

C $4.353540 .62907-0.62930$

C $5.07362-0.60465-0.75522$

C $5.138611 .71004-0.27132$

C $6.534791 .63261-0.04091$

C $7.209490 .45470-0.15880$

C $6.46872-0.68749-0.52362$

N $4.66091-1.84079-1.09267$

N $6.86228-1.95452-0.71808$

N $5.74964-2.56890-1.04397$

Br 9.07581 0.328260.14655 
C $5.72539-3.97867-1.39761$

H $0.26901-1.06339-1.89907$

H $2.60196-1.21911-1.40675$

H 2.371825 .748950 .07137

H 1.339385 .348281 .46754

H $0.596445 .79232-0.09103$

H $4.676102 .67961-0.16532$

H 7.06304 2.53700 0.23509

H $6.56929-4.46446-0.91764$

H $5.79681-4.08509-2.47945$

H $4.78979-4.40335-1.04498$

C $-3.44524-4.44819-0.82866$

C - $2.77316-3.73269-1.77503$

C $-2.25897-2.42971-1.49188$

C - $2.37269-1.78952-0.27439$

C $-3.08098-2.570750 .67731$

C $-3.61892-3.862140 .44052$

N $-4.22344-4.296511 .55709$

$\mathrm{N}-3.38176-2.255411 .95784$

$\mathrm{N}-4.03583-3.299242 .40097$

C $-4.60028-3.330693 .73863$

Br -4.13775 -6.18799-1.18651

Mg -1.62761 0.13549 0.30900

C -4.568373 .194891 .32508$

C -3.289382 .387441 .55405$

O -3.323701 .315700 .54911$

C $-4.611861 .26974-0.16059$

C -5.535532 .139440 .70810$

H -4.380604 .024750 .61618$

H -4.94269 3.61186 2.27641

H -3.26369 1.913552 .54811

H -2.371972 .973221 .39484$

H $-4.937370 .21602-0.24117$

H $-4.465421 .69258-1.17305$

H -6.031521 .536961 .49374$

H -6.307692 .615240 .09009$

H $-2.61765-4.15684-2.76058$

H $-1.74941-1.93925-2.31875$

H $-5.57816-2.849073 .73662$

H $-3.92946-2.800034 .40886$

H $-4.69976-4.369304 .04046$

Br -0.254100 .300562 .47692$

Benzotriazole magnesium-ion-radical cage dimer complex (9)

Electronic energy calculated $(E)=-12031.29111$

Standard state Gibbs free energy $\left(G^{\circ}\right)=-12030.88534$ 
Number of imaginary frequencies (Nimag) $=0$

C - $2.28747-0.575271 .25849$

C $-0.91460-0.851400 .97647$

C 0.047330 .127090 .88426

C -0.407141 .464931 .08839$

C -1.771151 .760931 .36292$

C -2.679730 .702741 .43436$

Br -5.37997 2.44719-0.20153

$\mathrm{N}-1.895323 .092001 .48525$

N 0.256192 .633551 .04960

$\mathrm{N}-0.676513 .529641 .28626$

C -0.354644 .942531 .38961$

C $1.46260-0.187150 .60683$

C $1.85862-1.30889-0.17834$

C 2.498610 .577101 .09219

C 3.866980 .284770 .85135

C $4.23896-0.791700 .10361$

C $3.21957-1.60526-0.42877$

N $1.12418-2.23955-0.81522$

N $3.28184-2.69922-1.20217$

N $2.01972-3.01230-1.38155$

Br 6.05876 - $1.20063-0.23199$

C $1.62765-4.12892-2.22553$

H -2.98968 - 1.397641 .31350

H - $0.63518-1.885830 .83077$

Н 0.449745 .162370 .69379

H -0.04113 5.17149 2.40745

H - 1.244475 .510801 .13713

H 2.275771 .450411 .68846

H 4.619140 .936301 .27801

H $2.46039-4.82353-2.27102$

H $1.38863-3.76275-3.22304$

H $0.75595-4.60433-1.78524$

C $-6.07873-3.948160 .51448$

C -5.70957 -2.99030 - 0.38214

C -5.57216-1.62422 0.01396

C -5.78726-1.14640 1.29113

C -6.17671 -2.17712 2.18887

C $-6.32582-3.546031 .84124$

$\mathrm{N}-6.70028-4.236942 .92842$

$\mathrm{N}-6.47589-2.079903 .50480$

$\mathrm{N}-6.76234-3.307603 .86184$

C -7.20064 -3.62632 5.20861

Br -6.26738 -5.77857 0.01649

$\mathrm{Mg}-5.644610 .937161 .82745$

C -9.94122 1.60907 1.78063 


$$
\begin{array}{lll}
\text { C }-8.70581 & 0.78236 & 1.47101 \\
\text { O }-7.63087 & 1.42733 & 2.20035 \\
\text { C }-8.05251 & 2.73558 & 2.66572 \\
\text { C }-9.35645 & 3.01205 & 1.93836 \\
\text { H }-10.39768 & 1.27846 & 2.71674 \\
\text { H }-10.68807 & 1.53761 & 0.98982 \\
\text { H }-8.45795 & 0.80540 & 0.40763 \\
\text { H }-8.76120 & -0.25190 & 1.80454 \\
\text { H }-7.25985 & 3.44661 & 2.44048 \\
\text { H }-8.18773 & 2.67668 & 3.74707 \\
\text { H }-9.15943 & 3.45495 & 0.95938 \\
\text { H }-10.00079 & 3.68909 & 2.49950 \\
\text { H }-5.51477 & -3.26347 & -1.41322 \\
\text { H }-5.26875 & -0.93609 & -0.77156 \\
\text { H }-8.28328 & -3.52270 & 5.27802 \\
\text { H }-6.71804 & -2.93946 & 5.89767 \\
\text { H }-6.91227 & -4.65020 & 5.42793 \\
\text { Br }-4.68145 & 1.79338 & 3.99753
\end{array}
$$

Benzotriazole dimer electron delocalization transition state radical anion (TS-3)

Electronic energy calculated $(E)=-12031.29069$

Standard state Gibbs free energy $\left(G^{\circ}\right)=-12030.89213$

Number of imaginary frequencies (Nimag) $=1$

C $0.86533-0.030030 .66775$

C $2.23698-0.386240 .50313$

C $3.130100 .36911-0.22221$

C $2.599861 .55033-0.82080$

C $1.233281 .91872-0.67118$

C 0.402711 .102480 .09958

$\mathrm{Br}-2.476854 .111650 .10977$

N $1.023403 .04604-1.37076$

N $3.178602 .47304-1.60908$

N $2.198773 .30354-1.88941$

C $2.427974 .49530-2.68819$

C $4.54875-0.01182-0.36442$

C $4.98289-1.36943-0.36042$

C $5.552560 .91856-0.50483$

C $6.925170 .57728-0.62762$

C $7.33403-0.72232-0.61881$

C $6.34802-1.71994-0.48841$

N $4.28409-2.51572-0.26584$

N $6.44815-3.05710-0.46652$

N $5.20281-3.44929-0.33044$

Br $9.16023-1.20148-0.78222$

C $4.84850-4.85886-0.31856$ 
H $0.22126-0.673921 .25456$

H $2.57610-1.297200 .97706$

H $3.146364 .25539-3.46693$

H $2.817565 .28989-2.05298$

H $1.479564 .79651-3.12180$

H $5.299241 .96903-0.52588$

H $7.650681 .37479-0.72656$

H $5.72228-5.42173-0.00599$

H $4.54268-5.16412-1.31835$

H $4.02978-5.002690 .38043$

C - $2.88043-1.80170-2.53941$

C $-2.88621-0.47044-2.83262$

C $-2.701920 .51067-1.81049$

C $-2.515770 .22627-0.47234$

C $-2.51664-1.17003-0.21240$

C - $2.69135-2.17850-1.19524$

$\mathrm{N}-2.63321-3.38122-0.60383$

$\mathrm{N}-2.35690-1.806400 .96997$

N -2.42980 -3.07904 0.66294

C -2.36901-4.11332 1.67986

Br -3.11865 -3.13214 -3.88255

Mg - 2.362521 .740931 .01575

C -6.606911 .235412 .03294$

C -5.405970 .980331 .13931$

O -4.302761 .653381 .79421$

C -4.795342 .544402 .82692$

C -6.295722 .618812 .60220$

H -6.650780 .493772 .83418$

H - - .544121 .196841 .47740

H -5.53559 1.418070 .14732

H $-5.14303-0.070101 .03323$

H -4.283643 .498932 .71825$

H -4.542742 .108283 .79454$

H -6.534053 .394491 .87058$

$\mathrm{H}-6.835422 .838783 .52348$

H -3.02951 -0.14496 -3.85683

H $-2.711351 .54580-2.14259$

H $-3.37458-4.342852 .03156$

H - $1.76354-3.747262 .50362$

H - $1.91924-4.999461 .24137$

Br - 1.204151 .762483 .27460

Benzotriazole dimer delocalized radical anion coordination (10)

Electronic energy calculated $(E)=-12031.29246$

Standard state Gibbs free energy $\left(G^{\circ}\right)=-12030.89600$

Number of imaginary frequencies (Nimag) $=0$ 
C -2.33690 -0.49795 2.01927

C $-0.94591-0.770601 .85310$

C $-0.13088-0.045051 .01393$

C -0.766321 .014170 .29872$

C - 2.152331 .291640 .45177

C -2.908550 .518711 .33776$

Br -5.25755 3.83109 2.38578

N -2.46670 2.31357-0.36307

$\mathrm{N}-0.279811 .87980-0.60821$

$\mathrm{N}-1.328402 .59726-0.94787$

C $-1.212883 .70596-1.87920$

C $1.30973-0.332670 .87373$

C $1.85074-1.642121 .03073$

C 2.233730 .646320 .58840

C 3.626220 .398200 .46570

C $4.13735-0.855050 .62181$

C $3.23669-1.900630 .90465$

N $1.24782-2.817871 .28596$

N $3.44350-3.213201 .08683$

N 2.23617 -3.67942 1.30734

Br $5.99205-1.210620 .46140$

C $1.99425-5.098411 .50860$

H -2.91203 -1.10981 2.70463

H $-0.52450-1.588812 .42119$

H $-0.440013 .46545-2.60321$

H - $0.947454 .61250-1.33646$

H -2.17144 $3.83562-2.37236$

H 1.897311 .663450 .44617

H 4.283701 .230240 .24701

H $2.93669-5.562361 .78103$

H $1.61226-5.534500 .58672$

H $1.26508-5.216932 .30531$

C -5.92842 -1.29994-1.56635

C $-5.779870 .05634-1.56095$

C $-5.639170 .77698-0.33697$

C -5.639940 .198810 .91943$

C $-5.80471-1.212100 .86782$

C -5.94662 -1.96660 - 0.32513

$\mathrm{N}-6.08234-3.26316-0.00972$

$\mathrm{N}-5.86387-2.091831 .89164$

$\mathrm{N}-6.01826-3.255471 .30726$

C $-6.19022-4.475692 .07462$

Br -6.11239-2.29177 -3.18306

Mg -5.62349 1.35113 2.73836

C -9.964201 .109263 .20519$

C -8.700450 .950512 .37850$ 


$$
\begin{array}{lll}
\text { O }-7.62410 & 1.35375 & 3.26286 \\
\mathrm{C}-8.15366 & 2.02224 & 4.43669 \\
\mathrm{C}-9.61278 & 2.28900 & 4.11085 \\
\mathrm{H}-10.14579 & 0.21133 & 3.80053 \\
\mathrm{H}-10.83901 & 1.29018 & 2.58051 \\
\mathrm{H}-8.69436 & 1.61147 & 1.50948 \\
\mathrm{H}-8.50319 & -0.06918 & 2.05409 \\
\mathrm{H}-7.56455 & 2.92244 & 4.60405 \\
\mathrm{H}-8.03507 & 1.34945 & 5.28731 \\
\mathrm{H}-9.71616 & 3.23109 & 3.56737 \\
\mathrm{H}-10.22756 & 2.34137 & 5.00947 \\
\mathrm{H}-5.75979 & 0.59965 & -2.49901 \\
\mathrm{H}-5.49937 & 1.85207 & -0.42730 \\
\mathrm{H}-7.24696 & -4.62723 & 2.29397 \\
\text { H }-5.63062 & -4.37959 & 3.00040 \\
\text { H }-5.81143 & -5.30598 & 1.48579 \\
\text { Br }-4.65590 & 0.63763 & 4.95919
\end{array}
$$

Benzotriazole dimer radical anion C-C bond formation transition state (TS-4)

Electronic energy calculated $(E)=-12031.29335$

Standard state Gibbs free energy $\left(G^{\circ}\right)=-12030.89483$

Number of imaginary frequencies $(\mathrm{Nimag})=1$

C $-2.54374-0.564342 .07497$

C $-1.15160-0.810151 .90868$

C $-0.33973-0.028851 .11792$

C -0.995101 .047640 .45122$

C -2.387471 .285100 .61136$

C -3.169250 .481801 .46605$

$\mathrm{Br}-5.050673 .763432 .45718$

$\mathrm{N}-2.712212 .34161-0.15546$

$\mathrm{N}-0.522871 .94457-0.43346$

$\mathrm{N}-1.583492 .66336-0.73645$

C $-1.490743 .81125-1.61966$

C $1.10518-0.282160 .97345$

C $1.66301-1.592471 .02659$

C 2.019090 .730330 .79095

C 3.418270 .512960 .68113

C $3.94583-0.742280 .73777$

C $3.05417-1.820990 .90081$

N $1.07142-2.793881 .16255$

N $3.27493-3.142770 .95886$

N 2.07038-3.64220 1.11471

Br $5.81107-1.057870 .60183$

C $1.84684-5.077341 .16401$

H -3.10344 -1.22513 2.72727 


$$
\begin{array}{llll}
\text { H }-0.72096 & -1.65082 & 2.43826 \\
\mathrm{H}-0.71092 & 3.61902 & -2.35118 \\
\mathrm{H}-1.24769 & 4.70101 & -1.04006 \\
\mathrm{H}-2.44972 & 3.94232 & -2.11086 \\
\mathrm{H}-1.66875 & 1.75079 & 0.73012 \\
\mathrm{H}-4.06714 & 1.37024 & 0.55490 \\
\mathrm{H}-2.74511 & -5.54599 & 1.55480 \\
\mathrm{H}-1.63778 & -5.44675 & 0.16146 \\
\mathrm{H}-0.99943 & -5.26963 & 1.81567 \\
\mathrm{C}-6.04607 & -1.57541 & -1.47242 \\
\mathrm{C}-5.85177 & -0.22676 & -1.56274 \\
\mathrm{C}-5.51305 & 0.54701 & -0.41705 \\
\mathrm{C}-5.38276 & 0.03036 & 0.85710 \\
\mathrm{C}-5.60204 & -1.37057 & 0.92018 \\
\mathrm{C}-5.92328 & -2.17570 & -0.20281 \\
\mathrm{~N}-6.06995 & -3.44594 & 0.19946 \\
\mathrm{~N}-5.57613 & -2.18881 & 1.99102 \\
\mathrm{~N}-5.84702 & -3.37291 & 1.49582 \\
\mathrm{C}-5.97892 & -4.54178 & 2.34730 \\
\mathrm{Br}-6.47521 & -2.63239 & -2.99394 \\
\mathrm{Mg}-5.26623 & 1.23843 & 2.68320 \\
\mathrm{C}-9.63567 & 1.44413 & 2.75111 \\
\mathrm{C}-8.31641 & 1.22441 & 2.03468 \\
\mathrm{O}-7.31572 & 1.26169 & 3.08227 \\
\mathrm{C}-7.86812 & 1.86522 & 4.28240 \\
\mathrm{C}-9.23138 & 2.39826 & 3.87383 \\
\mathrm{H}-10.00800 & 0.50303 & 3.16145 \\
\mathrm{H}-10.39660 & 1.85484 & 2.08719 \\
\mathrm{H}-8.10301 & 2.02480 & 1.32490 \\
\mathrm{H}-8.23291 & 0.26592 & 1.52824 \\
\mathrm{H}-7.17944 & 2.63622 & 4.62315 \\
\mathrm{H}-7.93645 & 1.08671 & 5.04217 \\
\mathrm{H}-9.14284 & 3.41612 & 3.48938 \\
\mathrm{H}-9.93128 & 2.40454 & 4.70940 \\
\mathrm{H}-5.94558 & 0.26826 & -2.52280 \\
\mathrm{H}-5.33719 & 1.60313 & -0.59066 \\
\mathrm{H}-7.01814 & -4.65945 & 2.65036 \\
\mathrm{H}-5.35118 & -4.39740 & 3.22065 \\
\mathrm{~B}-5.65586 & -5.41323 & 1.78536 \\
& -4.52656 & 0.53535 & 5.01353
\end{array}
$$

\section{Benzotriazole trimer radical anion product (11)}

Electronic energy calculated $(E)=-12031.38067$

Standard state Gibbs free energy $\left(G^{\circ}\right)=-12030.97788$

Number of imaginary frequencies $(\mathrm{Nimag})=0$ 
C - $2.45671-1.213461 .27991$

C $-1.06456-1.194971 .34680$

C $-0.25229-0.258350 .70848$

C $-0.990930 .70400-0.05345$

C $-2.411660 .68606-0.13780$

C -3.22151 -0.28569 0.55140

$\mathrm{Br}-4.226963 .494012 .10590$

N -2.78068 $1.67690-0.96298$

$\mathrm{N}-0.552071 .71156-0.82521$

$\mathrm{N}-1.650732 .23310-1.33062$

C - $1.616843 .41649-2.16656$

C $1.20272-0.257680 .82225$

C $1.95691-1.362261 .34486$

C 1.984330 .820930 .43545

C 3.394820 .859400 .54585

C $4.10034-0.192941 .04983$

C $3.37076-1.328611 .45298$

N $1.56063-2.568271 .79276$

N $3.79081-2.495791 .96006$

N 2.67556 -3.16680 2.13706

Br $5.99082-0.161101 .20626$

C $2.68380-4.536702 .61930$

H -2.97034 -1.98236 1.83414

H - $0.59587-1.962841 .94435$

H - $0.723453 .37818-2.78399$

$\mathrm{H}-1.598734 .31176-1.54468$

H -2.50606 $3.41825-2.79016$

H 1.500791 .692040 .02127

H 3.913011 .753850 .22210

H $3.53498-4.658313 .28257$

H $2.76772-5.221031 .77563$

H $1.75568-4.716743 .15334$

C $-7.53883-0.55475-0.04774$

C $-6.813960 .54908-0.44495$

C $-5.444900 .67094-0.19295$

C $-4.66109-0.329490 .47832$

C $-5.44977-1.474810 .90663$

C $-6.84787-1.575130 .64541$

N -7.29093 -2.73462 1.13441

$\mathrm{N}-5.08778-2.593581 .54110$

$\mathrm{N}-6.20223-3.295821 .64091$

C $-6.25197-4.548862 .36344$

Br $-9.39479-0.72595-0.40744$

Mg -5.04448 1.17501 2.36197

C -9.259162 .113552 .69744$

C -7.830232 .484022 .34644$ 
$\begin{array}{lll}\text { O }-7.03329 & 1.33255 & 2.75076 \\ \text { C }-7.83724 & 0.41940 & 3.55328 \\ \text { C }-9.07494 & 1.21547 & 3.91978 \\ \text { H }-9.72189 & 1.55628 & 1.88038 \\ \text { H }-9.86525 & 2.99654 & 2.89962 \\ \text { H }-7.47522 & 3.34674 & 2.91035 \\ \text { H }-7.66007 & 2.65114 & 1.28509 \\ \text { H }-7.23304 & 0.10721 & 4.40127 \\ \text { H }-8.07564 & -0.44680 & 2.93446 \\ \text { H }-8.89197 & 1.81576 & 4.81385 \\ \text { H }-9.93012 & 0.56679 & 4.10903 \\ \text { H }-7.30912 & 1.35190 & -0.97868 \\ \text { H }-4.93765 & 1.53568 & -0.59023 \\ \text { H }-6.40393 & -4.36668 & 3.42826 \\ \text { H }-5.31215 & -5.07323 & 2.21206 \\ \text { H }-7.07663 & -5.13643 & 1.96952 \\ \text { Br -4.23029 } & 0.04925 & 4.41674\end{array}$

Benzotriazole tetramer radical anion

Electronic energy calculated $(E)=-6884.061022$

Standard state Gibbs free energy $\left(G^{\circ}\right)=-6883.659259$

Number of imaginary frequencies (Nimag) $=0$

$\begin{array}{lrrr}\mathrm{C} & -2.56863 & -1.24127 & -0.42934 \\ \mathrm{C} & -1.21775 & -1.03480 & -0.18786 \\ \mathrm{C} & -0.64122 & 0.21686 & 0.11871 \\ \mathrm{C} & -1.62250 & 1.27562 & 0.15164 \\ \mathrm{C} & -3.01108 & 1.05642 & -0.09896 \\ \mathrm{C} & -3.55051 & -0.23951 & -0.40392 \\ \mathrm{C} & -4.95485 & -0.49606 & -0.66236 \\ \mathrm{~N} & -3.63429 & 2.23933 & 0.01763 \\ \mathrm{~N} & -1.46232 & 2.58187 & 0.40991 \\ \mathrm{~N} & -2.67322 & 3.08763 & 0.32457 \\ \mathrm{C} & -2.92172 & 4.50811 & 0.45393 \\ \mathrm{C} & 0.75571 & 0.40404 & 0.36343 \\ \mathrm{C} & 1.73563 & -0.65603 & 0.33741 \\ \mathrm{C} & 1.33450 & 1.65779 & 0.65723 \\ \mathrm{C} & 2.68595 & 1.86441 & 0.89535 \\ \mathrm{C} & 3.66643 & 0.86099 & 0.87816 \\ \mathrm{C} & 3.12502 & -0.43649 & 0.58332 \\ \mathrm{~N} & 1.57096 & -1.96666 & 0.10557 \\ \mathrm{~N} & 3.74423 & -1.62357 & 0.49011 \\ \mathrm{~N} & 2.77772 & -2.47725 & 0.21687 \\ \mathrm{C} & 5.07186 & 1.11830 & 1.12999 \\ \mathrm{C} & 3.04663 & -3.87463 & -0.05015 \\ \mathrm{H} & -2.87371 & -2.25237 & -0.65264\end{array}$




$\begin{array}{cccc}\mathrm{H} & -0.57096 & -1.89605 & -0.24087 \\ \mathrm{H} & -2.16269 & 4.92981 & 1.10679 \\ \mathrm{H} & -3.90850 & 4.64890 & 0.88713 \\ \mathrm{H} & -2.87675 & 4.98985 & -0.52369 \\ \mathrm{H} & 0.68934 & 2.52076 & 0.70094 \\ \mathrm{H} & 2.99288 & 2.87725 & 1.10803 \\ \mathrm{H} & 3.87329 & -4.19432 & 0.57883 \\ \mathrm{H} & 3.30707 & -4.01494 & -1.10004 \\ \mathrm{H} & 2.15349 & -4.44608 & 0.18654 \\ \mathrm{C} & -5.92000 & 0.51042 & -0.66815 \\ \mathrm{C} & -7.29325 & 0.29599 & -0.91558 \\ \mathrm{C} & -7.79131 & -0.94873 & -1.17444 \\ \mathrm{C} & -6.87557 & -2.01922 & -1.18688 \\ \mathrm{C} & -5.49367 & -1.80400 & -0.93828 \\ \mathrm{~N} & -4.89638 & -3.00792 & -1.01208 \\ \mathrm{~N} & -7.07636 & -3.32599 & -1.40175 \\ \mathrm{~N} & -5.87353 & -3.84212 & -1.27842 \\ \mathrm{C} & -5.62779 & -5.25452 & -1.50507 \\ \mathrm{Br} & -9.63533 & -1.25489 & -1.50741 \\ \mathrm{C} & 5.60673 & 2.42140 & 1.43524 \\ \mathrm{C} & 6.99011 & 2.63759 & 1.67506 \\ \mathrm{C} & 7.91150 & 1.57322 & 1.62328 \\ \mathrm{C} & 7.41721 & 0.33301 & 1.33708 \\ \mathrm{C} & 6.04244 & 0.11743 & 1.09917 \\ \mathrm{~N} & 5.00342 & 3.61884 & 1.55207 \\ \mathrm{~N} & 5.97850 & 4.45076 & 1.83207 \\ \mathrm{~N} & 7.18585 & 3.93862 & 1.92622 \\ \mathrm{C} & 5.72741 & 5.85425 & 2.10363 \\ \mathrm{Br} & 9.75733 & 1.88088 & 1.94470 \\ \mathrm{H} & -5.61025 & 1.52428 & -0.47147 \\ \mathrm{H} & -7.95885 & 1.15083 & -0.89684 \\ \mathrm{H} & -4.75062 & -5.54371 & -0.93412 \\ \mathrm{H} & -5.45711 & -5.43298 & -2.56649 \\ \mathrm{H} & -6.49887 & -5.81101 & -1.17122 \\ \mathrm{H} & 8.08713 & -0.51724 & 1.28776 \\ \mathrm{H} & 5.73615 & -0.89280 & 0.87977 \\ & 4.83965 & 6.15405 & 1.55500 \\ \mathrm{H} & 5.58965 & 6.42616 & 1.77285 \\ \mathrm{H} & & & 3.17233\end{array}$

Benzotriazole tetramer radical anion chain-end meta carbon monomer coordination

Electronic energy calculated $(E)=-12899.02746$

Standard state Gibbs free energy $\left(G^{\circ}\right)=-12898.41063$

Number of imaginary frequencies $(\mathrm{Nimag})=0$ 


$$
\begin{aligned}
& \text { C }-1.77533-1.47724-0.59620 \\
& \text { C }-0.45948-1.21278-0.18685 \\
& \text { C } 0.036840 .062550 .09403 \\
& \text { C }-0.953801 .09397-0.08160 \\
& \text { C - }-2.282840 .81800-0.50604 \\
& \text { C }-2.74505-0.50626-0.77940 \\
& \text { C -4.10633 -0.81259-1.22979 } \\
& \mathrm{N}-2.949711 .98341-0.55272 \\
& \mathrm{~N}-0.864872 .421100 .11363 \\
& \mathrm{~N}-2.060992 .87388-0.17331 \\
& \text { C - } 2.364664 .29242-0.15204 \\
& \text { C } 1.397020 .305280 .51421 \\
& \text { C } 2.37720-0.729570 .71204 \\
& \text { C } 1.906861 .592400 .77488 \\
& \text { C } 3.204121 .864121 .17406 \\
& \text { C } 4.198740 .879391 .37859 \\
& \text { C } 3.71356-0.453011 .12753 \\
& \text { N } 2.27093-2.058230 .55756 \\
& \text { N } 4.36320-1.621451 .20891 \\
& \text { N } 3.46860-2.522350 .85474 \\
& \text { C } 5.531441 .175111 .79145 \\
& \text { C } 3.75179-3.941560 .90329 \\
& \text { H -2.03264 -2.51097-0.77815 } \\
& \text { H } 0.20366-2.05719-0.08705 \\
& \mathrm{H}-1.758934 .760850 .61823 \\
& \text { H -3.42057 4.411820.07250 } \\
& \text { H -2.13885 } 4.73458-1.12229 \\
& \text { H } 1.244382 .436170 .65895 \\
& \text { H } 3.464492 .898281 .33733 \\
& \text { H } 4.82152-4.079200 .77130 \\
& \text { H } 3.20831-4.433680 .10080 \\
& \text { H } 3.44333-4.355111 .86420 \\
& \text { C }-4.931050 .13094-1.81248 \\
& \text { C -6.24471 -0.14203 -2.26980 } \\
& \text { C }-6.79313-1.38440-2.15628 \\
& \text { C }-6.00652-2.39158-1.56350 \\
& \text { C }-4.69260-2.11339-1.11190 \\
& \mathrm{~N}-4.21082-3.25177-0.57864 \\
& \mathrm{~N}-6.28640-3.67618-1.30133 \\
& \mathrm{~N}-5.19134-4.11090-0.72237 \\
& \text { C }-5.04692-5.50000-0.32171 \\
& \text { Br }-8.54876-1.77062-2.75911 \\
& \text { C } 6.013802 .511212 .06996 \\
& \text { C } 7.309472 .762152 .59742 \\
& \text { C } 8.212911 .707352 .86822 \\
& \text { C } 7.799730 .442212 .56887
\end{aligned}
$$


C 6.523160 .175162 .00993

N 5.418093 .702871 .91866

N 6.315934 .574372 .32436

N 7.46326 4.07810 2.74971

C 6.028755 .993482 .38830

Br 9.92960 2.07226 3.58527

H -4.56879 1.14097 -1.93079

H -6.81251 $0.66263-2.72042$

H - $4.36110-5.541970 .51894$

H $-4.65303-6.08077-1.15499$

H -6.02506 -5.87519-0.03605

H $8.45810-0.397482 .76005$

H $6.22440-0.862371 .94629$

H 5.359846 .247121 .57066

H 6.965736 .534122 .29035

H 5.558286 .236433 .34141

C $9.44225-4.73780-0.34357$

C $8.88650-4.242330 .79778$

C $8.31803-2.931250 .83246$

C $8.27394-2.06193-0.23856$

C $8.86344-2.62061-1.40511$

C $9.43774-3.91534-1.48696$

N $9.88771-4.11645-2.73459$

N $8.98292-2.06653-2.63307$

N $9.57981-2.99018-3.34593$

C $9.96006-2.75888-4.72812$

Br $10.20518-6.48109-0.41988$

Mg $7.42008-0.09492-0.24961$

C $4.394820 .50559-3.29711$

C $5.145501 .04493-2.09283$

O $5.84213-0.10860-1.53963$

C $5.35424-1.33586-2.15089$

C $4.08456-0.93049-2.87624$

H $5.034590 .51444-4.18257$

H $3.501831 .09234-3.51210$

H $4.472641 .42389-1.32306$

H $5.892771 .79875-2.33057$

H $5.20562-2.06323-1.35625$

H $6.12350-1.69679-2.83487$

H $3.23014-0.95559-2.19645$

H $3.87274-1.58912-3.71848$

H $8.87203-4.847121 .69733$

H $7.89531-2.636681 .79023$

H $10.94311-2.29043-4.76456$

H $9.22218-2.10481-5.18331$

H $9.98632-3.71698-5.23857$ 
Br $8.658561 .95257-0.94575$

Benzotriazole tetramer inner-ring meta carbon monomer coordination radical anion

Electronic energy calculated $(E)=-12899.03912$

Standard state Gibbs free energy $\left(\mathrm{G}^{\circ}\right)=-12898.40627$

Number of imaginary frequencies $($ Nimag) $=0$

C $-1.50649-1.69511-0.22648$

C $-0.20123-1.349780 .06066$

C $0.35841-0.07435-0.19500$

C $-0.532800 .79406-0.89747$

C $-1.893570 .45289-1.16017$

C $-2.45189-0.82418-0.81413$

C -3.83033 -1.19090 -1.04663

$\mathrm{N}-2.459471 .49602-1.78393$

$\mathrm{N}-0.316132 .01405-1.43428$

$\mathrm{N}-1.508202 .38604-1.92101$

C - $1.760533 .67666-2.53064$

C 1.639870 .337110 .30948

C $2.67497-0.579270 .70009$

C 1.993751 .675150 .51511

C 3.221742 .090761 .04487

C 4.239871 .227041 .42715

C $3.92013-0.153551 .23518$

N 2.71311 -1.91800 0.59497

N $4.67102-1.248991 .44123$

N $3.90380-2.241751 .03999$

C 5.510851 .688711 .98571

C $4.32283-3.624281 .16714$

$\mathrm{H}-1.82617-2.688490 .04687$

H $0.41558-2.088670 .55073$

H $-0.973464 .36523-2.23348$

H -2.71767 $4.04243-2.16651$

H - $1.779013 .58651-3.61553$

H 1.263092 .446490 .31372

H 3.369433 .155691 .15911

H $5.39427-3.677060 .99400$

H $3.79055-4.210840 .42428$

H $4.09089-3.992192 .16676$

C -4.77751 -0.30684-1.57328

C $-6.13012-0.63364-1.80037$

C -6.63004 - $1.87403-1.51724$

C -5.73416 -2.82403-0.98739

C $-4.37089-2.49645-0.75574$

$\mathrm{N}-3.78926-3.59998-0.25230$

$\mathrm{N}-5.93959-4.09599-0.62285$ 
$\mathrm{N}-4.75704-4.48518-0.19705$

C $-4.51688-5.850220 .23201$

$\mathrm{Br}-8.44955-2.32809-1.81661$

C 6.006003 .019541 .80193

C 7.231753 .455162 .36418

C 8.019032 .579543 .13760

C 7.560151 .307413 .31216

C 6.335350 .877222 .74470

N 5.502644 .054091 .10364

N 6.387275 .007971 .26525

N 7.438034 .729942 .00411

C 6.178036 .335900 .71414

Br 9.65557 3.17285 3.89153

H -4.46903 0.69407 -1.82709

$\mathrm{H}-6.781410 .12948-2.20987$

H -3.69599 -5.84541 0.94320

H -4.26044 -6.46824 -0.62824

H -5.42222 -6.22587 0.70024

H 8.133000 .601023 .90044

H $6.04186-0.146052 .92423$

H $5.641536 .23856-0.22453$

H 7.149306 .793750 .55379

H 5.593976 .933221 .41348

C $2.630117 .35283-0.70228$

C $1.647876 .59696-0.13644$

C $1.321395 .30598-0.65487$

C $1.936614 .71106-1.73790$

C $2.946745 .53741-2.30008$

C $3.307226 .82191-1.81739$

N $4.305607 .30864-2.57028$

N $3.750875 .28225-3.35696$

N $4.510696 .34530-3.44808$

C $5.479206 .49920-4.51900$

Br 3.09437 9.07299-0.02915

Mg $1.465432 .75695-2.47869$

C $4.52498-0.14813-3.37007$

C $3.076620 .13644-3.01575$

O $3.073341 .50937-2.53942$

C $4.435101 .97202-2.30504$

C $5.285600 .71695-2.36550$

H $4.736680 .17711-4.39121$

H $4.76065-1.20932-3.29118$

H $2.71753-0.49863-2.20422$

H $2.391410 .07614-3.85892$

H $4.452572 .47861-1.34256$

H $4.684422 .68226-3.09399$ 
H $5.320360 .23205-1.38744$

H $6.307300 .93622-2.67558$

H 1.103436 .974360 .72169

H $0.526744 .78206-0.12747$

H $4.987016 .90393-5.40277$

H $5.901635 .52460-4.74543$

H $6.253977 .18102-4.18136$

Br $0.735052 .45723-4.88557$

\section{Benzotriazole tetramer excited state geometry: ortho monomer coordinated radical anion}

Electronic energy calculated $(E)=-12899.01635$

Standard state Gibbs free energy $\left(G^{\circ}\right)=-12898.3169$

Number of imaginary frequencies (Nimag) $=0$

C $-0.93952-2.20163-0.23658$

C $0.31085-1.804370 .24885$

C $0.85714-0.538940 .06916$

C $0.013400 .34490-0.67221$

C $-1.26271-0.05666-1.17648$

C $-1.79539-1.37455-0.96709$

C -3.08794 -1.81134-1.46249

$\mathrm{N}-1.789570 .99106-1.82506$

N $0.213191 .62176-1.03309$

$\mathrm{N}-0.884051 .94007-1.69466$

C - $1.029783 .21889-2.34276$

C $2.16872-0.149420 .59688$

C $3.13404-1.082821 .09712$

C 2.602521 .161030 .64974

C 3.873121 .557051 .13174

C 4.829690 .683051 .60273

C $4.41112-0.683491 .58003$

N $3.06992-2.423121 .18373$

N $5.07175-1.792371 .95260$

N $4.22929-2.764381 .69217$

C 6.164741 .121922 .04921

C $4.58876-4.156161 .87693$

H - $1.25192-3.21240-0.02735$

H $0.88209-2.539360 .79612$

H $-0.537373 .97361-1.73368$

H -2.09093 $3.43733-2.43254$

H $-0.575483 .20201-3.33643$

H 1.945411 .938230 .29096

H 4.090652 .614541 .13139

H $5.49045-4.186512 .48088$

H $4.77585-4.613370 .90605$ 
H $3.77072-4.667772 .37980$

C $-3.93821-0.98146-2.19803$

C -5.19424 - $1.37765-2.68930$

C $-5.69087-2.63741-2.47959$

C $-4.88975-3.53507-1.74807$

C $-3.61510-3.13360-1.24808$

$\mathrm{N}-3.10275-4.18931-0.59771$

$\mathrm{N}-5.11405-4.80457-1.39436$

$\mathrm{N}-4.02774-5.12132-0.71450$

C -3.81467-6.45773 -0.21407

$\mathrm{Br}-7.38747-3.17221-3.14143$

C 6.487412 .492062 .30341

C 7.780772 .891192 .76062

C 8.798241 .944012 .96140

C 8.507860 .620502 .66884

C 7.217380 .233682 .24031

N 5.744063 .602242 .18877

N 6.571494 .580372 .55166

N 7.796224 .220642 .91873

C 6.105465 .932512 .71457

Br 10.46342 2.46927 3.67599

H -3.61993 $0.02725-2.40721$

H -5.78162 -0.65973 -3.24993

H $-3.24123-6.395630 .70771$

H -3.26531 -7.05585 - 0.94408

H $-4.78657-6.90820-0.03045$

H $9.24457-0.148352 .87692$

H $7.04367-0.820252 .08305$

H 5.354436 .136971 .95430

H 6.955076 .601932 .60213

H 5.662106 .072003 .70457

C $7.91671-4.50004-0.54896$

C $9.14054-4.10045-0.10530$

C $9.47366-2.71538-0.00458$

C $8.62497-1.67856-0.33394$

C $7.37028-2.13600-0.81836$

C $6.99657-3.50142-0.92426$

N $5.74011-3.57766-1.38502$

N $6.31250-1.41063-1.23964$

N $5.40726-2.31154-1.54168$

C $4.11714-1.94498-2.09410$

Br 7.43935 -6.33947 -0.66258

Mg 9.22113 0.31687 0.08118

C $7.898194 .04294-1.67027$

C $8.788123 .27822-0.70419$

O $8.239251 .92939-0.68074$ 
C $6.966491 .88261-1.38537$

C $6.552163 .33552-1.51254$

H $8.266703 .94274-2.69464$

H $7.858965 .10551-1.42761$

H 8.732323 .676550 .30962

H $9.831943 .20592-1.00066$

H $6.287241 .25823-0.81295$

H $7.138181 .41323-2.35638$

H $6.047283 .66278-0.60126$

H $5.877643 .49417-2.35479$

H $9.87462-4.843920 .18429$

H $10.47342-2.496020 .36333$

H $4.13388-2.06663-3.17765$

H $3.91872-0.91001-1.83381$

H $3.35554-2.58673-1.66023$

Br 11.574960 .944790 .04059

\section{Benzotriazole hexamer radical anion}

Electronic energy calculated $(E)=-7751.806528$

Standard state Gibbs free energy $\left(G^{\circ}\right)=-7751.187484$

Number of imaginary frequencies (Nimag) $=0$

C -2.311410 .233780 .19939$

C $-1.75411-0.994620 .39361$

C $-0.34847-1.170330 .43848$

C $0.56327-0.140400 .29841$

C -0.032241 .145060 .08736$

C -1.437021 .326330 .03697$

$\mathrm{N}-1.693922 .62295-0.18622$

$\mathrm{N}-0.497353 .15887-0.25867$

N $0.526892 .35320-0.11354$

C $-0.313744 .56893-0.55683$

C $2.01215-0.349780 .36725$

C $2.63702-1.622160 .18611$

C 2.910010 .669860 .63665

C 4.298570 .496700 .73378

C $4.95428-0.724900 .56378$

C $4.04548-1.804350 .27246$

N $2.08657-2.81166-0.10987$

N $4.29562-3.101960 .02496$

N $3.11443-3.62631-0.19359$

C $2.94065-5.04579-0.43705$

C $6.38744-0.875320 .67319$

C 7.294700 .205000 .95420

C 8.706510 .021381 .06021

C $9.34628-1.261790 .89597$ 
C $8.41877-2.288530 .62063$ C $7.04784-2.105540 .51773$

N 7.040121 .505461 .16600

N 9.248271 .218051 .33167

N 8.223412 .039661 .38864

C 8.395763 .464741 .57993

C $10.75772-1.479230 .99826$

C $11.39860-2.762590 .83763$

C $11.68387-0.452351 .27102$

C $13.05651-0.636101 .37575$

C $13.71508-1.864711 .22603$

C $12.80980-2.945710 .94700$

N $10.85764-3.960360 .56814$

N $11.88223-4.781350 .51622$

N $13.06500-4.247140 .73937$

C $15.15183-2.015041 .34096$

C $16.05903-0.931501 .61725$

C $17.46601-1.114801 .71059$

C $18.08790-2.390191 .55332$

C $17.19388-3.413191 .29711$

C $15.80370-3.238361 .19180$

N 15.811360 .371221 .84228

N 16.993560 .895342 .05480

N 18.019680 .077841 .98798

C 17.169772 .318542 .27604

C $19.53821-2.597531 .63250$

C $20.12645-3.867061 .93026$

C $20.45056-1.583261 .41782$

C $21.85608-1.761801 .47317$

C $22.40763-2.975981 .75388$

C $21.52978-4.051871 .99151$

N $19.55941-5.052332 .22388$

N $21.77925-5.329492 .31244$

N $20.57971-5.848592 .43569$

C $20.39202-7.259722 .72668$

C $11.71241-6.207320 .32645$

Br 24.28406 -3.23551 1.83520

Br -4.18889 0.491220.13634

H - $2.38765-1.863610 .52196$

H $0.01724-2.174710 .58959$

$\mathrm{H}-1.215285 .09544-0.25957$

H - $0.140564 .69570-1.62481$

H 0.542784 .932180 .00377

H 2.530391 .670420 .78712

H 4.888941 .370580 .95888

H 2.12605 -5.17840 -1.14388 
H 2.70701 -5.55601 0.49734

H $3.86646-5.43464-0.85011$

H $8.79267-3.290210 .47918$

H $6.45407-2.980530 .30232$

H 7.500903 .858152 .05422

H 8.551483 .957730 .61950

H 9.25984 3.62464 2.21911

H 11.309950 .549621 .40951

H 13.649770 .239901 .58895

H $17.57357-4.416561 .16407$

H $15.21224-4.114390 .97806$

H 16.247512 .714112 .69058

H 17.396202 .814081 .33212

H 17.990222 .460312 .97411

H $20.08744-0.590661 .19678$

H $22.49383-0.907491 .28260$

H $19.45160-7.375783 .25672$

H $21.22173-7.593323 .34260$

H $20.36813-7.823861 .79504$

H $10.81492-6.36942-0.26375$

H $11.61483-6.705871 .29154$

H $12.58206-6.59191-0.19958$

Benzotriazole hexamer radical anion meta carbon chain-end (ring 1) monomer coordination

Electronic energy calculated $(E)=-13766.77177$

Standard state Gibbs free energy $\left(\mathrm{G}^{\circ}\right)=-13765.90886$

Number of imaginary frequencies (Nimag) $=0$

$\begin{array}{rrrr}\mathrm{C} & -2.24422 & 1.23852 & -0.62820 \\ \mathrm{C} & -1.73405 & -0.01643 & -0.78877 \\ \mathrm{C} & -0.38003 & -0.32619 & -0.49929 \\ \mathrm{C} & 0.58532 & 0.64516 & -0.10341 \\ \mathrm{C} & 0.00638 & 1.95524 & 0.10680 \\ \mathrm{C} & -1.36176 & 2.23818 & -0.15472 \\ \mathrm{~N} & -1.59696 & 3.51959 & 0.13003 \\ \mathrm{~N} & -0.42489 & 3.96374 & 0.54589 \\ \mathrm{~N} & 0.56143 & 3.09321 & 0.54772 \\ \mathrm{C} & -0.21731 & 5.35562 & 0.89175 \\ \mathrm{C} & 1.97528 & 0.35745 & 0.03393 \\ \mathrm{C} & 2.55149 & -0.94285 & -0.19188 \\ \mathrm{C} & 2.94301 & 1.31996 & 0.40517 \\ \mathrm{C} & 4.29454 & 1.05455 & 0.54504 \\ \mathrm{C} & 4.89337 & -0.20439 & 0.34422 \\ \mathrm{C} & 3.94358 & -1.21488 & -0.03969 \\ \mathrm{~N} & 1.95131 & -2.08583 & -0.54860\end{array}$




\begin{tabular}{|c|c|c|c|}
\hline $\mathrm{N}$ & 4.13099 & -2.51581 & -0.31002 \\
\hline $\mathrm{N}$ & 2.92652 & -2.97154 & -0.59239 \\
\hline $\mathrm{C}$ & 2.70545 & -4.33106 & -1.03916 \\
\hline $\mathrm{C}$ & 6.30914 & -0.44251 & 0.50588 \\
\hline $\mathrm{C}$ & 7.26742 & 0.57553 & 0.85398 \\
\hline $\mathrm{C}$ & 8.65701 & 0.30615 & 0.98947 \\
\hline $\mathrm{C}$ & 9.21044 & -0.99870 & 0.81046 \\
\hline $\mathrm{C}$ & 8.26708 & -1.95879 & 0.48731 \\
\hline $\mathrm{C}$ & 6.89612 & -1.69871 & 0.33621 \\
\hline $\mathrm{N}$ & 7.08724 & 1.88209 & 1.11539 \\
\hline $\mathrm{N}$ & 9.26806 & 1.45372 & 1.32801 \\
\hline $\mathrm{N}$ & 8.28951 & 2.32845 & 1.38797 \\
\hline $\mathrm{C}$ & 8.53812 & 3.73190 & 1.65895 \\
\hline $\mathrm{C}$ & 10.64049 & -1.29234 & 0.93255 \\
\hline $\mathrm{C}$ & 11.15012 & -2.59655 & 1.22099 \\
\hline $\mathrm{C}$ & 11.62105 & -0.33320 & 0.76994 \\
\hline $\mathrm{C}$ & 13.00495 & -0.60312 & 0.86105 \\
\hline $\mathrm{C}$ & 13.52291 & -1.84837 & 1.14037 \\
\hline $\mathrm{C}$ & 12.53862 & -2.86153 & 1.33064 \\
\hline $\mathrm{N}$ & 10.49715 & -3.75119 & 1.44701 \\
\hline $\mathrm{N}$ & 11.45567 & -4.61920 & 1.66092 \\
\hline $\mathrm{N}$ & 12.68730 & -4.16806 & 1.61244 \\
\hline $\mathrm{C}$ & 14.96734 & -2.10365 & 1.25459 \\
\hline $\mathrm{C}$ & 15.94492 & -1.34484 & 0.54649 \\
\hline $\mathrm{C}$ & 17.33296 & -1.59754 & 0.67171 \\
\hline $\mathrm{C}$ & 17.84175 & -2.61553 & 1.52842 \\
\hline $\mathrm{C}$ & 16.87704 & -3.32986 & 2.20090 \\
\hline $\mathrm{C}$ & 15.48950 & -3.08846 & 2.06305 \\
\hline $\mathrm{N}$ & 15.79082 & -0.33019 & -0.32352 \\
\hline $\mathrm{N}$ & 17.01827 & -0.02061 & -0.66353 \\
\hline $\mathrm{N}$ & 17.98244 & -0.72587 & -0.12060 \\
\hline $\mathrm{C}$ & 17.29647 & 1.00642 & -1.65280 \\
\hline $\mathrm{C}$ & 19.28121 & -2.89647 & 1.66871 \\
\hline $\mathrm{C}$ & 19.84309 & -3.44352 & 2.85978 \\
\hline $\mathrm{C}$ & 20.18350 & -2.66163 & 0.65585 \\
\hline $\mathrm{C}$ & 21.57095 & -2.94165 & 0.76204 \\
\hline $\mathrm{C}$ & 22.10145 & -3.46540 & 1.90264 \\
\hline $\mathrm{C}$ & 21.22607 & -3.71994 & 2.97675 \\
\hline $\mathrm{N}$ & 19.27182 & -3.76424 & 4.03528 \\
\hline $\mathrm{N}$ & 21.46179 & -4.20043 & 4.20648 \\
\hline $\mathrm{N}$ & 20.27432 & -4.19301 & 4.76475 \\
\hline $\mathrm{C}$ & 20.06659 & -4.69736 & 6.11203 \\
\hline $\mathrm{C}$ & 11.16819 & -5.99923 & 2.01049 \\
\hline $\mathrm{Br}$ & 23.95002 & -3.84438 & 2.06780 \\
\hline $\mathrm{Br}$ & -4.06108 & 1.64912 & -0.98212 \\
\hline $\mathrm{H}$ & -2.37316 & -0.81407 & -1.14989 \\
\hline
\end{tabular}




$\begin{array}{cccc}\mathrm{H} & -0.02728 & -1.30381 & -0.79864 \\ \mathrm{H} & -1.13632 & 5.74149 & 1.32399 \\ \mathrm{H} & 0.04091 & 5.92753 & 0.00001 \\ \mathrm{H} & 0.59169 & 5.41199 & 1.61433 \\ \mathrm{H} & 2.61585 & 2.33043 & 0.59462 \\ \mathrm{H} & 4.92780 & 1.88057 & 0.82976 \\ \mathrm{H} & 1.66942 & -4.59013 & -0.83870 \\ \mathrm{H} & 3.37088 & -4.99224 & -0.48994 \\ \mathrm{H} & 2.90488 & -4.41358 & -2.10826 \\ \mathrm{H} & 8.59365 & -2.97765 & 0.33378 \\ \mathrm{H} & 6.26458 & -2.52990 & 0.06577 \\ \mathrm{H} & 7.66345 & 4.14649 & 2.15169 \\ \mathrm{H} & 8.72421 & 4.26215 & 0.72505 \\ \mathrm{H} & 9.40747 & 3.80554 & 2.30588 \\ \mathrm{H} & 11.32609 & 0.68489 & 0.56262 \\ \mathrm{H} & 13.68328 & 0.22301 & 0.69954 \\ \mathrm{H} & 17.17984 & -4.11935 & 2.87448 \\ \mathrm{H} & 14.81545 & -3.71793 & 2.62628 \\ \mathrm{H} & 16.49361 & 1.73651 & -1.61965 \\ \mathrm{H} & 17.34939 & 0.55457 & -2.64268 \\ \mathrm{H} & 18.24595 & 1.47172 & -1.40496 \\ \mathrm{H} & 19.83292 & -2.24147 & -0.27578 \\ \mathrm{H} & 22.20827 & -2.73334 & -0.08804 \\ \mathrm{H} & 19.27670 & -4.11521 & 6.57731 \\ \mathrm{H} & 20.99712 & -4.58759 & 6.66007 \\ \mathrm{H} & 19.77975 & -5.74716 & 6.06665 \\ \mathrm{H} & 10.21865 & -6.27349 & 1.56107 \\ \mathrm{H} & 11.11026 & -6.09805 & 3.09397 \\ \mathrm{H} & 11.96772 & -6.62382 & 1.62291 \\ \mathrm{C} & -2.67630 & -5.96717 & 0.52764 \\ \mathrm{C} & -2.32350 & -5.09436 & -0.45759 \\ \mathrm{C} & -1.83197 & -3.78900 & -0.14516 \\ \mathrm{C} & -1.67014 & -3.29152 & 1.13201 \\ \mathrm{C} & -2.04950 & -4.23504 & 2.12515 \\ \mathrm{C} & -2.54047 & -5.54014 & 1.86291 \\ \mathrm{~N} & -2.78776 & -6.15708 & 3.02811 \\ \mathrm{~N} & -2.01071 & -4.09987 & 3.47028 \\ \mathrm{~N} & -2.44763 & -5.24993 & 3.92179 \\ & -2.62617 & -5.49219 & 5.34231 \\ \mathrm{Cr} & -3.33283 & -7.71273 & 0.14145 \\ \mathrm{C} & 2.450977 & -1.34876 & 1.65369 \\ \mathrm{H} & -1.39828 & 4.36990 \\ \mathrm{H} & -0.59875 & 3.47337 \\ \mathrm{H} & -1.59719 & 2.68334 \\ \mathrm{H} & -2.90156 & 2.83684 \\ \mathrm{H} & -2.61662 & 3.50574\end{array}$




$\begin{array}{cccc}\mathrm{H} & 1.93333 & -1.70329 & 5.28229 \\ \mathrm{H} & 3.33414 & -0.82469 & 4.65081 \\ \mathrm{H} & 2.06970 & 0.04255 & 2.78145 \\ \mathrm{H} & 0.77823 & -0.01222 & 4.00682 \\ \mathrm{H} & 1.52209 & -3.34745 & 1.84813 \\ \mathrm{H} & 0.78949 & -3.51399 & 3.45988 \\ \mathrm{H} & 3.53272 & -2.36812 & 2.75998 \\ \mathrm{H} & 3.12766 & -3.47132 & 4.08265 \\ \mathrm{H} & -2.41505 & -5.38829 & -1.49701 \\ \mathrm{H} & -1.57296 & -3.17549 & -1.00495 \\ \mathrm{H} & -3.60052 & -5.12047 & 5.65803 \\ \mathrm{H} & -1.84008 & -4.97298 & 5.88298 \\ \mathrm{H} & -2.56354 & -6.56251 & 5.51414 \\ \mathrm{Br} & -2.16386 & 0.26800 & 3.09141\end{array}$

Benzotriazole hexamer radical anion inner ring (ring 2) meta carbon monomer coordination

Electronic energy calculated $(E)=-13766.77407$

Standard state Gibbs free energy $\left(\mathrm{G}^{\circ}\right)=-13765.94129$

Number of imaginary frequencies (Nimag) $=0$

C $-2.549950 .77557-0.70296$

C $-1.99532-0.44198-0.96788$

C - $0.62237-0.69832-0.74186$

C $0.274050 .23869-0.24322$

C -0.322411 .518010 .02905$

C -1.69934 1.77667-0.19349

$\mathrm{N}-1.962763 .040760 .16515$

$\mathrm{N}-0.797763 .486700 .57790$

N 0.210772 .650870 .52153

C -0.618874 .861541 .00928$

C $1.68215-0.05259-0.02150$

C $2.27103-1.35591-0.16849$

C 2.604180 .917950 .36803

C 3.968150 .683900 .59302

C $4.60414-0.576450 .39767$

C $3.66002-1.608190 .03906$

N $1.69154-2.52182-0.48038$

N $3.86612-2.91788-0.15384$

N $2.67688-3.39860-0.44685$

C $2.47805-4.78514-0.81406$

C $6.01180-0.773650 .51637$

C 6.941010 .269950 .86856

C 8.341670 .052091 .01210

C $8.95001-1.231600 .79382$

C $8.02108-2.216880 .43302$ 
C $6.65220-2.012140 .30526$

N 6.702281 .561031 .13769

N 8.896751 .222181 .36855

N 7.882292 .060071 .43158

C 8.06619 3.47016 1.71029

C $10.37231-1.491980 .93154$

C $10.98693-2.769340 .68631$

C $11.28694-0.517411 .31916$

C $12.66887-0.739641 .46248$

C $13.28341-1.957011 .24387$

C $12.38214-2.989320 .84351$

N $10.44404-3.929120 .27435$

N $11.46032-4.752840 .19608$

N $12.63661-4.269120 .52188$

C $14.72427-2.169381 .42535$

C $15.68441-1.114251 .34783$

C $17.07217-1.337941 .52954$

C $17.60095-2.628741 .82014$

C $16.65445-3.625431 .89327$

C $15.27158-3.406591 .69629$

N 15.513520 .196661 .09748

N 16.727610 .687621 .14433

N $17.70117-0.157441 .39048$

C 16.991412 .088030 .86204

C $19.03988-2.882162 .00464$

C 19.53466 -3.95149 2.80912

C $20.01129-2.112231 .40435$

C $21.40292-2.349051 .55212$

C $21.86845-3.373662 .32014$

C $20.92070-4.191642 .96614$

N $18.88562-4.866953 .55194$

N 21.08134 -5.23870 3.78848

N $19.84941-5.575134 .09078$

C $19.56198-6.722144 .93601$

C $11.28373-6.14936-0.15766$

Br 23.72115 -3.70831 2.53335

Br -4.39048 1.13506 -0.99461

H - $2.61050-1.24260-1.36044$

H $-0.26510-1.68959-0.97104$

H - 1.530355 .187631 .50183

H -0.415795 .493690 .14543$

H 0.218934 .893071 .69967

H 2.259041 .933300 .50013

H 4.592701 .548900 .77134

H $1.49034-5.09237-0.48114$

H $3.24142-5.38123-0.32250$ 
H $2.55605-4.90252-1.89562$

H $8.38340-3.215160 .24126$

H $6.04873-2.861980 .02843$

H 7.140933 .855962 .12907

H 8.307674 .005580 .79160

H 8.878063 .579572 .42438

H 10.925100 .476541 .52822

H 13.273260 .103161 .76633

H $16.96990-4.635662 .11390$

H $14.61905-4.264861 .76135$

H 16.144062 .672481 .20823

H $17.126022 .22566-0.21028$

H 17.894642 .376691 .39096

H $19.71471-1.278170 .78484$

H $22.09702-1.697261 .03679$

H $18.68616-6.495045 .53690$

H $20.42582-6.897645 .56944$

H $19.37085-7.594104 .31201$

H $10.42710-6.22771-0.82070$

H $11.11280-6.739460 .74234$

H $12.18431-6.49146-0.65943$

C 3.732625 .587954 .37304

C 3.647055 .224413 .06224

C 3.603103 .848002 .67948

C 3.640022 .783783 .55743

C 3.731503 .210014 .90996

C 3.779504 .562455 .33686

N 3.863834 .596486 .67545

N 3.792832 .450226 .02754

N 3.862283 .320637 .00511

C 4.004582 .903358 .38863

Br 3.79365 7.41402 4.91259

Mg 3.554610 .710923 .01731

C $6.70579-2.002484 .24528$

C $5.26636-1.747703 .84585$

O $5.15466-0.298703 .78525$

C 6.272820 .324374 .47962

C $6.99660-0.820175 .16928$

H $7.35300-1.977113 .36591$

H $6.82531-2.968994 .73522$

H $4.55797-2.108644 .59279$

H $4.99792-2.142922 .86880$

H 5.868851 .070225 .16103

H 6.895250 .812093 .72947

H $6.57338-0.998366 .16030$

H $8.06076-0.613835 .28315$ 
H 3.611375 .986982 .29252

H 3.534413 .667791 .60942

H 5.060552 .785928 .63061

H 3.486941 .957348 .51670

H 3.562503 .666889 .02185

Br $1.67867-0.762253 .74794$

Benzotriazole hexamer radical anion inner ring (ring 3) meta carbon monomer coordination

Electronic energy calculated $(E)=-13766.77415239$

Standard state Gibbs free energy $\left(G^{\circ}\right)=-13765.93651$

Number of imaginary frequencies (Nimag) $=0$

C $-1.940131 .91848-0.60003$

C $-1.461920 .66193-0.82724$

C $-0.095560 .34419-0.64304$

C $0.868421 .25299-0.22751$

C 0.352232 .573410 .01040

C $-1.017882 .89362-0.17187$

$\mathrm{N}-1.202994 .184040 .13793$

$\mathrm{N}-0.002704 .586250 .48740$

$\mathrm{N} 0.960253 .697350 .43407$

C 0.260535 .969180 .84427

C $2.270740 .89174-0.05495$

C $2.76427-0.45357-0.11387$

C 3.277891 .832470 .17089

C 4.627081 .528840 .34057

C 5.154800 .224000 .30600

C $4.14247-0.770580 .05462$

N $2.10390-1.60794-0.30434$

N $4.26551-2.10198-0.04493$

N $3.04073-2.53203-0.24546$

C $2.75313-3.93240-0.48464$

C $6.53737-0.078470 .49886$

C 7.564830 .907290 .73264

C 8.951480 .580740 .82276

C $9.44879-0.764780 .71217$

C $8.42876-1.705820 .53485$

C $7.06575-1.400890 .45111$

N 7.448942 .230120 .90957

N 9.620031 .718131 .04911

N 8.683972 .646491 .09795

C 9.006364 .050261 .24360

C $10.85035-1.123810 .77746$

C $11.32675-2.477870 .84560$

C $11.87902-0.186260 .77619$

C $13.24553-0.515740 .82878$ 
C $13.73051-1.807900 .90507$

C $12.70717-2.803910 .91857$

N $10.63974-3.633070 .86609$

N $11.56467-4.557730 .94594$

N $12.81044-4.142880 .97920$

C $15.16140-2.120520 .98906$

C $16.18375-1.239480 .51905$

C $17.56177-1.557990 .61447$

C $18.02092-2.771491 .20327$

C $17.01630-3.600631 .64827$

C $15.64116-3.292071 .53858$

N $16.08548-0.03548-0.07375$

N $17.330160 .31155-0.29182$

N $18.25822-0.537400 .08349$

C $17.664941 .54328-0.98490$

C $19.44713-3.121851 .30903$

C $19.95334-3.976782 .33291$

C $20.39325-2.658390 .42200$

C $21.76949-2.997630 .49502$

C $22.24565-3.816661 .47439$

C $21.32492-4.316122 .41643$

N $19.33198-4.574553 .36626$

N 21.50411 -5.10661 3.48484

N $20.29677-5.208263 .98926$

C $20.02772-6.040625 .15000$

C $11.23378-5.970140 .99440$

Br 24.07905 -4.28044 1.59298

Br $-3.768132 .36468-0.83923$

H -2.13273 -0.12123-1.15910

H $0.20083-0.67396-0.84077$

H -0.640046 .382561 .28836$

H $0.528736 .53306-0.04860$

H 1.080175 .988681 .55633

H 3.004132 .875550 .22495

H 5.302322 .352510 .51024

H $1.78515-4.16787-0.05029$

H $3.53286-4.52247-0.01146$

H $2.73572-4.13237-1.55626$

H $8.70274-2.746270 .43523$

H $6.39550-2.210040 .19292$

H 8.167974 .546871 .72419

H 9.19029 4.49874 0.26637

H 9.89652 4.136071 .86081

H 11.624950 .861450 .73855

H 13.949450 .304180 .81091

H 17.27772 -4.54198 2.11121 
H $14.93672-4.023201 .90691$

H $16.895132 .27731-0.76680$

H $17.710631 .35885-2.05774$

H $18.630671 .88559-0.62502$

H $20.08761-1.99919-0.37752$

H $22.44298-2.59320-0.25008$

H $19.21618-5.589385 .71296$

H $20.93129-6.085885 .74998$

H $19.74483-7.040194 .82248$

H $10.15169-6.055090 .99239$

H $11.64302-6.404551 .90434$

H $11.65425-6.471530 .12446$

C $6.26216-7.241072 .36923$

C $6.68075-6.495191 .30790$

C $6.83857-5.079161 .41780$

C $6.59555-4.344302 .56059$

C $6.15817-5.161623 .63771$

C $5.98604-6.568863 .57521$

N $5.56569-7.012184 .76973$

N $5.82581-4.787934 .89477$

N $5.50013-5.910035 .48886$

C $5.01927-5.936366 .85869$

Br 6.04604 -9.13001 2.25268

Mg 6.85537 -2.22555 2.76602

C $2.93422-1.127964 .27571$

C $3.84308-2.076083 .50991$

O $5.10420-1.362083 .36666$

C 4.968950 .003853 .84625

C 3.473710 .247073 .88290

H $3.03860-1.284315 .35173$

H $1.88699-1.270864 .00943$

H $3.47137-2.287612 .50756$

H $4.04515-3.010864 .02851$

H 5.510010 .651493 .15980

H 5.423520 .060694 .83630

H 3.113360 .538612 .89412

H 3.207511 .030634 .59243

H $6.90037-6.978260 .36250$

H 7.17886 -4.58093 0.51348

H $3.93725-5.807466 .87146$

H $5.49569-5.127737 .40506$

H $5.28255-6.896057 .29349$

Br 8.44460 -1.20783 4.39749

Benzotriazole hexamer radical anion excited state geometry ortho monomer coordination

Electronic energy calculated $(E)=-13766.76103$ 
Standard state Gibbs free energy $\left(G^{\circ}\right)=-13765.82638$

Number of imaginary frequencies $($ Nimag $)=0$

C -3.09017 $0.80861-1.55506$

C $-2.63584-0.45370-1.24704$

C $-1.26682-0.71007-0.99487$

C $-0.277780 .26716-1.01720$

C $-0.762321 .58615-1.29583$

C $-2.137251 .84472-1.56785$

$\mathrm{N}-2.293423 .15429-1.79850$

$\mathrm{N}-1.072063 .63112-1.65685$

$\mathrm{N}-0.118672 .76296-1.36657$

C $-0.768715 .01678-1.93000$

C $1.13774-0.03617-0.77348$

C $1.65982-1.36635-0.69699$

C $2.088970 .94564-0.56951$

C $3.448660 .68081-0.28904$

C $3.97859-0.58667-0.19450$

C $3.02850-1.62594-0.41966$

N $1.04272-2.55122-0.83668$

N $3.19577-2.95956-0.38879$

N $1.99834-3.43060-0.64178$

C $1.74044-4.85743-0.65756$

C $5.39612-0.842800 .11133$

C 6.221140 .088880 .80715

C $7.59500-0.165051 .08217$

C $8.25294-1.375010 .67382$

C $7.41298-2.252450 .00728$

C $6.05052-1.99617-0.25864$

N 5.905011 .282491 .33613

N 8.066410 .886811 .77201

N 7.030711 .683121 .88687

C 7.144112 .980372 .51376

C $9.66035-1.660740 .93302$

C $10.27866-2.923520 .66198$

C $10.54207-0.724991 .46287$

C $11.89586-0.961821 .71459$

C $12.54192-2.176511 .47124$

C $11.66006-3.174130 .92465$

N $9.74976-4.047000 .15432$

N $10.76298-4.888260 .11995$

N $11.91491-4.441060 .56757$

C $13.94329-2.397401 .74414$

C $14.82534-1.396152 .28012$

C $16.21017-1.643302 .53424$

C $16.82857-2.912422 .27782$

C $15.93867-3.856051 .76057$ 
C $14.58892-3.616571 .50925$

N $14.56948-0.128962 .63386$

N 15.725050 .323843 .07268

N $16.73962-0.516513 .03175$

C 15.897841 .699603 .46801

C $18.23155-3.194702 .52478$

C $18.84224-4.477822 .29045$

C $19.12003-2.239003 .01202$

C $20.48671-2.481333 .26096$

C $21.05840-3.701733 .03822$

C $20.22313-4.721852 .54237$

N $18.32140-5.630091 .83768$

N $20.50026-5.995242 .24035$

N $19.33662-6.465511 .84119$

C $19.20053-7.815081 .34360$

C $10.58999-6.26150-0.28819$

Br $22.89602-4.032193 .37469$

Br -4.88791 1.15515 -2.00221

H -3.32732 -1.28907 -1.26174

H $-0.98254-1.73430-0.80649$

H - $1.662275 .60352-1.73439$

H - $0.470755 .13984-2.97308$

H $0.046915 .32933-1.28229$

H $1.792001 .98120-0.63126$

H $4.097751 .52881-0.12466$

H $0.70811-5.00115-0.96034$

H $1.89501-5.261030 .34156$

H $2.41804-5.33501-1.36316$

H $7.81493-3.19494-0.32972$

H $5.49628-2.76256-0.78192$

H 6.183363 .231702 .95569

H 7.41652 3.735071 .77420

H 7.91450 2.92311 3.27799

H 10.166170 .257611 .70378

H $12.47172-0.147232 .12382$

H $16.31044-4.843481 .53444$

H $14.01110-4.432881 .10576$

H 14.954612 .060543 .87035

H 16.187352 .312482 .61144

H 16.676161 .745054 .22604

H $18.74825-1.247493 .21592$

H $21.09451-1.668303 .64068$

H $18.20593-8.176041 .59277$

H $19.96173-8.427981 .81865$

H $19.33559-7.834340 .26086$

H $9.84821-6.29555-1.08263$ 
H $10.25043-6.869750 .55256$

H $11.54713-6.63297-0.64463$

C $-0.98779-5.252292 .27536$

C -2.29664 -4.98640 2.01030

C - $2.76470-3.643991 .87477$

C - $-1.97095-2.522221 .99502$

C $-0.61874-2.838242 .29282$

C $-0.11349-4.157442 .42641$

N $1.20193-4.098912 .67626$

N $0.42332-2.001332 .48175$

N $1.44198-2.803422 .68975$

C $2.76900-2.298132 .98671$

$\mathrm{Br}-0.33667-7.031762 .43777$

Mg -2.82025 -0.62497 1.61834

C -1.548513 .326292 .79900$

C - 2.516702 .421692 .05594

O -1.864461 .119282 .03403$

C -0.484131 .230042 .48910$

C -0.190662 .717152 .44908$

H - -1.726013 .275953 .87636$

H - 1.648974 .367022 .48902

H -2.66448 2.73616 1.02194

H -3.48743 2.29878 2.53020

H 0.134970 .620281 .83808

H - 0.432760 .823343 .50081

H 0.119513 .012901 .44473

H 0.600562 .993663 .14667

H - 2.99722 -5.80557 1.89507

H -3.82539 -3.53391 1.66118

H $2.82076-1.266382 .65318$

H $3.49928-2.897392 .45005$

H $2.95318-2.359244 .05957$

Br -5.18032 -0.14338 1.88149

\section{Benzotriazole Grignard monomer one THF coordination}

Electronic energy calculated $(E)=-6014.965061$

Standard state Gibbs free energy $\left(G^{\circ}\right)=-6014.77198$

Number of imaginary frequencies (Nimag) $=0$

C 6.723742 .618543 .17235

C 6.899621 .268243 .22646

C 6.081650 .385122 .45547

C 5.070500 .793291 .61007

C 4.917462 .205721 .58750

C 5.706513 .116242 .33411

N 5.294104 .365812 .07303 
N 4.031332 .945020 .88324

$\mathrm{N} 4.314244 .182591 .21242$

C 3.537445 .301950 .70906

Br 7.79712 3.81618 4.18803

Mg 3.82002 -0.46109 0.44910

C $3.001540 .41607-3.71423$

C $2.79856-0.45723-2.48959$

O $3.683740 .10828-1.47872$

C $4.588641 .07978-2.08283$

C $4.461740 .84490-3.57545$

H $2.342951 .28656-3.67679$

H $2.79905-0.12853-4.63609$

H $3.10613-1.48908-2.66197$

H $1.78543-0.44315-2.09313$

H $5.585290 .89954-1.68425$

H $4.254192 .07328-1.78432$

H $5.129740 .04170-3.89450$

H $4.702761 .74043-4.14771$

H 7.67010 0.850123 .86379

H $6.29995-0.673542 .57006$

H 2.64478 5.432971 .31976

H $3.255185 .09397-0.31914$

H 4.156436 .192320 .76055

Br 2.67413 -2.61787 0.64904

Benzotriazole Grignard monomer two THF coordination

Electronic energy calculated $(E)=-6247.376935$

Standard state Gibbs free energy $\left(\mathrm{G}^{\circ}\right)=-6247.067759$

Number of imaginary frequencies (Nimag) $=0$

C 6.994622 .200823 .21290

C 6.987870 .840143 .14203

C 6.005540 .146542 .36862

C 5.001720 .755301 .64203

C 5.044292 .172431 .74809

C 5.999852 .900892 .50191

N 5.749594 .212542 .37425

N 4.226403 .084651 .17469

N 4.699634 .238881 .57884

C 4.053525 .492151 .23215

Br 8.29098 3.15178 4.23170

Mg $3.54855-0.288060 .47656$

C $2.906290 .64729-3.69502$

C $2.77640-0.30778-2.52437$

O $3.575880 .29445-1.47148$

C $4.469971 .30391-2.02433$ 


$$
\begin{array}{llll}
\text { C } 4.33553 & 1.16431 & -3.53132 \\
\text { H } 2.18794 & 1.46542 & -3.60364 \\
\text { H } 2.73742 & 0.14698 & -4.64823 \\
\text { H } 3.19167 & -1.29193 & -2.74667 \\
\text { H } 1.76156 & -0.42317 & -2.14637 \\
\text { H } 5.47241 & 1.10724 & -1.64867 \\
\text { H } 4.13344 & 2.27478 & -1.65997 \\
\text { H } 5.05051 & 0.43225 & -3.91269 \\
\text { H } 4.51028 & 2.11119 & -4.04154 \\
\text { H } 7.73707 & 0.26846 & 3.67763 \\
\text { H } 6.08605 & -0.93785 & 2.37503 \\
\text { H } 3.25939 & 5.70646 & 1.94688 \\
\text { H } 3.63795 & 5.40167 & 0.23271 \\
\text { H } 4.80114 & 6.27900 & 1.26275 \\
\text { Br } 3.40051 & -2.77530 & 0.51523 \\
\text { C }-0.31584 & 1.65754 & 0.94804 \\
\text { C } 1.07391 & 1.57090 & 0.33607 \\
\text { O } 1.62275 & 0.30658 & 0.81082 \\
\text { C } 0.71818 & -0.30806 & 1.76707 \\
\text { C }-0.17721 & 0.82637 & 2.22327 \\
\text { H }-1.05675 & 1.20952 & 0.28242 \\
\text { H }-0.60849 & 2.68978 & 1.13888 \\
\text { H } 1.73941 & 2.36308 & 0.67832 \\
\text { H } 1.07035 & 1.54778 & -0.75284 \\
\text { H } 1.31876 & -0.75262 & 2.55826 \\
\text { H } 0.16067 & -1.09286 & 1.25275 \\
\text { H } 0.31358 & 1.40449 & 3.00951 \\
\text { H }-1.13150 & 0.46434 & 2.60539
\end{array}
$$

Benzotriazole dimer radical anion lithium and two THF coordinated

Electronic energy calculated $(E)=-6488.586991$

Standard state Gibbs free energy $\left(\mathrm{G}^{\circ}\right)=-6488.178622$

Number of imaginary frequencies (Nimag) $=0$

$$
\begin{array}{llll}
\text { C } & -1.81959 & -0.75706 & 0.87569 \\
\text { C }-0.48594 & -0.58322 & 0.54713 \\
\text { C } & 0.06586 & 0.63916 & 0.08173 \\
\text { C }-0.87738 & 1.71505 & -0.03518 \\
\text { C }-2.25924 & 1.52909 & 0.30677 \\
\text { C }-2.72237 & 0.30062 & 0.76302 \\
\text { Br }-4.57510 & 0.11997 & 1.18887 \\
\text { N }-2.91129 & 2.68332 & 0.11408 \\
\text { N }-0.73102 & 2.98419 & -0.42437 \\
\text { N }-1.93819 & 3.55054 & -0.26684 \\
\text { C }-2.27255 & 4.76668 & -0.96636 \\
\text { C } 1.46901 & 0.78489 & -0.25265
\end{array}
$$




$$
\begin{aligned}
& \text { C } 2.43937-0.27405-0.13177 \\
& \text { C } 2.012251 .98048-0.72693 \\
& \text { C } 3.371742 .15833-1.06283 \\
& \text { C } 4.280841 .14612-0.94525 \\
& \text { C } 3.80741-0.09307-0.46962 \\
& \text { N } 2.30910-1.549050 .27905 \\
& \text { N } 4.45931-1.24357-0.25839 \\
& \text { N } 3.51675-2.052190 .17465 \\
& \text { Br } 6.11227 \text { 1.36928 -1.39393 } \\
& \text { C } 3.81252-3.410110 .59276 \\
& \text { H -2.16003 -1.72449 } 1.22525 \\
& \text { H } 0.16800-1.433470 .65839 \\
& \text { H - } 1.407275 .42435-0.94334 \\
& \text { H }-3.105535 .25135-0.45960 \\
& \text { H - } 2.547904 .56279-2.00601 \\
& \text { H } 1.362302 .83180-0.84914 \\
& \text { H } 3.689683 .12982-1.42261 \\
& \text { H } 4.68035-3.753960 .03792 \\
& \text { H } 2.95055-4.033600 .37317 \\
& \text { H } 4.02250-3.429531 .66195 \\
& \text { H -4.90624 2.11803-2.31802 } \\
& \text { H -3.34362 4.23284 2.58189 } \\
& \text { H -4.28687 3.50998 4.69167 } \\
& \text { H -6.09004 3.81236 -3.57658 } \\
& \text { C }-4.336954 .602952 .83221 \\
& \text { C }-4.718374 .430414 .29130 \\
& \mathrm{H}-7.085332 .40276-3.96869 \\
& \text { C }-5.968602 .23559-2.10696 \\
& \text { C }-6.727293 .03186-3.15384 \\
& \text { H }-6.431492 .433573 .13367 \\
& \text { H - } 4.380475 .264574 .90616 \\
& \text { O -5.30204 3.817342.09554 } \\
& \text { H -6.41246 1.24977 -1.94766 } \\
& \text { C }-6.438393 .508082 .93541 \\
& \text { O -6.09855 2.99883 -0.88549 } \\
& \text { H }-4.423225 .645232 .51347 \\
& \text { C -6.24069 4.31923 4.20782 } \\
& \text { Li -4.83731 } 2.896820 .51138 \\
& \text { H -6.68594 3.83237 5.07529 } \\
& \text { C -7.85469 3.64876 -2.32624 } \\
& \mathrm{H}-6.694224 .97203-1.04370 \\
& \text { C - } 7.152063 .98030-1.01831 \\
& \text { H - - . } .347763 .763742 .39135 \\
& \text { H -8.29622 } 4.53162-2.78832 \\
& \text { H }-6.689005 .310014 .10378 \\
& \text { H -8.64791 2.91643 -2.15913 }
\end{aligned}
$$


H -7.79944 $3.90955-0.14440$

Lithium cation three THF coordination

Electronic energy calculated $(E)=-704.6560818$

Standard state Gibbs free energy $\left(G^{\circ}\right)=-704.3300805$

Number of imaginary frequencies (Nimag) $=0$

$\begin{array}{llll}\mathrm{C} & -3.20086 & -1.18832 & -2.62359 \\ \mathrm{C} & -3.72732 & -2.15805 & -1.57977 \\ \mathrm{O} & -3.04965 & -1.80050 & -0.35344 \\ \mathrm{C} & -1.93104 & -0.92683 & -0.63732 \\ \mathrm{C} & -1.76641 & -0.95927 & -2.14817 \\ \mathrm{H} & -3.76681 & -0.25421 & -2.59711 \\ \mathrm{H} & -3.26111 & -1.59787 & -3.63173 \\ \mathrm{H} & -3.47343 & -3.19216 & -1.82689 \\ \mathrm{H} & -4.80080 & -2.08522 & -1.40757 \\ \mathrm{H} & -1.06220 & -1.29403 & -0.09143 \\ \mathrm{H} & -2.18020 & 0.07515 & -0.27991 \\ \mathrm{H} & -1.12757 & -1.79371 & -2.44662 \\ \mathrm{H} & -1.32697 & -0.03847 & -2.53120 \\ \mathrm{C} & -1.21031 & -0.72417 & 4.45073 \\ \mathrm{C} & -1.78972 & -1.93527 & 3.73787 \\ \mathrm{O} & -2.74525 & -1.40279 & 2.78997 \\ \mathrm{C} & -2.97395 & 0.00173 & 3.04698 \\ \mathrm{C} & -2.38160 & 0.25736 & 4.42224 \\ \mathrm{H} & -0.36090 & -0.32142 & 3.89411 \\ \mathrm{H} & -0.87439 & -0.96545 & 5.45904 \\ \mathrm{H} & -2.32278 & -2.59342 & 4.42814 \\ \mathrm{H} & -1.05092 & -2.51848 & 3.18858 \\ \mathrm{H} & -4.04570 & 0.18785 & 2.98394 \\ \mathrm{H} & -2.46340 & 0.58274 & 2.27453 \\ \mathrm{H} & -3.10614 & 0.01139 & 5.20205 \\ \mathrm{H} & -2.07742 & 1.29586 & 4.55149 \\ \mathrm{Li} & -3.47638 & -2.41250 & 1.37651 \\ \mathrm{C} & -2.71245 & -5.18224 & 0.72625 \\ \mathrm{O} & -3.41447 & -4.28011 & 1.61392 \\ \mathrm{C} & -4.00901 & -5.01726 & 2.70673 \\ \mathrm{C} & -3.95357 & -6.47428 & 2.28120 \\ \mathrm{H} & -5.01875 & -4.63822 & 2.86179 \\ \mathrm{H} & -3.41665 & -4.83847 & 3.60786 \\ \mathrm{C} & -2.66705 & -6.51463 & 1.45694 \\ \mathrm{H} & -4.81576 & -6.72322 & 1.65808 \\ \mathrm{H} & -3.94031 & -7.15116 & 3.13524 \\ \mathrm{H} & -2.62271 & -7.35748 & 0.76752 \\ \mathrm{H} & -1.79498 & -6.56225 & 2.11323 \\ & -1.72923 & -4.76122 & 0.51710\end{array}$




\section{$\mathrm{H} \quad-3.27684 \quad-5.25240 \quad-0.20666$ \\ Thiophene dimer radical anion $\mathrm{Li}(\mathrm{THF})_{2}$ coordinated}

Electronic energy calculated $(E)=-6488.58699$

Standard state Gibbs free energy $\left(\mathrm{G}^{\circ}\right)=-6488.16252$

Number of imaginary frequencies (Nimag) $=0$

C -1.81959 -0.75706 0.87569

C $-0.48594-0.583220 .54713$

C 0.065860 .639160 .08173

C $-0.877381 .71505-0.03518$

C - 2.259241 .529090 .30677

C -2.722370 .300620 .76302$

$\mathrm{Br}-4.575100 .119971 .18887$

N -2.91129 2.683320.11408

$\mathrm{N}-0.731022 .98419-0.42437$

$\mathrm{N}-1.938193 .55054-0.26684$

C - $2.272554 .76668-0.96636$

C $1.469010 .78489-0.25265$

C $2.43937-0.27405-0.13177$

C $2.012251 .98048-0.72693$

C $3.371742 .15833-1.06283$

C $4.280841 .14612-0.94525$

C $3.80741-0.09307-0.46962$

N $2.30910-1.549050 .27905$

N $4.45931-1.24357-0.25839$

N $3.51675-2.052190 .17465$

Br 6.11227 1.36928 -1.39393

C $3.81252-3.410110 .59276$

H - $2.16003-1.724491 .22525$

H $0.16800-1.433470 .65839$

H - $1.407275 .42435-0.94334$

H -3.10553 $5.25135-0.45960$

H -2.54790 4.56279 -2.00601

H $1.362302 .83180-0.84914$

H $3.689683 .12982-1.42261$

H 4.68035 -3.75396 0.03792

H $2.95055-4.033600 .37317$

H $4.02250-3.429531 .66195$

$\mathrm{H}-4.906242 .11803-2.31802$

H -3.34362 4.23284 2.58189

$\mathrm{H}-4.286873 .509984 .69167$

$\mathrm{H}-6.090043 .81236-3.57658$

C -4.336954 .602952 .83221$

C -4.718374 .430414 .29130$

H -7.08533 2.40276 -3.96869 
$\begin{array}{llll}\text { C }-5.96860 & 2.23559 & -2.10696 \\ \text { C }-6.72729 & 3.03186 & -3.15384 \\ \text { H }-6.43149 & 2.43357 & 3.13367 \\ \text { H }-4.38047 & 5.26457 & 4.90616 \\ \text { O }-5.30204 & 3.81734 & 2.09554 \\ \text { H }-6.41246 & 1.24977 & -1.94766 \\ \text { C }-6.43839 & 3.50808 & 2.93541 \\ \text { O }-6.09855 & 2.99883 & -0.88549 \\ \text { H }-4.42322 & 5.64523 & 2.51347 \\ \text { C }-6.24069 & 4.31923 & 4.20782 \\ \text { Li }-4.83731 & 2.89682 & 0.51138 \\ \text { H }-6.68594 & 3.83237 & 5.07529 \\ \text { C }-7.85469 & 3.64876 & -2.32624 \\ \text { H }-6.69422 & 4.97203 & -1.04370 \\ \text { C -7.15206 } & 3.98030 & -1.01831 \\ \text { H }-7.34776 & 3.76374 & 2.39135 \\ \text { H }-8.29622 & 4.53162 & -2.78832 \\ \text { H }-6.68900 & 5.31001 & 4.10378 \\ \text { H }-8.64791 & 2.91643 & -2.15913 \\ \text { H }-7.79944 & 3.90955 & -0.14440\end{array}$

Thiophene dimer radical anion meta coordination conformer 1

Electronic energy calculated $(E)=-12502.88080307$

Standard state Gibbs free energy $\left(G^{\circ}\right)=-12502.60141$

Number of imaginary frequencies $($ Nimag $)=0$

C $-4.203671 .26491-0.78392$

C $-3.702860 .04417-0.51533$

C $-2.339560 .10362-0.00031$

C $-1.779971 .42927-0.14678$

S $-3.071242 .59240-0.49205$

C $-0.446671 .83036-0.12687$

C $0.106663 .11058-0.23975$

C $1.517663 .15102-0.21569$

C $2.051221 .89697-0.08751$

S 0.849420 .636890 .00659

Br 3.881511 .462450 .03063

$\mathrm{Br}-5.955761 .69909-1.34743$

C $2.320904 .41397-0.30919$

H 2.918044 .573950 .59181

H $1.668145 .27757-0.43716$

H $3.013474 .38598-1.15363$

H $-0.500554 .00266-0.33356$

C $-4.40947-1.25832-0.73039$

H $-5.48863-1.13240-0.81449$ 


$$
\begin{array}{lll}
\text { H }-4.20196 & -1.95188 & 0.08606 \\
\mathrm{H}-4.05483 & -1.72985 & -1.65155 \\
\mathrm{H}-1.69217 & -0.74485 & -0.23087 \\
\mathrm{C}-2.79627 & -4.80299 & 2.57918 \\
\mathrm{C}-2.21342 & -3.52940 & 2.26471 \\
\mathrm{C}-2.94576 & -2.40963 & 2.57089 \\
\mathrm{~S}-4.43746 & -2.95181 & 3.28477 \\
\mathrm{C}-4.02053 & -4.62780 & 3.14491 \\
\mathrm{H}-1.23137 & -3.47109 & 1.80191 \\
\mathrm{Mg}-2.54686 & -0.31518 & 2.25309 \\
\mathrm{Br}-5.18175 & -5.99908 & 3.73592 \\
\mathrm{Br}-0.74344 & 0.65683 & 3.71430 \\
\mathrm{C}-2.13789 & -6.12443 & 2.31707 \\
\mathrm{C}-4.33240 & 0.67269 & 4.54684 \\
\mathrm{C}-5.82421 & 0.79083 & 4.78523 \\
\mathrm{C}-6.27361 & 1.62443 & 3.58632 \\
\mathrm{H}-6.04646 & 1.26106 & 5.74309 \\
\mathrm{H}-6.29307 & -0.19583 & 4.76746 \\
\mathrm{C}-5.41014 & 1.08179 & 2.45859 \\
\mathrm{H}-6.05719 & 2.68135 & 3.75653 \\
\mathrm{H}-7.33603 & 1.52432 & 3.36520 \\
\mathrm{O}-4.19846 & 0.59723 & 3.10069 \\
\mathrm{H}-5.12554 & 1.83169 & 1.72500 \\
\mathrm{H}-5.87783 & 0.23716 & 1.95005 \\
\mathrm{H}-3.87898 & -0.22112 & 4.97119 \\
\mathrm{H}-3.78611 & 1.55083 & 4.89356 \\
\mathrm{H}-1.16024 & -5.98168 & 1.85567 \\
\mathrm{H}-2.73761 & -6.74678 & 1.64809 \\
\mathrm{H}-1.99376 & -6.69139 & 3.24024
\end{array}
$$

Thiophene dimer radical anion meta coordination conformer 2

Electronic energy calculated $(E)=-12502.88616$

Standard state Gibbs free energy $\left(G^{\circ}\right)=-12502.60570$

Number of imaginary frequencies (Nimag) $=0$

$$
\begin{array}{llll}
\text { C }-4.09520 & 1.24850 & -0.23204 \\
\text { C }-3.72177 & -0.01950 & 0.02577 \\
\text { C }-2.33434 & -0.11402 & 0.44516 \\
\text { C }-1.64481 & 1.14458 & 0.30637 \\
\text { S }-2.80417 & 2.43592 & -0.03393 \\
\text { C }-0.27832 & 1.40929 & 0.36351 \\
\text { C } 0.41112 & 2.62238 & 0.24011 \\
\text { C } 1.81397 & 2.52197 & 0.36116 \\
\text { C } 2.20895 & 1.22878 & 0.57738 \\
\text { S } 0.87917 & 0.09726 & 0.62677 \\
\text { Br } 3.97559 & 0.61445 & 0.80860 \\
\text { Br }-5.83556 & 1.86915 & -0.62138
\end{array}
$$




$$
\begin{aligned}
& \text { C } 2.747333 .691930 .26643 \\
& \text { H } 3.452983 .57365-0.55937 \\
& \text { H } 3.336973 .807511 .17894 \\
& \text { H 2.19251 4.61617 } 0.10483 \\
& \text { H - } 0.093443 .565510 .06898 \\
& \text { C -4.62277 - } 1.21487-0.03424 \\
& \mathrm{H}-5.36381-1.11891-0.82818 \\
& \text { H -5.16638 -1.34047 } 0.90672 \\
& \text { H -4.04724 -2.12504 - } 0.20784 \\
& \mathrm{H}-1.79182-1.028600 .20203 \\
& \text { C -1.30312 -4.79200 } 2.99294 \\
& \text { C }-1.37923-3.461982 .45949 \\
& \text { C }-2.15871-2.557793 .13767 \\
& \text { S -2.84102 -3.38666 } 4.50474 \\
& \text { C }-2.06040-4.884114 .11891 \\
& \mathrm{H}-0.83423-3.198001 .55703 \\
& \text { Mg -2.51554 -0.48499 2.71340 } \\
& \text { Br -2.29015 -6.43270 5.18102 } \\
& \text { Br }-4.583690 .538493 .71482 \\
& \text { C }-0.50347-5.905572 .38550 \\
& \text { C }-0.990732 .008023 .80223 \\
& \text { C } 0.449052 .294684 .17759 \\
& \text { C } 0.785141 .103485 .07489 \\
& \text { H } 0.557373 .255784 .68054 \\
& \text { H } 1.076042 .295913 .28301 \\
& \text { C } 0.06782-0.048434 .38701 \\
& \text { H } 0.384101 .259586 .07885 \\
& \text { H } 1.855750 .917615 .16046 \\
& \text { O }-1.051250 .563363 .68655 \\
& \mathrm{H}-0.32816-0.795645 .07215 \\
& \text { H } 0.69237-0.537443 .63975 \\
& \mathrm{H}-1.296162 .429302 .84707 \\
& \text { H - } 1.691252 .317944 .58016 \\
& \text { H } 0.01032-5.564181 .48628 \\
& \text { H - } 1.13734-6.751632 .10835 \\
& \text { H } 0.25134-6.284233 .07929
\end{aligned}
$$

Thiophene dimer radical anion sulfur coordination (13)

Electronic energy calculated $(E)=-12502.89111$

Standard state Gibbs free energy $\left(G^{\circ}\right)=-12502.60910$

Number of imaginary frequencies (Nimag) $=0$

C -4.294252 .267310 .31793$

C -4.047901 .110080 .97692$

C -2.647820 .764610 .97337$

C -1.838401 .654660 .34694$

S -2.82566 2.98338 -0.27334 
C -0.401711 .622270 .15086$

C 0.472452 .666510 .06726

C $1.835292 .28776-0.13204$

C $1.946950 .93314-0.19764$

S $0.434540 .10763-0.03961$

Br $3.53557-0.05279-0.41805$

$\mathrm{Br}-6.328383 .858870 .84515$

C $2.968533 .26415-0.20999$

H $3.715082 .95765-0.94361$

H 3.475273 .353370 .75445

H $2.606814 .25526-0.48476$

H 0.164233 .699740 .17174

C -5.085640 .264721 .65414$

H -6.062700 .742121 .59892$

H -4.839710 .107682 .70776$

H -5.15691 -0.72116 1.18678

H -2.26744 -0.12985 1.45529

H $0.198951 .40952-2.84355$

Br -2.98453 -0.09749 -3.07711

H -5.66314 4.30614 -2.07329

C $0.005112 .10169-3.66018$

Mg -3.00564 2.35093 -2.84038

H - $0.304981 .54256-4.54215$

H $1.758893 .20807-3.05159$

C $-5.417444 .39967-3.12751$

C $1.155273 .05486-3.94846$

H $1.806102 .67188-4.73390$

O -1.11254 2.95644 -3.27154

C $-4.381933 .71195-3.70495$

C $-6.184525 .26170-3.98445$

C $-7.343666 .07570-3.49802$

C $-5.698845 .21221-5.25271$

S $-4.344454 .13887-5.39426$

C $-0.738154 .35614-3.38723$

H $-0.463064 .71944-2.39466$

C $0.441924 .35004-4.33659$

Br -6.34482 6.15268 -6.76275

H $1.065775 .23567-4.21762$

H $0.095524 .30657-5.37153$

H - $1.608614 .90237-3.74459$

H $-7.805536 .65197-4.29921$

H - $7.026786 .77341-2.71893$

H -8.10976 $5.43242-3.05858$

Thiophene dimer $\mathrm{C}-\mathrm{Br}$ abstraction transition state (TS-Thio)

Electronic energy calculated $(E)=-12502.88531$ 
Standard state Gibbs free energy $\left(\mathrm{G}^{\circ}\right)=-12502.60234$

Number of imaginary frequencies $(\mathrm{Nimag})=1$

C $-1.46878-0.478421 .52475$

C $-1.68793-1.536452 .39289$

C $-0.47958-2.121342 .86782$

C $0.66249-1.512032 .42819$

S $0.24047-0.147541 .42685$

C $2.03888-1.859412 .68022$

C $3.14697-1.078872 .51379$

C $4.37547-1.722562 .83140$

C $4.14590-2.992953 .26519$

S $2.47892-3.445413 .24707$

Br $5.44936-4.194083 .88892$

Br -2.627271 .691762 .42011$

C $5.72284-1.089492 .68225$

H $6.41273-1.766262 .17225$

H $6.16804-0.835483 .64691$

H $5.64850-0.175952 .09188$

H $3.09619-0.047972 .18843$

C -3.03095 -2.03794 2.83861

H -3.79785 -1.27555 2.71032

H -3.01239 -2.33184 3.89142

H -3.32921 -2.92089 2.26601

H $-0.46332-2.960283 .55593$

H $2.33710-0.52210-1.33677$

$\mathrm{Br}-0.57201-2.76032-1.81561$

H -3.53579 $1.68532-0.22361$

C $1.99436-0.00369-2.23024$

Mg -0.98605 -0.41551-1.09839

H $1.87674-0.72194-3.04203$

H $3.351371 .61357-1.75331$

C -3.60266 1.42310 -1.27382

C $2.877231 .16909-2.63134$

H $3.661730 .86845-3.32662$

O $0.682570 .57039-1.94906$

C $-2.675230 .61452-1.87695$

C -4.68022 $1.90622-2.09177$

C $-5.772152 .78892-1.57025$

C -4.54756 $1.43685-3.36062$

S -3.15006 $0.42896-3.54591$

C $0.676171 .97732-2.32859$

H $0.781822 .57189-1.41869$

C $1.875122 .14509-3.24282$

Br -5.69125 1.77935-4.82941

H $2.235383 .17463-3.25866$

H $1.627491 .85374-4.26651$ 
H $-0.281982 .19083-2.79583$

H $-6.489793 .05898-2.34543$

H -5.36197 3.71314 -1.15452

H -6.31981 2.29214 -0.76443

Thiophene dimer Mg-ion cage complex (14)

Electronic energy calculated $(E)=-12502.91438$

Standard state Gibbs free energy $\left(G^{\circ}\right)=-12502.63108$

Number of imaginary frequencies (Nimag) $=0$

C $-2.851202 .33155-0.87823$

C -3.370832 .115460 .44881$

C -2.432411 .686151 .36605$

C -1.143771 .550450 .83438$

S - $1.191361 .97483-0.87635$

C 0.035201 .131491 .48369

C 1.307241 .014260 .94369

C 2.290600 .568081 .84592

C 1.737160 .348453 .08507

S 0.048080 .673723 .17098

Br 2.65410 -0.24964 4.60248

$\mathrm{Br}-3.41753$ 5.52736 - 1.12213

C 3.732580 .362791 .50236

H $4.03142-0.675221 .66183$

H 4.378330 .988062 .12206

H 3.917630 .612830 .45858

H $1.525341 .24845-0.09061$

C -4.811662 .343730 .76542$

H -5.100163 .361690 .49642$

H -5.02173 2.18169 1.82293

H -5.444981 .673550 .17885$

H - 2.662231 .474452 .40356

H - 1.112624 .34381 -3.32422

Br -3.47913 0.84334-4.03809

H -6.51438 5.10733 -1.66195

C $-1.561824 .28194-4.31583$

Mg -3.93134 3.12328 -2.65397

H - $1.227743 .36945-4.81167$

H - $1.170266 .39807-4.51101$

C $-6.902154 .28737-2.25933$

C $-1.322305 .53010-5.15605$

H $-0.449175 .42538-5.80013$

O - $2.997004 .18932-4.16061$

C -6.07264 3.33417-2.79124

C $-8.309474 .16014-2.52040$

C $-9.332465 .12258-1.99887$ 


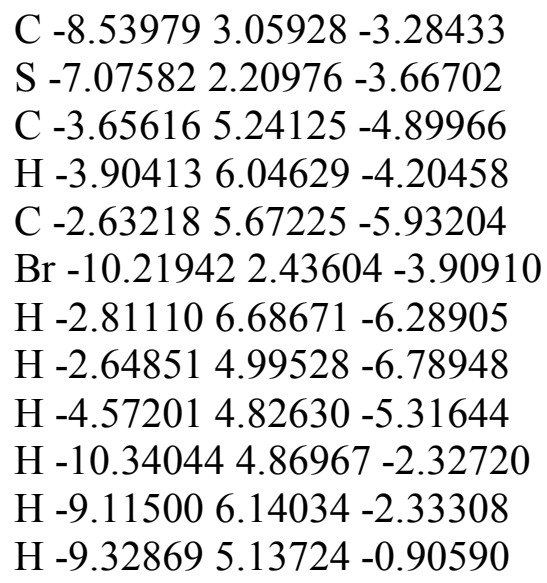

$\underline{\text { Fluorene dimer radical anion monomer coordination }}$

Electronic energy calculated $(E)=-12465.60374$

Standard state Gibbs free energy $\left(G^{\circ}\right)=-12464.88232$

Number of imaginary frequencies (Nimag) $=0$

C $-2.91470-0.766177 .09211$

C -2.743670 .598067 .35418$

C -1.661891 .002458 .14611$

C -0.787960 .060918 .65444$

C - $0.97355-1.298678 .38453$

C - $2.03977-1.716337 .59977$

C 0.434830 .282579 .53242

C $0.91874-1.147509 .72557$

C $0.09236-2.056699 .05065$

C $0.34179-3.424159 .08141$

C $1.44080-3.894759 .80373$

C $2.21205-2.9563910 .44498$

C $2.01836-1.5961910 .45229$

H - 1.527492 .054478 .37455

H -2.19237 -2.76639 7.37414

H $2.67585-0.9127710 .98125$

H $1.66440-4.954809 .84706$

Br 4.313161 .4379512 .04994

H - $0.30046-4.123828 .55648$

C -3.69153 1.597316.80239

C -3.23086 2.83558 6.33847

C -4.100363 .783805 .81924$

C -5.456223 .491475 .76081$

C -5.062391.319516.73616

C -5.933022 .259746 .21953$

C -6.594734 .278575 .27653$

C -7.758513.52058 5.44773

C -7.444562.166306.06654 
C -6.655205 .551484 .72612$

C -7.88797 6.06723 4.34501

C -9.031865 .300614 .52233$

C -8.990324 .024975 .07178$

H -5.43612 0.37317 7.11271

H -2.169083 .051676 .36617$

H -3.71498 4.73252 5.46134

H -5.76010 6.14785 4.58882

H -7.95588 7.05774 3.91368

$\mathrm{Br}-10.719706 .023273 .99314$

H -9.900503 .450965 .19777$

C -8.136742 .000907 .42667$

H -7.868012 .809958 .10830$

H -7.84945 1.054557 .89006

H -9.222751 .999137 .30911$

C -7.843381 .018075 .12914$

$\mathrm{H}-7.552200 .056135 .55669$

H -7.36240 1.11784 4.15436

H -8.92497 1.00211 4.97689

C 0.058220 .9307610 .87208

$\mathrm{H}-0.688050 .3369511 .40364$

H - 0.352301 .9302810 .71156

H 0.938961 .0300611 .50997

C 1.491351 .135988 .81594

H 1.104072 .138878 .62148

H 1.778550 .690437 .86144

H 2.38609 1.235439.43369

H -3.73526 - 1.087976 .46102

H $5.07988-4.1712014 .77392$

H 4.38328 -1.46905 15.06439

C $4.40189-4.0630113 .92426$

H $6.15569-3.4485912 .74419$

C $3.85432-1.7680214 .15707$

O $4.82924-1.8984513 .09068$

C $5.07893-3.2995712 .79886$

H $4.08745-5.0575313 .60753$

H $2.84325-3.3240315 .28974$

C $3.23348-3.1469014 .28756$

H $3.15620-0.9835913 .87174$

Br 7.44527 0.11716 14.47564

H $4.63540-3.5193311 .82616$

Mg $6.04753-0.3756612 .41587$

H 2.41602 -3.26651 13.57280

H $9.08217-0.9766611 .58834$

C $7.10762-1.1652010 .72746$

C $8.50972-1.2947510 .71771$ 
H $11.34992-0.2430510 .50256$

H $11.14529-2.5875911 .49969$

C $6.44770-1.602309 .56053$

C $11.45439-0.695569 .51406$

C $11.25386-3.0010810 .49465$

H $5.36257-1.528959 .50252$

C $9.19214-1.818929 .62955$

C $10.69149-2.026059 .45110$

H $12.52001-0.848179 .32538$

H 11.074580 .012688 .77505

H $12.31681-3.1868310 .32112$

H 10.73021 -3.95863 10.46309

C $7.10718-2.132928 .45506$

C $8.49164-2.239278 .49577$

C $10.74248-2.624888 .05284$

H $6.54750-2.455077 .58096$

C $9.45580-2.742057 .51121$

H $12.85253-2.941927 .73978$

C $11.85203-3.027607 .33255$

C $9.27563-3.266036 .23809$

C $11.65036-3.549796 .06003$

H $8.28535-3.361935 .80640$

C $10.38481-3.674125 .50518$

Br 13.16805 -4.11502 5.04096

H 10.26190 -4.08462 4.51093

$\underline{\text { THF }}$

Electronic energy calculated $(E)=-232.38752$

Standard state Gibbs free energy $\left(G^{\circ}\right)=-232.29381$

Number of imaginary frequencies (Nimag) $=0$

C $3.002260 .41341-3.71869$

C $2.81611-0.44422-2.47303$

O $3.682120 .10920-1.47470$

C $4.571311 .05907-2.07333$

C $4.460100 .84989-3.57774$

H $2.341511 .28370-3.68633$

H $2.79918-0.13550-4.63887$

H $3.10706-1.48375-2.66186$

H $1.79318-0.43663-2.09128$

H $5.576470 .88754-1.68342$

H $4.259012 .07153-1.79237$

H $5.129550 .04906-3.90278$

H $4.701911 .74925-4.14519$

Benzotriazole monomer aggregate 1 
Electronic energy calculated $(E)=-12494.75083$

Standard state Gibbs free energy $\left(G^{\circ}\right)=-12494.10578$

Number of imaginary frequencies (Nimag) $=0$

$\begin{array}{cccc}\mathrm{O} & -2.10091 & 0.22985 & 2.23392 \\ \mathrm{C} & -2.23192 & -0.36535 & 3.55482 \\ \mathrm{C} & -3.66126 & -0.06913 & 3.96884 \\ \mathrm{H} & -3.73313 & 0.92604 & 4.41387 \\ \mathrm{H} & -4.03389 & -0.79593 & 4.69053 \\ \mathrm{C} & -4.40185 & -0.11460 & 2.63265 \\ \mathrm{H} & -5.35581 & 0.41212 & 2.64919 \\ \mathrm{H} & -4.58572 & -1.14880 & 2.33346 \\ \mathrm{H} & -1.47461 & 0.08278 & 4.19406 \\ \mathrm{H} & -2.04363 & -1.43537 & 3.45665 \\ \mathrm{C} & -3.40962 & 0.54127 & 1.68755 \\ \mathrm{H} & -3.51409 & 1.62754 & 1.66814 \\ \mathrm{H} & -3.45445 & 0.15460 & 0.66954 \\ \mathrm{C} & -1.22272 & 2.13676 & -1.21570 \\ \mathrm{C} & -1.48250 & 3.62906 & -1.29083 \\ \mathrm{C} & -0.40130 & 4.18993 & -0.36762 \\ \mathrm{H} & -0.62497 & 5.19209 & -0.00246 \\ \mathrm{C} & -0.36964 & 3.17247 & 0.75951 \\ \mathrm{H} & -1.10013 & 3.39671 & 1.53922 \\ \mathrm{H} & 0.61135 & 3.03712 & 1.21003 \\ \mathrm{H} & 0.56142 & 4.21632 & -0.88200 \\ \mathrm{H} & -2.47789 & 3.86592 & -0.90766 \\ \mathrm{H} & -1.40989 & 4.00147 & -2.31248 \\ \mathrm{O} & -0.75549 & 1.91246 & 0.14273 \\ \mathrm{H} & -2.10544 & 1.51903 & -1.37178 \\ \mathrm{H} & -0.43012 & 1.82571 & -1.89681 \\ \mathrm{Mg} & -0.46729 & 0.08333 & 0.99658 \\ \mathrm{Br} & 1.42145 & 0.26422 & 2.60995 \\ \mathrm{C} & -0.85222 & -1.51002 & -0.37830 \\ \mathrm{O} & 5.87120 & -0.09766 & -1.84867 \\ \mathrm{C} & 6.63789 & 1.13003 & -1.93824 \\ \mathrm{C} & 7.84388 & 0.77468 & -2.78858 \\ \mathrm{H} & 8.63490 & 0.34645 & -2.16872 \\ \mathrm{H} & 8.24654 & 1.64462 & -3.30699 \\ \mathrm{C} & 7.27308 & -0.28072 & -3.73533 \\ \mathrm{H} & 8.03761 & -0.91825 & -4.17896 \\ \mathrm{H} & 6.71103 & 0.19489 & -4.54210 \\ \mathrm{H} & 6.88447 & 1.44272 & -0.92497 \\ \mathrm{H} & 6.3408291017 & 1.88806 & -2.41113 \\ \mathrm{H} & -1.06483 & -2.82841 \\ \mathrm{H} & -1.85964 & -2.28559 \\ \mathrm{H} & -1.47717 & -3.33914 \\ \mathrm{H} & -0.10973 & 2.07674\end{array}$




\begin{tabular}{|c|c|c|c|}
\hline $\mathrm{C}$ & 6.11314 & 0.97762 & 2.94765 \\
\hline $\mathrm{C}$ & 5.07567 & 2.09277 & 2.81324 \\
\hline $\mathrm{H}$ & 5.47327 & 3.07945 & 3.05042 \\
\hline $\mathrm{C}$ & 4.64853 & 1.99267 & 1.35611 \\
\hline $\mathrm{H}$ & 5.24570 & 2.63007 & 0.70229 \\
\hline $\mathrm{H}$ & 3.59159 & 2.19136 & 1.19539 \\
\hline $\mathrm{H}$ & 4.22499 & 1.89737 & 3.46854 \\
\hline $\mathrm{H}$ & 7.08094 & 1.29633 & 2.55285 \\
\hline $\mathrm{H}$ & 6.25305 & 0.64534 & 3.97628 \\
\hline $\mathrm{O}$ & 4.90981 & 0.61211 & 0.97174 \\
\hline $\mathrm{H}$ & 6.23908 & -0.80803 & 1.65552 \\
\hline $\mathrm{H}$ & 4.73356 & -0.66314 & 2.59524 \\
\hline $\mathrm{Mg}$ & 4.21345 & -0.35740 & -0.67955 \\
\hline $\mathrm{Br}$ & 2.55838 & 1.20137 & -1.71258 \\
\hline $\mathrm{C}$ & 3.91748 & -2.45345 & -0.38240 \\
\hline $\mathrm{C}$ & -1.19211 & -2.79102 & 0.13569 \\
\hline $\mathrm{C}$ & -1.42917 & -3.94740 & -0.65048 \\
\hline $\mathrm{C}$ & -1.34025 & -3.85380 & -2.05307 \\
\hline $\mathrm{C}$ & -1.02387 & -2.64128 & -2.58907 \\
\hline $\mathrm{C}$ & -0.78510 & -1.50429 & -1.75663 \\
\hline $\mathrm{H}$ & -0.51644 & -0.59226 & -2.28215 \\
\hline $\mathrm{H}$ & -0.94455 & -2.53245 & -3.66474 \\
\hline $\mathrm{N}$ & -1.32924 & -3.16638 & 1.42804 \\
\hline $\mathrm{N}$ & -1.61428 & -4.44405 & 1.36311 \\
\hline $\mathrm{N}$ & -1.69648 & -4.98135 & 0.16207 \\
\hline $\mathrm{C}$ & -1.89840 & -5.22455 & 2.55432 \\
\hline $\mathrm{H}$ & -1.28290 & -4.84980 & 3.36698 \\
\hline $\mathrm{H}$ & -1.65929 & -6.26276 & 2.34455 \\
\hline $\mathrm{H}$ & -2.95306 & -5.13159 & 2.81210 \\
\hline $\mathrm{Br}$ & -1.65335 & -5.38658 & -3.13759 \\
\hline $\mathrm{C}$ & 2.69927 & -3.09769 & -0.47306 \\
\hline $\mathrm{C}$ & 2.50029 & -4.49740 & -0.26050 \\
\hline $\mathrm{C}$ & 3.54123 & -5.31706 & 0.05743 \\
\hline $\mathrm{C}$ & 4.82015 & -4.73831 & 0.17302 \\
\hline $\mathrm{C}$ & 4.97389 & -3.34476 & -0.04685 \\
\hline $\mathrm{H}$ & 1.80398 & -2.53066 & -0.71204 \\
\hline $\mathrm{H}$ & 1.50053 & -4.90707 & -0.35319 \\
\hline $\mathrm{Br}$ & 3.30546 & -7.18386 & 0.34665 \\
\hline $\mathrm{N}$ & 6.01586 & -5.26983 & 0.46854 \\
\hline $\mathrm{N}$ & 6.82377 & -4.23075 & 0.41773 \\
\hline $\mathrm{N}$ & 6.28750 & -3.06956 & 0.12715 \\
\hline $\mathrm{C}$ & 8.23169 & -4.36072 & 0.74776 \\
\hline $\mathrm{H}$ & 8.54932 & -5.36709 & 0.49162 \\
\hline $\mathrm{H}$ & 8.37739 & -4.18570 & 1.81344 \\
\hline $\mathrm{H}$ & 8.79055 & -3.62832 & 0.17242 \\
\hline
\end{tabular}

Benzotriazole monomer aggregate 2 
Electronic energy calculated $(E)=-12494.75202$

Standard state Gibbs free energy $\left(G^{\circ}\right)=-12494.10618$

Number of imaginary frequencies (Nimag) $=0$

\begin{tabular}{|c|c|c|c|}
\hline $\mathrm{O}$ & 1.62123 & 2.93930 & 0.38277 \\
\hline $\mathrm{C}$ & 0.19345 & 3.18667 & 0.23178 \\
\hline $\mathrm{C}$ & -0.08767 & 4.39599 & 1.10579 \\
\hline $\mathrm{H}$ & -0.29938 & 4.08507 & 2.13126 \\
\hline $\mathrm{H}$ & -0.93819 & 4.97016 & 0.73891 \\
\hline $\mathrm{C}$ & 1.23205 & 5.16403 & 1.04278 \\
\hline $\mathrm{H}$ & 1.36113 & 5.86469 & 1.86759 \\
\hline $\mathrm{H}$ & 1.30938 & 5.71767 & 0.10407 \\
\hline $\mathrm{H}$ & -0.33574 & 2.28457 & 0.53030 \\
\hline $\mathrm{H}$ & -0.00019 & 3.38899 & -0.82299 \\
\hline $\mathrm{C}$ & 2.25654 & 4.04678 & 1.08560 \\
\hline $\mathrm{H}$ & 2.46318 & 3.71947 & 2.10592 \\
\hline $\mathrm{H}$ & 3.19034 & 4.26896 & 0.57248 \\
\hline $\mathrm{C}$ & 4.52008 & -4.01911 & 1.32013 \\
\hline $\mathrm{C}$ & 3.66479 & -4.69372 & 2.37735 \\
\hline $\mathrm{C}$ & 2.42291 & -3.80467 & 2.39677 \\
\hline $\mathrm{H}$ & 1.84406 & -3.89143 & 3.31631 \\
\hline $\mathrm{C}$ & 3.01699 & -2.41728 & 2.23042 \\
\hline $\mathrm{H}$ & 3.32914 & -1.99287 & 3.18708 \\
\hline $\mathrm{H}$ & 2.36886 & -1.71371 & 1.71392 \\
\hline $\mathrm{H}$ & 1.76791 & -4.04402 & 1.55554 \\
\hline $\mathrm{H}$ & 4.16836 & -4.66901 & 3.34658 \\
\hline $\mathrm{H}$ & 3.45075 & -5.73290 & 2.12729 \\
\hline $\mathrm{O}$ & 4.20251 & -2.60732 & 1.41331 \\
\hline $\mathrm{H}$ & 5.59081 & -4.14245 & 1.47822 \\
\hline $\mathrm{H}$ & 4.26227 & -4.35920 & 0.31448 \\
\hline $\mathrm{Mg}$ & 2.65050 & 1.40855 & -0.47714 \\
\hline $\mathrm{Br}$ & 0.98804 & -0.43474 & -0.75409 \\
\hline $\mathrm{C}$ & 3.74852 & 1.95934 & -2.22963 \\
\hline $\mathrm{O}$ & 5.97381 & -2.04168 & -0.67202 \\
\hline $\mathrm{C}$ & 4.89924 & -2.07727 & -1.65226 \\
\hline $\mathrm{C}$ & 5.46333 & -2.83567 & -2.84749 \\
\hline $\mathrm{H}$ & 5.87802 & -2.14089 & -3.58086 \\
\hline $\mathrm{H}$ & 4.69855 & -3.43339 & -3.34239 \\
\hline $\mathrm{C}$ & 6.57981 & -3.67865 & -2.22924 \\
\hline $\mathrm{H}$ & 7.33623 & -3.98170 & -2.95303 \\
\hline $\mathrm{H}$ & 6.17380 & -4.57728 & -1.75896 \\
\hline $\mathrm{H}$ & 4.61837 & -1.05147 & -1.88904 \\
\hline $\mathrm{H}$ & 4.05141 & -2.57811 & -1.18751 \\
\hline $\mathrm{C}$ & 7.13952 & -2.74028 & -1.17948 \\
\hline $\mathrm{H}$ & 7.82235 & -2.00755 & -1.61562 \\
\hline $\mathrm{H}$ & 7.62257 & -3.22884 & -0.33525 \\
\hline $\mathrm{C}$ & 8.24376 & 0.74792 & 1.47228 \\
\hline
\end{tabular}




$\begin{array}{cccc}\mathrm{C} & 8.97699 & 1.77879 & 0.63209 \\ \mathrm{C} & 7.91796 & 2.16437 & -0.39881 \\ \mathrm{H} & 8.33164 & 2.61916 & -1.29914 \\ \mathrm{C} & 7.25525 & 0.83009 & -0.68900 \\ \mathrm{H} & 7.79172 & 0.26901 & -1.45682 \\ \mathrm{H} & 6.20760 & 0.91759 & -0.97045 \\ \mathrm{H} & 7.19283 & 2.85611 & 0.03501 \\ \mathrm{H} & 9.84336 & 1.32843 & 0.14168 \\ \mathrm{H} & 9.32252 & 2.62055 & 1.23228 \\ \mathrm{O} & 7.33640 & 0.08546 & 0.55294 \\ \mathrm{H} & 8.88546 & -0.01101 & 1.91423 \\ \mathrm{H} & 7.64977 & 1.21520 & 2.26024 \\ \mathrm{Mg} & 5.83020 & -1.24171 & 1.23223 \\ \mathrm{Br} & 4.21910 & 0.84981 & 1.52463 \\ \mathrm{C} & 6.80674 & -1.84964 & 3.05962 \\ \mathrm{C} & 7.76110 & -2.89977 & 3.13856 \\ \mathrm{C} & 8.39957 & -3.33828 & 4.32746 \\ \mathrm{C} & 8.08875 & -2.70526 & 5.54659 \\ \mathrm{C} & 7.17616 & -1.69321 & 5.51989 \\ \mathrm{C} & 6.55755 & -1.28757 & 4.29665 \\ \mathrm{H} & 5.84538 & -0.46981 & 4.38603 \\ \mathrm{H} & 6.91141 & -1.18312 & 6.43935 \\ \mathrm{Br} & 8.92868 & -3.26756 & 7.16103 \\ \mathrm{~N} & 8.23861 & -3.67517 & 2.13657 \\ \mathrm{~N} & 9.08246 & -4.48559 & 2.72989 \\ \mathrm{~N} & 9.23317 & -4.34683 & 4.03074 \\ \mathrm{C} & 9.82676 & -5.49599 & 1.99882 \\ \mathrm{H} & 9.54370 & -5.42777 & 0.95349 \\ \mathrm{H} & 9.58016 & -6.48003 & 2.39276 \\ \mathrm{H} & 10.89305 & -5.31097 & 2.11338 \\ \mathrm{C} & 4.61598 & 3.08377 & -2.29885 \\ \mathrm{C} & 5.38981 & 3.44206 & -3.43246 \\ \mathrm{C} & 5.31335 & 2.65218 & -4.59680 \\ \mathrm{C} & 4.48248 & 1.57257 & -4.57854 \\ \mathrm{C} & 3.72260 & 1.24688 & -3.41203 \\ \mathrm{H} & 4.39252 & 0.94599 & -5.45854 \\ \mathrm{~N} & 6.09636 & 4.54660 & -3.14897 \\ \mathrm{~N} & 5.74592 & 4.81263 & -1.90673 \\ \mathrm{H} & 4.88084 & 4.00422 & -1.34378 \\ \mathrm{H} & 7.23940 & 5.99684 & -1.22642 \\ \mathrm{H} & 5.56297 & 6.22892 & -1.61802 \\ \mathrm{H} & 5.78733 & -0.16196 \\ \mathrm{H} & 3.10660 & -1.40658 \\ \mathrm{H} & 0.36725 & -3.49720\end{array}$




\section{References}

(1) Woods, E. F.; Berl, A. J.; Kalow, J. A. Photocontrolled Synthesis of n-Type Conjugated Polymers. Angew. Chemie Int. Ed. 2020.

(2) Neese, F. The ORCA Program System. WIREs Comput. Mol. Sci. 2012, 2, 73-78.

(3) Neese, F. Software Update: The ORCA Program System, Version 4.0. WIREs Comput. Mol. Sci. 2018, 8, e1327.

(4) Hanwell, M. D.; Curtis, D. E.; Lonie, D. C.; Vandermeersch, T.; Zurek, E.; Hutchison, G. R. Avogadro: An Advanced Semantic Chemical Editor, Visualization, and Analysis Platform. J. Cheminform. 2012, 4, 17.

(5) Yanai, T.; Tew, D. P.; Handy, N. C. A New Hybrid Exchange-Correlation Functional Using the Coulomb-Attenuating Method (CAM-B3LYP). Chem. Phys. Lett. 2004, 393, 51-57.

(6) Barone, V.; Cossi, M. Quantum Calculation of Molecular Energies and Energy Gradients in Solution by a Conductor Solvent Model. J. Phys. Chem. A 1998, 102, 1995-2001.

(7) Grimme, S. Supramolecular Binding Thermodynamics by Dispersion-Corrected Density Functional Theory. Chem. - A Eur. J. 2012, 18, 9955-9964.

(8) Treitel, N.; Shenhar, R.; Aprahamian, I.; Sheradsky, T.; Rabinovitz, M. Calculations of PAH Anions: When Are Diffuse Functions Necessary? Phys. Chem. Chem. Phys. 2004, 6, 1113-1121.

(9) Maji, M. S.; Pfeifer, T.; Studer, A. Oxidative Homocoupling of Aryl, Alkenyl, and Alkynyl Grignard Reagents with TEMPO and Dioxygen. Angew. Chemie Int. Ed. 2008, 47, 9547-9550.

(10) Bhattacharjee, H. R.; Patel, G. N. Quantum Yield of Solid State Polymerization of Diacetylenes. J. Photochem. 1981, 16, 85-91.

(11) Michaudel, Q.; Chauviré, T.; Kottisch, V.; Supej, M. J.; Stawiasz, K. J.; Shen, L.; Zipfel, W. R.; Abruña, H. D.; Freed, J. H.; Fors, B. P. Mechanistic Insight into the Photocontrolled Cationic Polymerization of Vinyl Ethers. J. Am. Chem. Soc. 2017, 139, 15530-15538.

(12) Kuchel, P. W.; Vaughan, R. J. Average Lengths of Chords in a Square. Math. Mag. 1981, 54, 261-269.

(13) Stoll, S.; Schweiger, A. EasySpin, a Comprehensive Software Package for Spectral Simulation and Analysis in EPR. J. Magn. Reson. 2006, 178, 42-55.

(14) Weil, J. A.; Bolton, J. R. Electron Paramagnetic Resonance: Elementary Theory and Practical Applications, Second Edition, 2nd Editio.; John Wiley \& Sons, Ltd: Hoboken, NJ, 2006. 